\title{
On $\boldsymbol{n}$-dependent groups and fields II
}

\author{
Artem Chernikov ${ }^{1}$ and Nadja Hempel ${ }^{2}$ \\ ${ }^{1}$ Department of Mathematics, University of California Los Angeles, Los Angeles, CA \\ 90095-1555, USA; E-mail: chernikov@ math.ucla.edu. \\ ${ }^{2}$ Mathematisches Institut, Albert-Ludwigs-Universität Freiburg, Ernst-Zermelo-Str. 1, \\ 79104 Freiburg, Deutschland; E-mail: hempel@math.uni-freiburg.de.
}

Received: 12 February 2020; Revised: 10 February 2021; Accepted: 21 March 2021

2020 Mathematics Subject Classification: 03C45; 03C60

\begin{abstract}
We continue the study of $n$-dependent groups, fields and related structures, largely motivated by the conjecture that every $n$-dependent field is dependent. We provide evidence toward this conjecture by showing that every infinite $n$ dependent valued field of positive characteristic is henselian, obtaining a variant of Shelah's Henselianity Conjecture in this case and generalizing a recent result of Johnson for dependent fields. Additionally, we prove a result on intersections of type-definable connected components over generic sets of parameters in $n$-dependent groups, generalizing Shelah's absoluteness of $G^{00}$ in dependent theories and relative absoluteness of $G^{00}$ in 2-dependent theories. In an effort to clarify the scope of this conjecture, we provide new examples of strictly 2-dependent fields with additional structure, showing that Granger's examples of non-degenerate bilinear forms over dependent fields are 2-dependent. Along the way, we obtain some purely model-theoretic results of independent interest: we show that $n$-dependence is witnessed by formulas with all but one variable singletons; provide a type-counting criterion for 2-dependence and use it to deduce 2-dependence for compositions of dependent relations with arbitrary binary functions (the Composition Lemma); and show that an expansion of a geometric theory $T$ by a generic predicate is dependent if and only if it is $n$-dependent for some $n$, if and only if the algebraic closure in $T$ is disintegrated. An appendix by Martin Bays provides an explicit isomorphism in the Kaplan-Scanlon-Wagner theorem.
\end{abstract}

\section{Introduction}

A classical line of research in model theory, both pure and applied, aims to determine properties of algebraic structures, such as groups and fields, that satisfy certain model-theoretic tameness assumptions. This is analogous to the study of algebraic or Lie groups in algebraic or differential geometry, but instead of considering groups definable in a specific structure like $\mathbb{C}$ or $\mathbb{R}$, one typically considers groups definable in a class of first-order structures with some restrictions on the complexity of their definable subsets. Some of the most striking applications of model theory are based on a detailed understanding of definable groups in certain specific contexts of this kind (e.g. Hrushovski's proof of the Mordell-Lang conjecture for function fields [24] is based on the theory of stable groups, applied to groups definable in differentially closed and in separably closed fields). But even if one is only interested in abstract classification of first-order structures, the study of definable groups unavoidably enters the picture (e.g. through Zilber's work on totally categorical structures [47] or Hrushovski's theorem on unidimensional theories [23]). In the case of model-theoretically tame fields, one often expects not only to deduce some of their general properties, but in fact to obtain an explicit algebraic classification. Probably the first result of this type is Macintyre's proof that all $\boldsymbol{\aleph}_{0}$-stable fields (roughly speaking, fields admitting a Zariski-like notion of dimension on their definable subsets) are algebraically closed 
[33], generalized by Cherlin-Shelah to the larger class of superstable fields [9]. Some of the longeststanding conjectures in model theory that played a fundamental role in the development of the area are asking for characterizations of this type, such as Podewski's conjecture whether all minimal fields are algebraically closed [37], or the stable field conjecture asking if all stable fields are separably closed (see e.g. [29]), and we discuss some further examples below.

In this article, we continue the study of groups, fields and related structures satisfying a modeltheoretic tameness condition called $n$-dependence, for $n \in \mathbb{N}$, initiated in [22] and continued in [11]. The class of $n$-dependent theories was introduced by Shelah in [40], with the 1-dependent (or just dependent) case corresponding to the class of NIP theories that has attracted a lot of attention recently (see e.g. [42] for an introduction to the area). Basic properties of $n$-dependent theories are investigated in [14]. Roughly speaking, $n$-dependence of a theory guarantees that the edge relation of an infinite generic $(n+1)$-hypergraph is not definable in its models (see Definition 2.1). For $n \geq 2$, we say that a theory is strictly $n$-dependent if it is $n$-dependent but not $(n-1)$-dependent.

This paper is largely, but not exclusively, motivated by the following conjecture.

\section{Conjecture 1.1. There are no strictly $n$-dependent fields for $n \geq 2$ (in the pure ring language).}

We expect that the same should hold for fields expanded with some natural operators such as, for example, derivations or valuations. We also expect a generalization to type-definable fields: every field type-definable in an $n$-dependent structure is isomorphic to a field type-definable in a dependent structure. Some initial evidence toward Conjecture 1.1 is given by the results in [22]: every infinite $n$-dependent field is Artin-Schreier closed (generalizing [31] for $n=1$ ); every non-separable PAC (i.e. pseudo-algebraically closed) field is not $n$-dependent for any $n$ (generalizing [19]). In particular, for fields with (super-)simple theories, our Conjecture 1.1 follows from the well-known conjecture that all such fields are (bounded) PAC (see e.g. [36]). On the other hand, combined with Shelah's conjectures on dependent fields discussed below, Conjecture 1.1 leads to a complete classification of $n$-dependent fields.

In this paper, we obtain some new results about $n$-dependent groups and fields, in part providing further evidence for Conjecture 1.1 and clarifying its scope, and in part generalizing the known results about dependent or 2-dependent structures. First, we prove Shelah's Henselianity Conjecture for $n$ dependent valued fields of positive characteristic in Section 3 (this generalizes Johnson [27] for $n=1$ and, toward Conjecture 1.1, demonstrates that a known property of dependent fields also holds for all $n$-dependent fields). Additionally, we establish a result on intersections of type-definable connected components over generic sets of parameters in $n$-dependent groups in Section 4, generalizing Shelah's theorems on absoluteness of $G^{00}$ in dependent theories and relative absoluteness of $G^{00}$ for 2-dependent theories. While we do not have any direct application of this result toward Conjecture 1.1 at the moment, the $n=1$ case is a fundamental property of dependent groups and is used extensively in Johnson's classification of dependent fields of finite dp-rank [27, 28], so we expect it to be useful in the future study of Conjecture 1.1 and its aforementioned generalization to type-definable fields in $n$-dependent structures. Second, we provide new examples of strictly 2-dependent fields with additional structure by showing that Granger's examples of non-degenerate bilinear forms over dependent fields are strictly 2dependent in Section 6 (demonstrating in particular the necessity of the pure ring language assumption in Conjecture 1.1). Our proof of this relies on establishing some general results on $n$-dependent theories, possibly of independent interest: a reduction of the $n$-dependence of a theory to formulas with all but one of its variables singletons (Section 2), a type-counting criterion for 2-dependence and the Composition Lemma showing 2-dependence of compositions of dependent relations with binary functions (Section 5). And third, we show in Section 7 that an expansion of a geometric theory $T$ by a generic predicate is dependent if and only if it is $n$-dependent for some $n$, if and only if the algebraic closure in $T$ is disintegrated (generalizing the $n=1$ case from [6]). While not directly related to Conjecture 1.1, this gives an example of a class of structures (expansions of geometric structures by a generic predicate) for which $n$-dependence is equivalent to dependence, the behavior predicted for fields by Conjecture 1.1, and the authors hope that some of the arguments might be useful in the future for the original question. 
In the rest of the introduction, we discuss these results in further detail and overview the structure of the paper.

One of the results in [14] gives a characterization of $n$-dependence in terms of generalized indiscernibles (indexed by ordered random partite $n$-hypergraphs) and demonstrates, using this characterization, that in order to verify $n$-dependence of a theory it is enough to check that every formula $\varphi\left(x ; y_{1}, \ldots, y_{n}\right)$ with at least one of the tuples of variables $x, y_{1}, \ldots, y_{n}$ singleton is $n$-dependent (generalizing the well-known theorem of Shelah for dependent theories). In Section 2, we refine and generalize some of these results allowing indexing structures of larger cardinalities and obtaining a better reduction to singletons: a theory $T$ is $n$-dependent if and only if every formula $\varphi\left(x, y_{1}, \ldots, y_{n}\right)$ such that all but at most one of the tuples $x, y_{1}, \ldots, y_{n}$ are singletons is $n$-dependent (Theorem 2.12).

In Section 3 (which is self-contained except for the results in Appendix A), we obtain further evidence toward Conjecture 1.1 in the case of valued fields. The question of classifying dependent (valued) fields is currently an active area of research motivated by various versions of Shelah's Conjecture, which in particular predicts that every infinite dependent valued field is henselian. A recent result of Johnson $[27,28]$ confirms this for valued fields of positive characteristic. In Theorem 3.1, we generalize this by showing that every $n$-dependent valued field of positive characteristic is henselian, for arbitrary $n$. As in Johnson's proof, the theorem is deduced by showing that any two valuations on an infinite $n$-dependent field of positive characteristic must be comparable. In the case of $n=1$, this lemma can be quickly obtained using Artin-Schreier closedness of dependent fields and absoluteness of the connected component $G^{00}$ (in fact, an application of Baldwin-Saxl is sufficient). However, replacing the absolute connected component with a weaker condition for intersections of uniformly definable families of subgroups available in $n$-dependent theories (Proposition 3.9) requires a detailed analysis of the effect that the isomorphism for special linear groups from Kaplan-Scanlon-Wagner [31] has on multiple valuations. We are able to carry it out, relying in particular on the explicit description of this isomorphism given by Bays in Appendix A. Concerning the (open) case of characteristic 0, in Section 3.5 we observe that the model completions of multi-ordered and multi-valued fields with at least two orders (respectively, valuations) as studied in $[45,30]$ are not $n$-dependent for any $n$.

Given a definable group $G$ and a small set of parameters $A$ (see Section 2.1 for the definitions of 'small', 'saturated', etc.), we denote by $G_{A}^{00}$ the intersection of all subgroups of $G$ of bounded index typedefinable over $A$ (see Section 4.1 for more details). A crucial fact about definable groups in dependent theories, due to Shelah, is that for every small set $A$, one has $G_{A}^{00}=G_{\emptyset}^{00}$ [39]. This can be viewed as an infinitary analog of the Baldwin-Saxl condition on intersections of uniformly definable families of subgroups in dependent theories [2]. In [41], Shelah established the following result for groups definable in 2-dependent theories: let $\mathcal{M}$ be a sufficiently saturated model, and let $b$ be a finite tuple in $\mathbb{M}$ (and not contained in $\mathcal{M}$ in the case of interest); then $G_{\mathcal{M} \cup b}^{00}=G_{\mathcal{M}}^{00} \cap G_{C b}^{00}$ for some small set $C \subseteq \mathcal{M}$. In Section 4 (which is self-contained), we generalize this result from 2-dependent groups to $n$-dependent groups. Specifically, we show that if $T$ is $n$-dependent and $G=G(\mathbb{M})$ is a type-definable group (over $\emptyset$ ), then for any small model $M$ and finite tuples $b_{1}, \ldots, b_{n-1}$ sufficiently independent over $M$ in an appropriate sense, we have that

$$
G_{\mathcal{M} \cup b_{1} \cup \ldots \cup b_{n-1}}^{00}=\bigcap_{i=1, \ldots, n-1} G_{\mathcal{M} \cup b_{1} \cup \ldots \cup b_{i-1} \cup b_{i+1} \cup \ldots \cup b_{n-1}}^{00} \cap G_{C \cup b_{1} \cup \ldots \cup b_{n-1}}^{00}
$$

for some $C \subseteq \mathcal{M}$ of absolutely bounded size (Theorem 4.9 and Corollary 4.10). In other words, the intersection of all subgroups of $G$ of bounded index type-definable over $\mathcal{M} \cup b_{1} \cup \cdots \cup b_{n-1}$ is already given by the intersection of a (potentially) smaller collection of subgroups containing only boundedly many groups whose definitions involve all $n-1$ of the parameters $b_{1}, \ldots, b_{n-1}$ at the same time. Our independence assumption on the parameters holds trivially in the cases $n=1,2$ giving the aforementioned results for dependent and 2-dependent groups, and in general can be achieved assuming that the $b_{i}$ 's appear as the vertices of an amalgamation diagram with respect to the independence relation of being a $\kappa$-coheir (see Definition 4.8 for the precise definition of our independence assumption). While 
this result has no direct applications to Conjecture 1.1 at the moment (in our proof of Theorem 3.1 we only needed a chain condition for uniformly definable families of subgroups from Proposition 3.9), we expect that it will be useful in the future, in particular for the aforementioned variant of Conjecture 1.1 for type-definable fields.

Next, we consider the limitations of Conjecture 1.1 (in terms of the additional structure allowed on the field) and try to place it in a more general model-theoretic setting. In [22], it was observed that the theory of a bilinear form on an infinite dimensional vector space over a finite field is strictly 2-dependent. In fact, all of the previously known 'algebraic' examples of strictly $n$-dependent theories with $n \geq 2$ tend to look like multi-linear forms over finite fields. For example, smoothly approximable structures are 2-dependent and coordinatizable via bilinear forms over finite fields [8]; and the strictly $n$-dependent pure groups constructed in [11] using Mekler's construction are essentially of this form as well, using Baudisch's interpretation of Mekler's construction in alternating bilinear maps [3]. In Section 6, we show that one can replace finite fields by arbitrary dependent fields in these examples. Namely, we investigate $n$-dependence for theories of bilinear forms on vector spaces with a separate sort for the field, in the sense of Granger [21]. We show that all such theories are 2-dependent assuming that the field is dependent, and that the assumption of dependence is necessary (see Theorem 6.3). Combined with the fact that the intersection conditions on the connected components discussed above resemble modular behavior in the 2-dependent case, this leads one to speculate that $n$-dependence of a theory might imply some form of 'linearity relative to the dependent part'. While formulating this precisely appears difficult at the moment, we view Conjecture 1.1 as a specific instance of this general principle. Our proof of Theorem 6.3 relies on the criterion for $n$-dependence in terms of generalized indiscernibles from Section 2, and on some additional purely model-theoretic results contained in Section 5 that we now describe.

In [14], a generalization of the Sauer-Shelah lemma to $n$-dependent formulas is given, in particular demonstrating that a formula $\varphi\left(x ; y_{1}, \ldots, y_{n}\right)$ is $n$-dependent if and only if the number of $\varphi$-types over an arbitrary large finite set $A$ of parameters is bounded by $2^{|A|^{n-\varepsilon}}$ for some $\varepsilon=\varepsilon(\varphi) \in \mathbb{R}_{>0}$. Concerning the number of types over infinite sets of parameters, a well-known theorem of Shelah [38, Theorem II.4.11] shows that if $\varphi(x, y)$ is dependent, then the number of $\varphi$-types over an infinite set of parameters of size $\kappa$ is at $\operatorname{most} \operatorname{ded}(\kappa)$, where $\operatorname{ded}(\kappa)$ is the supremum over the number of Dedekind cuts in a linear order of cardinality $\kappa$. In Section 5.1, we show that a theory is 2-dependent if and only if the following type-counting criterion is satisfied. Let $c$ be a finite tuple and $I$ an indiscernible sequence of size $\kappa$. Then the number of types over $I c$ that are realized cofinally in a sequence mutually indiscernible to $I$ is bounded by $\operatorname{ded}(\kappa)$ (see Proposition 5.6 for details). In Section 5.2, this criterion is combined with set-theoretic absoluteness to obtain a more general version of the following finitary combinatorial statement of independent interest, the 'Composition Lemma'. Let $R \subseteq M^{3}$ be a ternary relation definable in a dependent structure, and let $f: M^{2} \rightarrow M$ be an arbitrary (not necessarily definable) function. Then the ternary relation $R^{\prime}(x, y, z)=R(f(x, y), f(x, z), f(y, z))$ is 2-dependent (Theorem 5.12). It is interesting to compare this to a line of results around Hilbert's 13th problem demonstrating that a function of arbitrary arity can be expressed as a finite composition of binary functions (in the category of all functions, or of continuous functions on $\mathbb{R}$ - a celebrated theorem of Kolmogorov and Arnold [1]). Our result can be viewed as saying that in such presentations, the outer relation is necessarily 'fractal-like'. It is worth mentioning that some other connections of $n$-dependence to finitary hypergraph combinatorics are considered in [44] (in connection to hypergraph growth) and in [17] (which establishes a strong regularity lemma for $n$-dependent hypergraphs demonstrating that every $n$-dependent relation of arbitrarily high arity can be approximated by relations of arity $n$ up to measure 0). The Composition Lemma is applied in the proof of Theorem 6.3 to conclude 2-dependence of certain basic atomic formulas involving the 'generic' binary function given by the bilinear form.

Finally, in Section 7 (which only depends on Section 2), we consider $n$-dependence for expansions of geometric theories by generic predicates and relations of higher arity. In particular, we show that an expansion of a geometric theory $T$ by a generic predicate is dependent if and only if it is $n$-dependent for some $n$, if and only if the algebraic closure in $T$ is disintegrated (Corollary 7.13). This generalizes the corresponding result for dependence in [6]. In Remark 7.8, we give an example showing that 
geometricity of $T$ (or some other additional assumption) is necessary even for $n=1$. Our proof for relations of higher arity relies on an infinitary generalization of Hrushovski's observation [25] that the random $n$-ary hypergraph is not a finite Boolean combination of relations of arity $n-1$.

\section{Preliminaries and some general lemmas on $n$-dependence}

\subsection{Notation}

We will be following standard model-theoretic notation; refer to, for example, [43, 34] for an introduction to model theory. Usually $T$ will denote a complete first-order theory in a language $\mathcal{L}$ (possibly multisorted), and $\mathcal{M}, \mathcal{N}$ will be first-order $\mathcal{L}$-structures. We recall that, given an infinite cardinal $\kappa$, a first-order structure $\mathcal{M}$ is $\kappa$-saturated when for every set of formulas with parameters from a subset of $\mathcal{M}$ of size $<\kappa$ in a fixed finite tuple of variables for which every finite subset of these formulas is satisfied by a tuple in $\mathcal{M}$, the whole set is satisfied by a tuple in $\mathcal{M}$. Given a tuple $a$ of elements in $\mathcal{M}$ and a subset $B$ of $\mathcal{M}$, we denote by $\operatorname{tp}(a / B)$ the complete type of $a$ over $b$ (i.e. the collection of all formulas with parameters in $B$ satisfied by $a)$. We write $a \equiv_{B} a^{\prime}$ when $\operatorname{tp}(a / B)=\operatorname{tp}\left(a^{\prime} / B\right)$. A structure $\mathcal{M}$ is $\kappa$-homogeneous if for any two finite tuples $a, a^{\prime}$ and a set of parameters $B$ in $\mathcal{M}$ with $|B|<\kappa$ for which $a \equiv_{B} a^{\prime}$, there exists an automorphism $\sigma \in \operatorname{Aut}(\mathcal{M} / B)$ of $\mathcal{M}$ fixing $B$ pointwise and sending $a$ to $a^{\prime}$. We let $\mathbb{M} \vDash T$ be a monster model of $T$ : that is, a $\kappa$-saturated and $\kappa$-homogeneous model of $T$ for some sufficiently large strongly inaccessible cardinal $\kappa=\kappa(\mathbb{M})$. Once $\mathbb{M}$ is fixed, as usual we will say that a subset of $\mathbb{M}$ (or just an arbitrary set/structure) is small if it has cardinality smaller than $\kappa(\mathbb{M})$. Given an $\mathcal{L}$-structure $\mathcal{M} \models T$ and a single variable (of a prescribed sort of the language $\mathcal{L}$ ), we write $\mathcal{M}_{x}$ to denote the corresponding sort of $\mathcal{M}$. If $x=\left(x_{1}, \ldots, x_{n}\right)$ is a finite tuple of variables, we let $\mathcal{M}_{x}:=\prod_{i=1}^{n} \mathcal{M}_{x_{i}}$.

\subsection{N-dependent formulas and their basic properties}

We begin with the definition of $n$-dependent theories and some of the basic properties of $n$-dependent formulas and theories.

Definition 2.1. A partitioned formula $\varphi\left(x ; y_{1}, \ldots, y_{n}\right)$ has the $n$-independence property (with respect to a theory $T$ ) if, in some model of $T$, there is a sequence of tuples $\left(a_{1, i}, \ldots, a_{n, i}\right)_{i \in \omega}$ such that for every $s \subseteq \omega^{n}$, there is a tuple $b_{s}$ with the following property:

$$
\vDash \varphi\left(b_{s} ; a_{1, i_{1}}, \ldots, a_{n, i_{n}}\right) \Leftrightarrow\left(i_{1}, \ldots, i_{n}\right) \in s .
$$

Otherwise, we say that $\varphi\left(x, y_{1}, \ldots, y_{n}\right)$ is $n$-dependent. A theory is $n$-dependent if it implies that every formula is $n$-dependent.

We simply say that a theory is dependent if it is 1-dependent. A structure $\mathcal{M}$ is $n$-dependent if $\operatorname{Th}(\mathcal{M})$ is $n$-dependent.

\section{Fact 2.2 [14, Proposition 6.5].}

1. Let $\varphi\left(x, y_{1}, \ldots, y_{n}\right)$ and $\psi\left(x, y_{1}, \ldots, y_{n}\right)$ be $n$-dependent formulas. Then $\neg \varphi, \varphi \wedge \psi$ and $\varphi \vee \psi$ are $n$-dependent.

2. Let $\varphi\left(x, y_{1}, \ldots, y_{n}\right)$ be a formula. Suppose that $\left(w, z_{1}, \ldots, z_{n}\right)$ is any permutation of the tuple $\left(x, y_{1}, \ldots, y_{n}\right)$. Then $\psi\left(w, z_{1}, \ldots, z_{n}\right):=\varphi\left(x, y_{1}, \ldots, y_{n}\right)$ is $n$-dependent if and only if $\varphi\left(x, y_{1}, \ldots, y_{n}\right)$ is n-dependent.

3. A theory $T$ is $n$-dependent if and only if every formula $\varphi\left(x, y_{1}, \ldots, y_{n}\right)$ with $|x|=1$ is $n$-dependent (see also Section 2.4).

\subsection{Generalized indiscernibles}

We will often use a characterization of $n$-dependence from [14] in terms of generalized indiscernibles.

Definition 2.3. Fix a language $\mathcal{L}_{\text {opg }}^{n}=\left\{R_{n}\left(x_{1}, \ldots, x_{n}\right),<, P_{1}(x), \ldots, P_{n}(x)\right\}$. An ordered n-partite hypergraph is an $\mathcal{L}_{\text {opg }}^{n}$-structure $\mathcal{A}=\left(A ;<, R_{n}, P_{1}, \ldots, P_{n}\right)$ such that: 
1. $A$ is the disjoint union $P_{1}^{\mathcal{A}} \sqcup \ldots \sqcup P_{n}^{\mathcal{A}}$.

2. $R_{n}^{\mathcal{A}}$ is a symmetric relation such that if $\left(a_{1}, \ldots, a_{n}\right) \in R_{n}^{\mathcal{A}}$ then $P_{i}^{\mathcal{A}} \cap\left\{a_{1}, \ldots, a_{n}\right\}$ is a singleton for every $1 \leq i \leq n$.

3. $<^{\mathcal{A}}$ is a linear ordering on $A$ with $P_{1}^{\mathcal{A}}<\ldots<P_{n}^{\mathcal{A}}$.

\section{Fact 2.4.}

1. [14, Proposition A.5] Let $\mathcal{K}$ be the class of all finite ordered n-partite hypergraphs. Then $\mathcal{K}$ is a Fraïssé class, and its limit is called the generic ordered $n$-partite hypergraph, denoted by $G_{n, p}$.

2. [14, Remark 4.5] An ordered n-partite hypergraph $\mathcal{A}$ is a model of $\operatorname{Th}\left(G_{n, p}\right)$ if and only if:

$\circ\left(P_{i}^{\mathcal{A}},<\right)$ is a dense linear order without endpoints for each $1 \leq i \leq n$.

$\circ$ For every $1 \leq j \leq n$, finite disjoint sets $A_{0}, A_{1} \subset \prod_{1 \leq i \leq n, i \neq j} P_{i}^{\mathcal{A}}$ and $b_{0}<b_{1} \in P_{j}^{\mathcal{A}}$, there is some $b \in P_{j}^{\mathcal{A}}$ such that $b_{0}<b<b_{1}$ and: $R_{n}(b, \bar{a})$ holds for every $\bar{a} \in A_{0}$ and $\neg R_{n}(b, \bar{a})$ holds for every $\bar{a} \in A_{1}$.

We denote by $O_{n, p}$ the reduct of $G_{n, p}$ to the language $\mathcal{L}_{\text {op }}^{n}=\left\{<, P_{1}(x), \ldots, P_{n}(x)\right\}$.

Remark 2.5. It is easy to see from the axiomatization in Fact 2.4(2) that given $G_{n, p}$ and any non-empty intervals $I_{t} \subseteq P_{t}$ for $t=1, \ldots, n$, the set $I_{1} \times \ldots \times I_{n}$ contains an induced copy of $G_{n, p}$.

Definition 2.6. Let $T$ be a theory in a language $\mathcal{L}$, and let $\mathbb{M}$ be a monster model of $T$.

1. Let $I$ be a structure in the language $\mathcal{L}_{0}$. We say that $\bar{a}=\left(a_{i}\right)_{i \in I}$, with $a_{i}$ a tuple in $\mathbb{M}$, is $I$-indiscernible over a set of parameters $C \subseteq \mathbb{M}$ if for all $n \in \omega$ and all $i_{0}, \ldots, i_{n}$ and $j_{0}, \ldots, j_{n}$ from $I$, we have

$$
\begin{aligned}
& \operatorname{qftp}_{\mathcal{L}_{0}}\left(i_{0}, \ldots, i_{n}\right)=\operatorname{qftp}_{\mathcal{L}_{0}}\left(j_{0}, \ldots, j_{n}\right) \Rightarrow \\
& \operatorname{tp}_{\mathcal{L}}\left(a_{i_{0}}, \ldots, a_{i_{n}} / C\right)=\operatorname{tp}_{\mathcal{L}}\left(a_{j_{0}}, \ldots, a_{j_{n}} / C\right) .
\end{aligned}
$$

2. For $\mathcal{L}_{0}$-structures $I$ and $J$, we say that $\left(b_{i}\right)_{i \in J}$ is based on $\left(a_{i}\right)_{i \in I}$ over a set of parameters $C \subseteq \mathbb{M}$ if for any finite set $\Delta$ of $\mathcal{L}(C)$-formulas, and for any finite tuple $\left(j_{0}, \ldots, j_{n}\right)$ from $J$, there is a tuple $\left(i_{0}, \ldots, i_{n}\right)$ from $I$ such that:

$\circ \operatorname{qftp}_{\mathcal{L}_{0}}\left(j_{0}, \ldots, j_{n}\right)=\operatorname{qftp}_{\mathcal{L}_{0}}\left(i_{0}, \ldots, i_{n}\right)$ and

$\circ \operatorname{tp}_{\Delta}\left(b_{j_{0}}, \ldots, b_{j_{n}}\right)=\operatorname{tp}_{\Delta}\left(a_{i_{0}}, \ldots, a_{i_{n}}\right)$.

The following general fact is used to find $G_{n, p}$-indiscernibles.

Fact 2.7 [14, Corollary 4.8]. Let $C \subseteq \mathbb{M}$ be a small set of parameters.

1. For any $n \in \omega$ and $\bar{a}=\left(a_{g}\right)_{g \in O_{n, p}}$, there is some $\left(b_{g}\right)_{g \in O_{n, p}}$ that is $O_{n, p}$-indiscernible over $C$ and based on à over $C$.

2. For any $n \in \omega$ and $\bar{a}=\left(a_{g}\right)_{g \in G_{n, p}}$, there is some $\left(b_{g}\right)_{g \in G_{n, p}}$ that is $G_{n, p}$-indiscernible over $C$ and based on ā over $C$.

Using this, we can characterize $n$-dependence of a formula as follows.

Proposition 2.8. The following are equivalent, in any theory $T$.

1. $\varphi\left(x ; y_{1}, \ldots, y_{n}\right)$ is not $n$-dependent.

2. There are tuples $b$ and $\left(a_{g}\right)_{g \in G_{n, p}}$ such that

(a) $\left(a_{g}\right)_{g \in G_{n, p}}$ is $O_{n, p}$-indiscernible over $\emptyset$ and $G_{n, p}$-indiscernible over $b$;

(b) $\vDash \varphi\left(b ; a_{g_{1}}, \ldots, a_{g_{n}}\right) \Longleftrightarrow G_{n, p} \vDash R_{n}\left(g_{1}, \ldots, g_{n}\right)$, for all $g_{i} \in P_{i}$.

3. (2) holds for any small $G_{n, p}^{\prime} \equiv G_{n, p}$ in the place of $G_{n, p}$.

Proof. The equivalence of (1) and (2) is the equivalence of (a) and (c) in [14, Lemma 6.2], and (3) implies (2) is obvious. Given a witness to (2), we can find a witness to (3) by compactness as every finite substructure of $G_{n, p}^{\prime}$ appears as a finite substructure of $G_{n, p}$. 
Additionally, we have the following 'formula-free' characterization of $n$-dependence of a theory.

Proposition 2.9. Let $T$ be a complete theory, and let $\mathbb{M}=T$ be a monster model. Then for any $n \in \mathbb{N}$, the following are equivalent:

1. $T$ is n-dependent.

2. For any $\left(a_{g}\right)_{g \in G_{n, p}}$ and $b$ with $a_{g}$, b finite tuples in $\mathbb{M}$, if $\left(a_{g}\right)_{g \in G_{n, p}}$ is $G_{n, p}$-indiscernible over $b$ and $O_{n, p}$-indiscernible (over $\emptyset$ ), then it is $O_{n, p}$-indiscernible over $b$.

3. For any small $G_{n, p}^{\prime} \equiv G_{n, p},\left(a_{g}\right)_{g \in G_{n, p}^{\prime}}$ and $b$, if $\left(a_{g}\right)_{g \in G_{n, p}^{\prime}}$ is $G_{n, p}^{\prime}$-indiscernible over $b$ and $O_{n, p}^{\prime}$-indiscernible, then it is $O_{n, p}^{\prime}$-indiscernible over b (where $O_{n, p}^{\prime}$ is the $\mathcal{L}_{o p}^{n}$-reduct of $G_{n, p}^{\prime}$ ).

Proof. The equivalence of (1) and (2) is [14, Proposition 6.3], (3) implies (2) is obvious, and we show that (2) implies (3). Assume that (3) fails: that is, there exist some $G_{n, p}^{\prime} \equiv G_{n, p},\left(a_{g}\right)_{g \in G_{n, p}^{\prime}}$ and $b$ small tuples such that $\left(a_{g}\right)_{g \in G_{n, p}^{\prime}}$ is $O_{n, p}^{\prime}$-indiscernible, $G_{n, p}^{\prime}$-indiscernible over $b$, but not $O_{n, p}^{\prime}$-indiscernible over $b$. By definition, this is witnessed by some finite set of formulas and some finite set of indices from $G_{n, p}^{\prime}$. Restricting all of the $a_{g}$ 's and $b$ to the corresponding subtuples appearing in those formulas, we may assume that (3) fails with all of $b$ and $a_{g}$ finite. Moreover, we can choose a countable elementary submodel of $G_{n, p}^{\prime}$ containing all of the indices witnessing failure of indiscernibility. It is isomorphic to $G_{n, p}$ by $\boldsymbol{\aleph}_{0}$-categoricity of $\operatorname{Th}\left(G_{n, p}\right)$, hence restricting $\left(a_{g}\right)_{g \in G_{n, p}^{\prime}}$ to the corresponding set of indices we get a failure of (2).

\subsection{Improved reduction to singletons}

In this section, we will improve Fact 2.2(3) by showing that $T$ is $n$-dependent if and only if every formula in which all but at most one of its variables are singletons is $n$-dependent (as opposed to 'at least one of the variables is a singleton' as in Fact 2.2(3)).

To do so, we need some auxiliary results. First we refine the equivalence of (1) and (2) in Proposition 2.9, making explicit the correspondence between the variables of a formula that is not $n$-dependent and the sorts of the tuples in a generalized indiscernible witnessing it. In the proof below, we are following the proof in [14, Proposition 6.3] with some modifications.

Proposition 2.10. Fix $n \geq 1$, and let $x, y_{1}, \ldots, y_{n-1}$ be some fixed finite tuples of variables. The following are equivalent:

1. There exists some $\left(a_{g}\right)_{g \in G_{n, p}}$ with $a_{g} \in \mathbb{M}_{y_{i}}$ for all $g \in P_{i}, 1 \leq i \leq n-1$ and $a_{g} \in \mathbb{M}_{y_{n}^{\prime}}$ for some finite tuple of variables $y_{n}^{\prime}$ and all $g \in P_{n}$, and $b \in \mathbb{M}_{x}$ such that $\left(a_{g}\right)_{g \in G_{n, p}}$ is $G_{n, p}$-indiscernible over $b$ and $O_{n, p}$-indiscernible over $\emptyset$ but is not $O_{n, p}$-indiscernible over $b$.

2. There exists some formula $\varphi\left(x, y_{1}, \ldots, y_{n-1}, y_{n}^{\prime \prime}\right)$ that is not $n$-dependent, with $y_{n}^{\prime \prime}$ some finite tuple of variables.

Proof. We obtain immediately that (2) implies (1) by the implication (1) $\Rightarrow$ (2) in Proposition 2.8, with $y_{n}^{\prime}=y_{n}^{\prime \prime}$.

To prove (1) implies (2), let $\left(a_{g}\right)_{g \in G_{n, p}}$ and $b$ be as given by (1). We define $a_{g}^{\prime}:=a_{g}$ for all $g \in \bigcup_{1 \leq i \leq n-1} P_{i}$ and $a_{g}^{\prime}:=a_{g} b$ for all $g \in P_{n}$. We have that $\left(a_{g}^{\prime}\right)_{g \in G_{n, p}}$ is not $O_{n, p}$-indiscernible but is $G_{n, p}$-indiscernible (over $\emptyset$ ), from the corresponding properties of $\left(a_{g}\right)_{g \in G_{n, p}}$ over $b$. Namely, by assumption, there are some finite subsets $V, W \subseteq G_{n, p}$ with the $\mathcal{L}_{\mathrm{op}}$-isomorphic induced structures such that $\left(a_{g}\right)_{g \in V} \not_{b}\left(a_{g}\right)_{g \in W}$. Then taking some $h \in P_{n}$ above all of the elements of $V \cup W$ with respect to the order on $P_{n}$, we have that $V h \cong \mathcal{L}_{\text {op }} W h$. However, since the tuple $a_{h}^{\prime}$ contains $b$, we have $\left(a_{g}^{\prime}\right)_{g \in V h} \not \equiv\left(a_{g}^{\prime}\right)_{g \in W h}$.

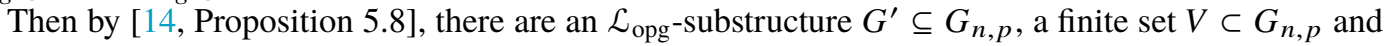
a formula $\psi\left(y_{1}, \ldots, y_{n-1}, \tilde{y}_{n}, z\right) \in \mathcal{L}$ such that $\tilde{y}_{n}=y_{n}^{\prime} x, z$ is a finite tuple of variables corresponding to a fixed enumeration $\left(a_{g}\right)_{g \in V}$ of $V$, and 
1. $G^{\prime} \cong \mathcal{L}_{\text {opg }} G_{n, p}$.

2. $G_{n, p} \vDash R_{n}\left(g_{1}, \ldots, g_{n}\right)$ if and only if $\vDash \psi\left(a_{g_{1}}^{\prime}, \ldots, a_{g_{n}}^{\prime},\left(a_{g}^{\prime}\right)_{g \in V}\right)$, for every $g_{i} \in P_{i}\left(G^{\prime}\right)$.

3. For every finite $W, W^{\prime} \subseteq G^{\prime}$, we have $W V \cong{ }^{\mathcal{L}_{\text {op }}} W^{\prime} V$ whenever $W \cong \mathcal{L}_{\text {op }} W^{\prime}$.

Let $\varphi\left(x, y_{1}, \ldots, y_{n-1}, y_{n}^{\prime \prime}\right)$ be the formula $\psi\left(y_{1}, \ldots, y_{n-1}, y_{n}^{\prime} x, z\right)$ with $y_{n}^{\prime \prime}:=y_{n}^{\prime} z$, and let $a_{g}^{\prime \prime}:=a_{g}^{\prime}=a_{g}$ for $g \in P_{i}\left(G^{\prime}\right), 1 \leq i \leq n-1$, and let $a_{g}^{\prime \prime}:=a_{g}\left(a_{h}\right)_{h \in V}$ for $g \in P_{n}\left(G^{\prime}\right)$. Then $\left(a_{g}^{\prime \prime}\right)_{g \in G^{\prime}}$ is $\mathcal{L}_{\mathrm{op}^{-}}$ indiscernible (by $\mathcal{L}_{\mathrm{op}}$-indiscernibility of $\left(a_{g}^{\prime}\right)_{g \in G_{n, p}}$ and the choice of $V$ ), and $\varphi\left(b, a_{g_{1}}^{\prime \prime}, \ldots, a_{g_{n}}^{\prime \prime}\right.$ ) holds if and only if $R_{n}\left(g_{1}, \ldots, g_{n}\right)$ does, for all $g_{i} \in P_{i}\left(G^{\prime}\right)$. Then $\varphi$ is not $n$-dependent by $(2) \Rightarrow(1)$ in Proposition 2.8.

Lemma 2.11. Let $y_{1}, \ldots, y_{n-1}$ be some fixed finite tuples of variables. If the condition (1) in Proposition 2.10 holds for some finite tuple of variables $x$, then it already holds with $x$ a single variable.

Proof. We assume that (1) fails for $|x|=1$ and prove that then it fails for any tuple of variables $x$ by induction on $|x|$. So let $b \in \mathbb{M}_{x}$ with $|b|>1$ be given, say $b=b_{1} b_{2}$ for some tuples $1 \leq\left|b_{1}\right|,\left|b_{2}\right|<n$. And assume that $\left(a_{g}\right)_{g \in G_{n, p}}$ with $a_{g} \in \mathbb{M}_{y_{i}}$ for $g \in P_{i}, 1 \leq i \leq n-1$ is such that $\left(a_{g}\right)_{g \in G_{n, p}}$ is $G_{n, p^{-}}$-indiscernible over $b$ and $O_{n, p}$-indiscernible over $\emptyset$. We need to show that $\left(a_{g}\right)_{g \in G_{n, p}}$ is $O_{n, p^{-}}$ indiscernible over $b$.

In particular, $\left(a_{g}\right)_{g \in G_{n, p}}$ is $G_{n, p}$-indiscernible over $b_{2}$; hence it is also $O_{n, p}$-indiscernible over $b_{2}$ by the inductive assumption. Let $a_{g}^{\prime}:=a_{g}$ for $g \in P_{i}, 1 \leq i \leq n-1$, and let $a_{g}^{\prime}:=a_{g} b_{2}$ for $g \in P_{n}$. Note that $\left(a_{g}^{\prime}\right)_{g \in G_{n, p}}$ is $G_{n, p}$-indiscernible over $b_{1}$, and is $O_{n, p}$-indiscernible over $\emptyset$ by the previous sentence. Applying the inductive assumption again, we conclude that $\left(a_{g}^{\prime}\right)_{g \in G_{n, p}}$ is $O_{n, p}$-indiscernible over $b_{1}$; hence $\left(a_{g}\right)_{g \in G_{n, p}}$ is $O_{n, p}$-indiscernible over $b=b_{1} b_{2}$.

Using this, we can finally strengthen Fact 2.2(3).

\section{Theorem 2.12.}

1. Assume that the formula $\varphi\left(x, y_{1}, \ldots, y_{n}\right)$ is not $n$-dependent. Then there is a formula $\varphi^{\prime}\left(x^{\prime}, y_{1}, \ldots, y_{n-1}, y_{n}^{\prime}\right)$ that is not $n$-dependent, and such that $x^{\prime}$ is a single variable and $y_{n}^{\prime}$ is some finite tuple of variables extending $y_{n}$.

2. A theory $T$ is $n$-dependent if and only if every formula $\varphi\left(x, y_{1}, \ldots, y_{n}\right)$ such that all but at most one of the tuples $x, y_{1}, \ldots, y_{n}$ are singletons is $n$-dependent.

Proof.

(1) By Lemma 2.11 and the equivalence of (1) and (2) in Proposition 2.10.

(2) Assume that some formula $\varphi\left(x, y_{1}, \ldots, y_{n}\right)$ is not $n$-dependent. Applying (1), we find some formula $\varphi^{\prime}\left(x^{\prime}, y_{1}, \ldots, y_{n-1}, y_{n}^{1}\right)$ that is not $n$-dependent, $x^{\prime}$ is a singleton, and $y_{n}^{1}$ is a tuple of variables extending $y_{n}$. Exchanging the roles of $x^{\prime}$ and $y_{1}$ by Fact 2.2, we thus obtain a formula $\varphi_{1}\left(y_{1}, y_{1}^{\prime}, y_{2}, \ldots, y_{n-1}, y_{n}^{1}\right)$ that is not $n$-dependent and $\left|y_{1}^{\prime}\right|=1$. Repeating the same procedure recursively with $y_{i}$ in the role of $y_{1}$, for $1 \leq i \leq n-1$, we find formulas

$$
\varphi_{i}\left(y_{i}, y_{1}^{\prime}, \ldots, y_{i}^{\prime}, y_{i+1}, \ldots, y_{n-1}, y_{n}^{i}\right)
$$

that are not $n$-dependent, $\left|y_{j}^{\prime}\right|=1$ for $1 \leq j \leq i$ and $y_{n}^{j+1}$ extending $y_{n}^{j}$. Finally, taking $\varphi_{n-1}\left(y_{n-1}, y_{1}^{\prime}, \ldots, y_{n-1}^{\prime}, y_{n}^{n-1}\right)$ and applying (1) one more time, we obtain the desired formula with all but the last variable singletons.

\section{3. $N$-dependent valued fields}

The main result of this section is the following theorem generalizing a recent result of Johnson [27] from $n=1$ to all $n \in \mathbb{N}$.

Theorem 3.1. If $(K, \mathcal{O})$ is an infinite valued field of positive characteristic and $\operatorname{Th}(K)$ is $n$-dependent for some $n \in \mathbb{N}$, then $K$ is henselian. 
From now on, let $K$ be an infinite field of characteristic $p>0$ and $\mathcal{O}_{i}$ a valuation $\operatorname{ring}$ on $K$ for $i=1,2$. We additionally fix the following notation:

○ For $i=1,2$, let $\mathfrak{m}_{i}$ be the maximal ideal of $\mathcal{O}_{i}$.

○ Let $J:=\mathfrak{m}_{1} \cap \mathfrak{m}_{2}$.

Fact 3.2 [27, Remark 2.1]. Assume $\mathcal{O}_{1}$ and $\mathcal{O}_{2}$ are incomparable (i.e. none of them is contained in the other). Then

$$
\left(a+\mathfrak{m}_{1}\right) \cap\left(b+\mathfrak{m}_{2}\right) \neq \emptyset
$$

for any $a \in \mathcal{O}_{1}$ and $b \in \mathcal{O}_{2}$.

Definition 3.3. We say that $b \in K$ is an Artin-Schreier root of $a \in K$ if $a=b^{p}-b$. We call $K$ Artin-Schreier closed if every element of $K$ has an Artin-Schreier root in $K$.

Recall the following.

Fact 3.4 ([31] for $n=1$, [22] for arbitrary $n \in \mathbb{N})$. Let $K$ be an infinite field of positive characteristic, such that $\operatorname{Th}(K)$ is n-dependent. Then $K$ is Artin-Schreier closed.

Our main contribution is the following result.

Proposition 3.5. Suppose that $\left(K, \mathcal{O}_{1}, \mathcal{O}_{2}\right)$ is n-dependent and $\operatorname{ch} \operatorname{ar}(K)=p>0$. Then every element in $J$ has an Artin-Schreier root in $J$.

Being able to find an Artin-Schreier root in both maximal ideals simultaneously forces the corresponding valuations to be comparable:

Corollary 3.6. If the structure $\left(K, \mathcal{O}_{1}, \mathcal{O}_{2}\right)$ is n-dependent and $\operatorname{char}(K)=p>0$, then $\mathcal{O}_{1}$ and $\mathcal{O}_{2}$ are comparable.

Proof. Assume not; then by Fact 3.2 with $a=0$ and $b=1$, there exists some $w \in \mathfrak{m}_{1} \cap\left(1+\mathfrak{m}_{2}\right)$. Let $y:=w^{p}-w$. Now, as $\operatorname{val}_{1}(w)>0$, we have that

$$
\operatorname{val}_{1}(y)=\operatorname{val}_{1}(w)>0 .
$$

Secondly, let $z \in \mathrm{m}_{2}-$ that is, $\operatorname{val}_{2}(z)>0-$ be such that $w=1+z$. Then

$$
\operatorname{val}_{2}(y)=\operatorname{val}_{2}\left(w^{p}-w\right)=\operatorname{val}_{2}\left((1+z)^{p}-(1+z)\right)=\operatorname{val}_{2}\left(z^{p}-z\right)=\operatorname{val}_{2}(z)>0 .
$$

Thus $y \in J$. However, the Artin-Schreier roots of $y$ are exactly $w, w+1, \ldots, w+p-1$, none of which can lie in $\mathfrak{m}_{1} \cap \mathfrak{m}_{2}=J$. This contradicts Proposition 3.5.

Then Theorem 3.1 follows from Corollary 3.6 exactly as in the proof of [27, Theorem 2.8] using that $n$-dependence is preserved under interpretations. Our proof of Proposition 3.5 is given in Section 3.4, but before presenting it we have to develop the following three main ingredients:

- A chain condition for intersections in uniformly definable families of subgroups in $n$-dependent theories, discussed in Section 3.1

- An explicit version of the isomorphism for special linear groups from Kaplan-Scanlon-Wagner [31] (see Section 3.2 for a discussion and Appendix A by Martin Bays for the proofs)

- A detailed analysis of what happens to the valuations of certain elements in the field when this special isomorphism is applied (carried out in Section 3.3)

\subsection{A 'chain condition' for intersections of definable subgroups in n-dependent theories}

Recall the 'chain condition' for definable families of subgroups in $n$-dependent theories. 
Fact 3.7 [22, Proposition 4.1]. Let $G$ be a definable group, and let $\psi\left(x ; y_{0}, \ldots, y_{n-1}\right)$ be an $n$-dependent formula such that $\psi\left(G ; b_{0}, \ldots, b_{n-1}\right)$ is a subgroup of $G$ for any parameters $b_{0}, \ldots, b_{n-1}$. Then there exists some $m_{\psi} \in \omega$ such that for any $d \geq m_{\psi}$ and any array of parameters $\left(b_{i, j}: i<n, j \leq d\right)$, there is some $v \in d^{n}$ such that

$$
\bigcap_{\eta \in d^{n}} H_{\eta}=\bigcap_{\eta \in d^{n}, \eta \neq v} H_{\eta}
$$

where $H_{\eta}:=\psi\left(G ; b_{0, i_{0}}, \ldots, b_{n-1, i_{n-1}}\right)$ for $\eta=\left(i_{0}, \ldots, i_{n-1}\right)$.

We generalize it to simultaneous intersections of several definable families of subgroups. Before we do so, let us recall the partite version of Ramsey's theorem.

\section{Fact 3.8.}

1. (Infinitary version) For every $m, n \in \omega$ and any function $f: \omega^{n} \rightarrow m$, there exist some infinite sets $s_{0}, \ldots, s_{n-1} \subseteq \omega$ such that $f \uparrow_{s_{0} \times \ldots \times s_{n-1}}$ is constant.

2. (Finitary version) For every $l, m, n \in \omega$, there is some $R=R(l, m, n) \in \omega$ such that for any function $f: R^{n} \rightarrow m$, there are some sets $s_{0}, \ldots, s_{n-1} \subseteq R$ with $\left|s_{0}\right|, \ldots,\left|s_{n-1}\right| \geq l$ and such that $f \uparrow_{s_{0} \times \ldots \times s_{n-1}}$ is constant.

Proposition 3.9. Let $G$ be a definable group, and for $t<k$ let $\psi_{t}\left(x ; y_{0}, \ldots, y_{n-1}\right)$ be an $n$-dependent formula such that $\psi_{t}\left(G ; b_{0}, \ldots, b_{n-1}\right)$ is a subgroup of $G$ for any $t<k$ and any parameters $b_{0}, \ldots, b_{n-1}$. Then there exists some $m=m\left(\psi_{0}, \ldots, \psi_{k-1}\right) \in \omega$ such that for any $d \geq m$ and any array of parameters $\left(b_{i, j}: i<n, j \leq d\right)$, there is a single $v \in d^{n}$ such that for all $t<k$,

$$
\bigcap_{\eta \in d^{n}} H_{\eta}^{t}=\bigcap_{\eta \in d^{n}, \eta \neq v} H_{\eta}^{t}
$$

where $H_{\eta}^{t}:=\psi_{t}\left(G ; b_{0, i_{0}}, \ldots, b_{n-1, i_{n-1}}\right)$ for $\eta=\left(i_{0}, \ldots, i_{n-1}\right)$.

Proof. We argue by induction on $k$, the base case $k=1$ given by Fact 3.7. Let $m_{1}:=m\left(\psi_{0}\right)$ and $m_{2}:=m\left(\psi_{1}, \ldots, \psi_{k-1}\right)$ be given by the inductive hypothesis. Let $R:=R\left(m_{2}, m_{1}^{n}, n\right)$ be given by Fact 3.8(2). We take $m=m\left(\psi_{0}, \ldots, \psi_{k-1}\right):=R m_{1}$.

Let an array $B=\left(b_{i, j}: i<n, j \leq m\right)$ be given. For each $\gamma=\left(\gamma_{0}, \ldots, \gamma_{n-1}\right) \in R^{n}$, consider the subarray

$$
B_{\gamma}=\left(b_{0, \gamma_{0} m_{1}+\eta_{0}}, \ldots, b_{n-1, \gamma_{n-1} m_{1}+\eta_{n-1}}: \eta=\left(\eta_{0}, \ldots, \eta_{n-1}\right) \in m_{1}^{n}\right) .
$$

By the choice of $m_{1}$, for each $\gamma \in R^{n}$, there is some $v_{\gamma} \in m_{1}^{n}$ such that

$$
\bigcap_{\eta \in m_{1}^{n}} H_{\left(\gamma_{0} m_{1}+\eta_{0}, \ldots, \gamma_{n-1} m_{1}+\eta_{n-1}\right)}^{0}=\bigcap_{\eta \in m_{1}^{n}, \eta \neq \gamma_{\gamma}} H_{\left(\gamma_{0} m_{1}+\eta_{0}, \ldots, \gamma_{n-1} m_{1}+\eta_{n-1}\right)}^{0} .
$$

By the choice of $R$, there are some sets $s_{0}, \ldots, s_{n-1} \subseteq R$ with $\left|s_{0}\right|=\ldots=\left|s_{n-1}\right|=m_{2}$ such that $v_{\gamma}$ is equal to some fixed $v^{\prime}=\left(v_{0}^{\prime}, \ldots, v_{n-1}^{\prime}\right) \in m_{1}^{n}$, for all $\gamma \in s_{0} \times \ldots \times s_{n-1}$. Consider the array

$$
B^{\prime}=\left(b_{0, \gamma_{0} m_{1}+v_{0}^{\prime}}, \ldots, b_{n-1, \gamma_{n-1} m_{1}+v_{n-1}^{\prime}}: \gamma \in s_{1} \times \ldots \times s_{n-1}\right) .
$$

By the choice of $m_{2}$, there is some $\gamma^{\prime} \in s_{1} \times \ldots \times s_{n-1}$ such that

$$
\bigcap_{\gamma \in s_{0} \times \ldots \times s_{n-1}} H_{\left(\gamma_{0} m_{1}+v_{0}^{\prime}, \ldots, \gamma_{n-1} m_{1}+v_{n-1}^{\prime}\right)}^{t}=
$$

$\bigcap_{\gamma \in s_{0} \times \ldots \times s_{n-1}, \gamma \neq \gamma^{\prime}} H_{\left(\gamma_{0} m_{1}+\gamma_{0}^{\prime}, \ldots, \gamma_{n-1} m_{1}+\gamma_{n-1}^{\prime}\right)}^{t}$ for all $1 \leq t<k$. 
Let $v:=\left(\gamma_{0}^{\prime} m_{1}+v_{0}^{\prime}, \ldots, \gamma_{n-1}^{\prime} m_{1}+v_{n-1}^{\prime}\right)$. By $(*)$ and $(* *)$, we have

$$
\bigcap_{\eta \in m^{n}} H_{\eta}^{t}=\bigcap_{\eta \in m^{n}, \eta \neq v} H_{\eta}^{t} \text { for all } 0 \leq t<k,
$$

as desired.

\subsection{Special vector groups and their explicit isomorphisms}

Let $K$ be a field of characteristic $p>0$. We let $\mathbb{K}$ be the algebraic closure of $K, \mathcal{K}$ a perfect subfield of $K$, and let $\wp(x)$ be the additive homomorphism $x \mapsto x^{p}-x$ on $\mathbb{K}$. We consider the following algebraic subgroups of $(\mathbb{K},+)^{n}$ :

Definition 3.10. For a singleton $a$ in $\mathbb{K}$, we let $G_{a}$ be equal to $(\mathbb{K},+)$, and for a tuple $\bar{a}=\left(a_{0}, \ldots, a_{n-1}\right) \in$ $\mathbb{K}^{n}$ with $n>1$, we define:

$$
G_{\bar{a}}=\left\{\left(x_{0}, \ldots, x_{n-1}\right) \in \mathbb{K}^{n}: a_{0} \cdot \wp\left(x_{0}\right)=a_{i} \cdot \wp\left(x_{i}\right) \text { for } 0 \leq i<n\right\} .
$$

Recall that for an algebraic group $G$, we denote by $G^{0}$ the connected component of the unit element of $G$ (in the Zariski topology). Note that if $G$ is definable over some parameter set $A$, its connected component $G^{0}$ coincides with the smallest $A$-definable subgroup of $G$ of finite index (in $\mathbb{K}$ ). We have the following sufficient condition for connectedness of $G_{\bar{a}}$.

Fact 3.11 [22, Lemma 5.3]. Let $\bar{a}=\left(a_{0}, \ldots, a_{n-1}\right)$ be a tuple in $\mathbb{K}^{\times}$for which the set $\left\{\frac{1}{a_{0}}, \ldots, \frac{1}{a_{n-1}}\right\}$ is linearly $\mathbb{F}_{p}$-independent. Then $G_{\bar{a}}$ is connected.

Moreover, under the same assumption on $\bar{a}$, these groups are isomorphic to the additive group of the field:

Fact 3.12 [22, Corollary 5.4]. Let $\mathcal{K}$ be a perfect subfield of an algebraically closed field $\mathbb{K}$, and let $\bar{a} \in \mathcal{K}^{n}$ be such that the set $\left\{\frac{1}{a_{0}}, \ldots, \frac{1}{a_{n-1}}\right\}$ is linearly $\mathbb{F}_{p}$-independent. Then $G_{\bar{a}}$ is (algebraically) isomorphic to $(\mathbb{K},+)$ over $\mathcal{K}$. In particular, for any field $K$ with $\mathcal{K} \leq K \leq \mathbb{K}$, the group $G_{\bar{a}}(K)$ is isomorphic to $(K,+)$.

In Appendix A, Bays provides an explicit description of such an isomorphism that we now describe to set up the notation.

Given arbitrary $m \in \mathbb{N}$ and $x_{1}, \ldots, x_{m} \in K$, the corresponding Moore matrix is the $m \times m$ matrix

$$
M\left(x_{1}, \ldots, x_{m}\right)=\left(\begin{array}{ccc}
x_{1} & \ldots & x_{m} \\
x_{1}^{p} & \ldots & x_{m}^{p} \\
\vdots & & \vdots \\
x_{1}^{p^{m-1}} & \ldots & x_{m}^{p^{m-1}}
\end{array}\right) \text {, }
$$

and the Moore determinant is $\Delta\left(x_{1}, \ldots, x_{m}\right):=\operatorname{det} M\left(x_{1}, \ldots, x_{m}\right)$. By Fact A.1, the set $\left\{x_{1}, \ldots, x_{m}\right\}$ is linearly independent over $\mathbb{F}_{p}$ if and only if $\Delta\left(x_{1}, \ldots, x_{m}\right) \neq 0$.

Now, fix $\bar{a}=\left(a_{0}, \ldots, a_{m}\right) \in \mathcal{K}^{m+1}$ such that the set $\left\{\frac{1}{a_{0}}, \ldots, \frac{1}{a_{m}}\right\}$ is $\mathbb{F}_{p}$-linearly independent, and let

$$
A:=M\left(a_{0}^{-\frac{1}{p^{m}}}, \ldots, a_{m}^{-\frac{1}{p^{m}}}\right) \text {. }
$$

Note that $\left\{a_{0}^{-\frac{1}{p^{m}}}, \ldots, a_{m}^{-\frac{1}{p^{m}}}\right\}$ is still an $\mathbb{F}_{p}$-linearly independent subset of $\mathcal{K}$; hence $A$ is invertible by 
Fact A.1. We define

$$
\bar{\alpha}=\left(\begin{array}{c}
\alpha_{0} \\
\vdots \\
\alpha_{m-1} \\
\alpha_{m}
\end{array}\right):=A^{-1}\left(\begin{array}{c}
0 \\
\vdots \\
0 \\
1
\end{array}\right)
$$

that is, $\alpha_{i}=\left(A^{-1}\right)_{i, m} \in \mathcal{K}$ for $0 \leq i \leq m$. One still has that $\left(\alpha_{0}, \ldots, \alpha_{m}\right)$ are linearly $\mathbb{F}_{p}$-independent (see Claim A.2); hence $M\left(\alpha_{0}, \ldots, \alpha_{m}\right)$ is invertible. Let $\beta_{i, j} \in \mathcal{K}$ be the entries of the inverse matrix of $M\left(\alpha_{0}, \ldots, \alpha_{m}\right)$. Then we have:

Fact 3.13. The map $f_{\bar{a}}: G_{\bar{a}}(\mathbb{K}) \rightarrow(\mathbb{K},+)$ given by

$$
f_{\bar{a}}\left(x_{0}, \ldots, x_{m}\right):=\sum_{j=0}^{m} \alpha_{j} x_{j}
$$

is a group isomorphism, and $f_{\bar{a}}^{-1}:(\mathbb{K},+) \rightarrow G_{\bar{a}}(\mathbb{K})$ given by

$$
f_{\bar{a}}^{-1}(t):=\left(\sum_{j=0}^{m} \beta_{i, j} t^{p^{j}}: 0 \leq i \leq m\right)
$$

is its inverse.

\subsection{The effect of the isomorphism $f_{\bar{a}}$ on the valuation}

For the rest of this subsection, we assume that $\mathcal{O}$ is a valuation ring on $K, \mathrm{~m}$ is its maximal ideal, and val is the corresponding valuation, and we fix some $a_{0}, \ldots, a_{m} \in \mathcal{K}$ such that $\operatorname{val}\left(a_{i}\right) \neq \operatorname{val}\left(a_{j}\right)$ for all $0 \leq i \neq j \leq m$.

This implies in particular that $\left\{\frac{1}{a_{0}}, \ldots, \frac{1}{a_{m}}\right\}$ are $\mathbb{F}_{p^{-}}$-linearly independent (as the valuation of any $\mathbb{F}_{p^{-}}$ linear combination is $\neq \infty$ ). Throughout this section, we let $f:=f_{\bar{a}}=\sum_{j=0}^{m} \alpha_{j} x_{j}$ be the isomorphism $G_{\bar{a}}(\mathbb{K}) \rightarrow(\mathbb{K},+)$ given by Fact 3.13. Let $\alpha_{0}, \ldots, \alpha_{m} \in \mathcal{K}$ be as defined in Subsection 3.2. We will prove several technical lemmas that allow us to control val $\left(f\left(x_{1}, \ldots, x_{m}\right)\right)$ in terms of the tuple $\left(\operatorname{val}\left(x_{1}\right), \ldots, \operatorname{val}\left(x_{m}\right)\right)$, and vice versa. To motivate this analysis, the reader might prefer to check how it is used in the proof of Proposition 3.5 in the next section before going into the details of the calculations here.

Remark 3.14. Assume $\left(x_{0}, \ldots, x_{m}\right) \in G_{\bar{a}}$; then

$$
\operatorname{val}\left(a_{i}\right)+\operatorname{val}\left(x_{i}^{p}-x_{i}\right)=\operatorname{val}\left(a_{j}\right)+\operatorname{val}\left(x_{j}^{p}-x_{j}\right)
$$

for all $0 \leq i, j \leq m$.

Additionally note that

$$
\operatorname{val}\left(x_{i}^{p}-x_{i}\right)=\left\{\begin{aligned}
\operatorname{val}\left(x_{i}\right) & \text { if } \operatorname{val}\left(x_{i}\right)>0 \\
p \operatorname{val}\left(x_{i}\right) & \text { if } \operatorname{val}\left(x_{i}\right)<0
\end{aligned}\right.
$$

and

$$
\operatorname{val}\left(x_{i}^{p}-x_{i}\right) \geq 0 \text { if } \operatorname{val}\left(x_{i}\right)=0 .
$$

Lemma 3.15. Suppose that $0<\operatorname{val}\left(a_{0}\right)<\cdots<\operatorname{val}\left(a_{m}\right)$. Then the sequence $\left(\operatorname{val}\left(\alpha_{i}\right): i \in\{0, \ldots, m\}\right)$ is strictly increasing. 
In fact, for any $0 \leq i \leq m$, we have

$$
\operatorname{val}\left(\alpha_{i}\right)=\frac{1}{p^{m-i}} \operatorname{val}\left(a_{i}\right)+\sum_{j=i}^{m-1} \frac{p-1}{p^{m-j}} \operatorname{val}\left(a_{j+1}\right)>0 .
$$

Proof. Recall that, by linear algebra, for each $0 \leq i \leq m$, we have

$$
\alpha_{i}=\left(A^{-1}\right)_{i, m}=\frac{1}{\operatorname{det}(A)} C_{m, i},
$$

where $C_{m, i}$ is the corresponding cofactor of $A$. That is,

$$
\alpha_{i}=\frac{(-1)^{m+i} \Delta\left(a_{0}^{-\frac{1}{p^{m}}}, \ldots, a_{i-1}^{-\frac{1}{p^{m}}}, a_{i+1}^{-\frac{1}{p^{m}}}, \ldots, a_{m}^{-\frac{1}{p^{m}}}\right)}{\Delta\left(a_{0}^{-\frac{1}{p^{m}}}, \ldots, a_{m}^{-\frac{1}{p^{m}}}\right)} .
$$

Now, we compute the valuation of the numerator and denominator separately. First,

$$
\Delta\left(a_{0}^{-\frac{1}{p^{m}}}, \ldots, a_{m}^{-\frac{1}{p^{m}}}\right)=\sum_{\pi \in \operatorname{Sym}(\{0, \ldots, m\})} \operatorname{sign}(\pi)\left(a_{\pi(0)}^{-\frac{1}{p^{m}}}\right)^{p^{0}} \cdot \ldots \cdot\left(a_{\pi(m)}^{-\frac{1}{p^{m}}}\right)^{p^{m}}
$$

Let $i<j$; then

$$
\operatorname{val}\left(a_{i}{ }^{-\frac{1}{p^{m}}}\right)=-\frac{1}{p^{m}} \operatorname{val}\left(a_{i}\right)>-\frac{1}{p^{m}} \operatorname{val}\left(a_{j}\right)=\operatorname{val}\left(a_{j}{ }^{-\frac{1}{p^{m}}}\right)
$$

Thus $\left(\operatorname{val}\left(a_{i}{ }^{-\frac{1}{p^{m}}}\right): 0 \leq i \leq m\right)$ is strictly decreasing. Using this, we see that

$$
\operatorname{val}\left(\left(a_{0}^{-\frac{1}{p^{m}}}\right)^{p^{0}} \cdot \ldots \cdot\left(a_{m}^{-\frac{1}{p^{m}}}\right)^{p^{m}}\right)<\operatorname{val}\left(\left(a_{\pi(0)}^{-\frac{1}{p^{m}}}\right)^{p^{0}} \cdot \ldots \cdot\left(a_{\pi(m)}^{-\frac{1}{p^{m}}}\right)^{p^{m}}\right)
$$

for every non-identity permutation $\pi \in \operatorname{Sym}(\{0, \ldots, m\})$. Thus

$$
\begin{aligned}
\operatorname{val}\left(\Delta\left(a_{0}^{-\frac{1}{p^{m}}}, \ldots, a_{m}^{-\frac{1}{p^{m}}}\right)\right) & =\operatorname{val}\left(\left(a_{0}^{-\frac{1}{p^{m}}}\right)^{p^{0}} \cdot \ldots \cdot\left(a_{m}^{-\frac{1}{p^{m}}}\right)^{p^{m}}\right) \\
& =-\sum_{j=0}^{m} \frac{1}{p^{m-j}} \operatorname{val}\left(a_{j}\right) .
\end{aligned}
$$

Now we turn to the numerator:

$$
\begin{aligned}
& \Delta\left(a_{0}^{-\frac{1}{p^{m}}}, \ldots, a_{i-1}^{-\frac{1}{p^{m}}}, a_{i+1}^{-\frac{1}{p^{m}}}, \ldots, a_{m}^{-\frac{1}{p^{m}}}\right)= \\
& \sum_{\pi \in \operatorname{Sym}(\{0, \ldots, i-1, i+1, \ldots, m\})} \operatorname{sign}(\pi) \prod_{0 \leq j \leq i-1}\left(a_{\pi(j)}^{-\frac{1}{p^{m}}}\right)^{p^{j}} \cdot \prod_{i+1 \leq j \leq m}\left(a_{\pi(j)}^{-\frac{1}{p^{m}}}\right)^{p^{j-1}} .
\end{aligned}
$$


Again,

$$
\begin{array}{r}
\operatorname{val}\left(\prod_{0 \leq j \leq i-1}\left(a_{j}^{-\frac{1}{p^{m}}}\right)^{p^{j}} \cdot \prod_{i+1 \leq j \leq m}\left(a_{j}^{-\frac{1}{p^{m}}}\right)^{p^{j-1}}\right) \\
<\operatorname{val}\left(\prod_{0 \leq j \leq i-1}\left(a_{\pi(j)}^{-\frac{1}{p^{m}}}\right)^{p^{j}} \cdot \prod_{i+1 \leq j \leq m}\left(a_{\pi(j)}^{-\frac{1}{p^{m}}}\right)^{p^{j-1}}\right)
\end{array}
$$

for every non-identity permutation $\pi \in \operatorname{Sym}(\{0, \ldots, m\})$. Thus

$$
\begin{aligned}
& \operatorname{val}\left(\Delta\left(a_{0}^{-\frac{1}{p^{m}}}, \ldots, a_{i-1}^{-\frac{1}{p^{m}}}, a_{i+1}^{-\frac{1}{p^{m}}}, \ldots, a_{m}^{-\frac{1}{p^{m}}}\right)\right)= \\
& \operatorname{val}\left(\prod_{0 \leq j \leq i-1}\left(a_{j}^{-\frac{1}{p^{m}}}\right)^{p^{j}} \cdot \prod_{i+1 \leq j \leq m}\left(a_{j}^{-\frac{1}{p^{m}}}\right)^{p^{j-1}}\right)= \\
& -\sum_{j=0}^{i-1} \frac{1}{p^{m-j}} \operatorname{val}\left(a_{j}\right)-\sum_{j=i+1}^{m} \frac{1}{p^{m-j+1}} \operatorname{val}\left(a_{j}\right) .
\end{aligned}
$$

Combining, we get

$$
\begin{aligned}
\operatorname{val}\left(\alpha_{i}\right) & =\operatorname{val}\left(\Delta\left(a_{0}^{-\frac{1}{p^{m}}}, \ldots, a_{i-1}^{-\frac{1}{p^{m}}}, a_{i+1}^{-\frac{1}{p^{m}}}, \ldots, a_{m}^{-\frac{1}{p^{m}}}\right)\right)-\operatorname{val}\left(\Delta\left(a_{0}^{-\frac{1}{p^{m}}}, \ldots, a_{m}^{-\frac{1}{p^{m}}}\right)\right) \\
& =-\sum_{j=0}^{i-1} \frac{1}{p^{m-j}} \operatorname{val}\left(a_{j}\right)-\sum_{j=i+1}^{m} \frac{1}{p^{m-j+1}} \operatorname{val}\left(a_{j}\right)-\left(-\sum_{j=0}^{m} \frac{1}{p^{m-j}} \operatorname{val}\left(a_{j}\right)\right) \\
& =-\sum_{j=i+1}^{m} \frac{1}{p^{m-j+1}} \operatorname{val}\left(a_{j}\right)+\frac{1}{p^{m-i}} \operatorname{val}\left(a_{i}\right)+\sum_{j=i+1}^{m} \frac{1}{p^{m-j}} \operatorname{val}\left(a_{j}\right) \\
& =\frac{1}{p^{m-i}} \operatorname{val}\left(a_{i}\right)+\sum_{j=i+1}^{m}\left(\frac{1}{p^{m-j}}-\frac{1}{p^{m-j+1}}\right) \operatorname{val}\left(a_{j}\right) \\
& =\frac{1}{p^{m-i}} \operatorname{val}\left(a_{i}\right)+\sum_{j=i+1}^{m} \frac{p-1}{p^{m-j+1}} \operatorname{val}\left(a_{j}\right) \\
& =\frac{1}{p^{m-i}} \operatorname{val}\left(a_{i}\right)+\sum_{j=i}^{m-1} \frac{p-1}{p^{m-j}} \operatorname{val}\left(a_{j+1}\right) .
\end{aligned}
$$

Remark 3.16. Let $\sigma \in \operatorname{Sym}(\{0,1, \ldots, m\})$ be an arbitrary permutation, and let $\bar{a}^{\prime}:=$ $\left(a_{\sigma(0)}, \ldots, a_{\sigma(m)}\right)$. Let $\bar{\alpha}^{\prime}:=\left(\alpha_{0}^{\prime}, \ldots, \alpha_{m}^{\prime}\right)$ be the tuple given in Fact 3.13 with respect to the tuple $\bar{a}^{\prime}$, so that the map $f_{\bar{a}^{\prime}}: G_{\bar{a}^{\prime}}(\mathbb{K}) \rightarrow(\mathbb{K},+)$ given by $f_{\bar{a}^{\prime}}\left(x_{0}, \ldots, x_{m}\right):=\sum_{j=0}^{m} \alpha_{j}^{\prime} x_{j}$ is a group isomorphism. Then val $\left(\alpha_{i}^{\prime}\right)=\operatorname{val}\left(\alpha_{\sigma(i)}\right)$ for all $0 \leq i \leq m$. 
Proof. As in the proof of Lemma 3.15, for any $0 \leq i \leq m$, we have

$$
\begin{aligned}
\operatorname{val}\left(\alpha_{i}^{\prime}\right) & =\operatorname{val}\left(\frac{\Delta\left(a_{\sigma(0)}^{-\frac{1}{p^{m}}}, \ldots, a_{\sigma(i-1)}^{-\frac{1}{p^{m}}}, a_{\sigma(i+1)}^{-\frac{1}{p^{m}}}, \ldots, a_{\sigma(m)}^{-\frac{1}{p^{m}}}\right)}{\Delta\left(a_{\sigma(0)}^{-\frac{1}{p^{m}}}, \ldots, a_{\sigma(m)}^{-\frac{1}{p^{m}}}\right)}\right) \\
& =\operatorname{val}\left(\frac{(-1)^{\operatorname{sign}\left(\sigma^{\prime}\right)} \Delta\left(a_{0}^{-\frac{1}{p^{m}}}, a_{1}^{-\frac{1}{p^{m}}}, \ldots, a_{\sigma(i)-1}^{-\frac{1}{p^{m}}}, a_{\sigma(i)+1}^{-\frac{1}{p^{m}}}, \ldots, a_{m}^{-\frac{1}{p^{m}}}\right)}{(-1)^{\operatorname{sign}(\sigma)} \Delta\left(a_{0}^{-\frac{1}{p^{m}}}, \ldots, a_{m}^{-\frac{1}{p^{m}}}\right)}\right) \\
& =\operatorname{val}\left(\alpha_{\sigma(i)}\right),
\end{aligned}
$$

where $\sigma^{\prime}:=\sigma \uparrow\{0, \ldots, \sigma(i)-1, \sigma(i)+1, \ldots, m\}$.

Corollary 3.17. Let $0 \leq l \leq m$ be arbitrary. Suppose that $\operatorname{val}\left(a_{s}\right)<\operatorname{val}\left(a_{l}\right)$ for all $0 \leq s \neq l \leq m$; then $\operatorname{val}\left(\alpha_{l}\right)=\operatorname{val}\left(a_{l}\right)$ and $\operatorname{val}\left(\alpha_{s}\right)<\operatorname{val}\left(a_{l}\right)$ for all $0 \leq s \leq m, s \neq l$.

Proof. Since all of the $a_{i}$ 's have different valuations by assumption, reordering and using Remark 3.16 we may assume that $0<\operatorname{val}\left(a_{0}\right)<\cdots<\operatorname{val}\left(a_{m}\right)$ and $l=m$. Then using Lemma 3.15, we immediately obtain the result.

Lemma 3.18. Suppose that $0<\operatorname{val}\left(a_{0}\right)<\cdots<\operatorname{val}\left(a_{m}\right)$, and let $y \in K$ be such that $\operatorname{val}\left(a_{m}\right)<\operatorname{val}(y)$. Let $\left(x_{0}, \ldots, x_{m}\right) \in G_{\bar{a}}(K)$ be such that $f^{-1}(y)=\left(x_{0}, \ldots, x_{m}\right)$. Then $\operatorname{val}\left(x_{m}\right)=\operatorname{val}(y)-\operatorname{val}\left(a_{m}\right)$. In particular, $\operatorname{val}\left(x_{m}\right)>0$.

Proof. By the formula for $f^{-1}$ in Subsection 3.2, we have that

$$
x_{m}=\sum_{j=0}^{m} \beta_{m, j} y^{p^{j}}
$$

where $\beta_{m, j}$ is the $(m, j)$-entry of the inverse of the matrix

$$
D:=\left(\alpha_{j}^{p^{i}}\right)_{i, j}, 0 \leq i, j \leq m
$$

So $\beta_{m, j}=\frac{1}{\operatorname{det} D} C_{j, m}$, where $C_{j, m}$ is the $(j, m)$-cofactor of $D$. We determine the valuation of each of the summands in $(*)$ separately. Fix $0 \leq j \leq m$; then

$$
\operatorname{val}\left(\beta_{m, j} y^{p^{j}}\right)=\operatorname{val}\left(\beta_{m, j}\right)+p^{j} \operatorname{val}(y)
$$

and

$$
\operatorname{val}\left(\beta_{m, j}\right)=\operatorname{val}\left(C_{j, m}\right)-\operatorname{val}(\operatorname{det} D)
$$

Again let us compute the valuations on the right hand side of the above equation separately. First,

$$
\operatorname{val}(\operatorname{det} D)=\operatorname{val}\left(\sum_{\pi \in \operatorname{Sym}(\{0, \ldots, m\})} \operatorname{sign}(\pi) \prod_{i=0}^{m} \alpha_{\pi(i)}^{p^{i}}\right)
$$


As $\operatorname{val}\left(\alpha_{i}\right)$ is strictly increasing with $i$ by Lemma 3.15, note that val $\left(\prod_{i=0}^{m} \alpha_{\pi(i)}^{p^{i}}\right)$ is strictly minimal if $\pi$ maps $i$ to $m-i$ for all $0 \leq i \leq m$. So

$$
\operatorname{val}(\operatorname{det} D)=\operatorname{val}\left(\prod_{i=0}^{m} \alpha_{m-i}^{p^{i}}\right)=\sum_{i=0}^{m} p^{i} \operatorname{val}\left(\alpha_{m-i}\right) .
$$

Similarly,

$$
\operatorname{val}\left(C_{j, m}\right)=\sum_{\pi \in \operatorname{Sym}(\{0, \ldots, m-1\})} \operatorname{sign}(\pi) \prod_{i=0}^{j-1} \alpha_{\pi(i)}^{p^{i}} \cdot \prod_{i=j+1}^{m} \alpha_{\pi(i-1)}^{p^{i}}
$$

Again, as $\operatorname{val}\left(\alpha_{i}\right)$ is strictly increasing with $i$, we conclude that

$$
\operatorname{val}\left(\prod_{i=0}^{j-1} \alpha_{\pi(i)}^{p^{i}} \cdot \prod_{i=j+1}^{m} \alpha_{\pi(i-1)}^{p^{i}}\right)
$$

is strictly minimal if $\pi(i)=(m-1)-i$ for all $0 \leq i \leq m-1$. Hence

$$
\begin{aligned}
\operatorname{val}\left(C_{j, m}\right) & =\operatorname{val}\left(\prod_{i=0}^{j-1} \alpha_{m-1-i}^{p^{i}} \cdot \prod_{i=j+1}^{m} \alpha_{m-i}^{p^{i}}\right) \\
& =\sum_{i=0}^{j-1} p^{i} \operatorname{val}\left(\alpha_{m-1-i}\right)+\sum_{i=j+1}^{m} p^{i} \operatorname{val}\left(\alpha_{m-i}\right)
\end{aligned}
$$

Thus

$$
\begin{aligned}
\operatorname{val}\left(\beta_{m, j}\right) & =\operatorname{val}\left(C_{j, m}\right)-\operatorname{val}(\operatorname{det} D) \\
& =\sum_{i=0}^{j-1} p^{i} \operatorname{val}\left(\alpha_{m-1-i}\right)+\sum_{i=j+1}^{m} p^{i} \operatorname{val}\left(\alpha_{m-i}\right)-\sum_{i=0}^{m} p^{i} \operatorname{val}\left(\alpha_{m-i}\right) \\
& =\sum_{i=0}^{j-1} p^{i} \operatorname{val}\left(\alpha_{m-1-i}\right)-\left(\operatorname{val}\left(\alpha_{m}\right)+\sum_{i=1}^{j} p^{i} \operatorname{val}\left(\alpha_{m-1}\right)\right) \\
& =\sum_{i=0}^{j-1} p^{i} \operatorname{val}\left(\alpha_{m-1-i}\right)-\operatorname{val}\left(\alpha_{m}\right)-\sum_{i=0}^{j-1} p^{i+1} \operatorname{val}\left(\alpha_{m-1-i}\right) \\
& =-\operatorname{val}\left(\alpha_{m}\right)-\sum_{i=0}^{j-1}\left(p^{i+1}-p^{i}\right) \operatorname{val}\left(\alpha_{m-1-i}\right) \\
& =-\operatorname{val}\left(\alpha_{m}\right)-\sum_{i=1}^{j} p^{i-1}(p-1) \operatorname{val}\left(\alpha_{m-i}\right)
\end{aligned}
$$


Note that for any $1 \leq j \leq m$, we have

$$
\begin{aligned}
\operatorname{val}\left(\beta_{m, j-1} y^{p^{j-1}}\right) & =\operatorname{val}\left(\beta_{m, j-1}\right)+p^{j-1} \operatorname{val}(y) \\
& =\operatorname{val}\left(\beta_{m, j}\right)+p^{j-1}(p-1) \operatorname{val}\left(\alpha_{m-j}\right)+p^{j-1} \operatorname{val}(y) \\
& <\operatorname{val}\left(\beta_{m, j}\right)+p^{j} \operatorname{val}(y) \\
& =\operatorname{val}\left(\beta_{m, j} y^{p^{j}}\right) .
\end{aligned}
$$

Thus $\operatorname{val}\left(\beta_{m, 0} y\right)$ is strictly minimal amongst them, so

$$
\operatorname{val}\left(x_{m}\right)=\operatorname{val}\left(\beta_{m, 0} y\right)=-\operatorname{val}\left(\alpha_{m}\right)+\operatorname{val}(y)=-\operatorname{val}\left(a_{m}\right)+\operatorname{val}(y)
$$

using Lemma 3.15.

Lemma 3.19. Let $\left(x_{0}, \ldots, x_{m}\right) \in G_{\bar{a}}(K)$ be arbitrary.

1. Assume that for a fixed $0 \leq l \leq m$, we have that $\operatorname{val}\left(a_{l}\right)>\operatorname{val}\left(a_{s}\right)$ for all $0 \leq s \neq l \leq m$ and $\operatorname{val}\left(x_{l}\right) \geq 0$. Then for any $0 \leq s \neq l \leq m$, if $\operatorname{val}\left(x_{s}\right)>0$, we obtain $\operatorname{val}\left(\alpha_{s} x_{s}\right)>\operatorname{val}\left(\alpha_{l} x_{l}\right)$.

2. Suppose that $0 \leq s \neq t \leq m$ are such that $\operatorname{val}\left(a_{s}\right)<\operatorname{val}\left(a_{t}\right), \operatorname{val}\left(x_{s}\right)=0$ and $\operatorname{val}\left(x_{t}\right) \geq 0$. Then $\operatorname{val}\left(\alpha_{s} x_{s}\right)<\operatorname{val}\left(\alpha_{t} x_{t}\right)$.

Proof. Reordering the $a_{i}$ 's if necessary (relying on Remark 3.16), we may assume that $0<\operatorname{val}\left(a_{0}\right)<$ $\cdots<\operatorname{val}\left(a_{m}\right)$, so in particular $l=m, s<t$ and $\operatorname{val}\left(x_{m}\right) \geq 0$ by assumption.

1. By Remark 3.14 and assumption val $\left(x_{s}\right)>0$, we have

$$
\operatorname{val}\left(x_{s}\right)=\operatorname{val}\left(a_{m}\right)-\operatorname{val}\left(a_{s}\right)+\operatorname{val}\left(x_{m}^{p}-x_{m}\right) .
$$

Moreover, in general we have

$$
\frac{1}{p^{m-s}}+\sum_{s+1 \leq j \leq m} \frac{p-1}{p^{m+1-j}}=1
$$

Then

$$
\begin{aligned}
& \operatorname{val}\left(\alpha_{s} x_{s}\right)=\operatorname{val}\left(\alpha_{s}\right)+\operatorname{val}\left(x_{s}\right) \\
& \stackrel{3.15}{\underset{(\dagger)}{=}} \frac{1}{p^{m-s}} \operatorname{val}\left(a_{s}\right)+\sum_{j=s+1}^{m} \frac{p-1}{p^{m+1-j}} \operatorname{val}\left(a_{j}\right)+\operatorname{val}\left(a_{m}\right)-\operatorname{val}\left(a_{s}\right)+\operatorname{val}\left(x_{m}^{p}-x_{m}\right)
\end{aligned}
$$

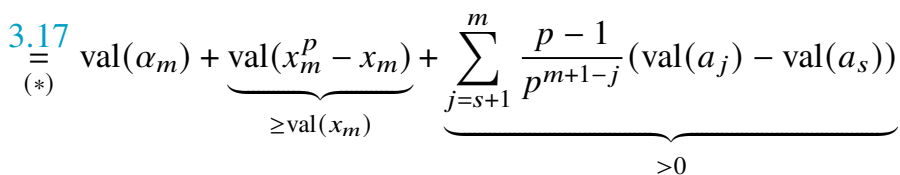

$$
\begin{aligned}
& >\operatorname{val}\left(\alpha_{m}\right)+\operatorname{val}\left(x_{m}\right) \\
& =\operatorname{val}\left(\alpha_{m} x_{m}\right) \text {. }
\end{aligned}
$$

2. By Lemma 3.15 the sequence $\operatorname{val}\left(\alpha_{i}\right)$ 's is strictly increasing. Thus, if $\operatorname{val}\left(x_{s}\right)=0$, then

$$
\begin{aligned}
\operatorname{val}\left(\alpha_{s} x_{s}\right) & =\operatorname{val}\left(\alpha_{s}\right) \\
& 3.15+{ }_{+ \text {ass. }} \operatorname{val}\left(\alpha_{t}\right)+\operatorname{val}\left(x_{t}\right) \\
& =\operatorname{val}\left(\alpha_{t} x_{t}\right) .
\end{aligned}
$$


Lemma 3.20. Let $y \in K$ be such that $\operatorname{val}\left(a_{j}\right)<\operatorname{val}(y)$ for all $0 \leq j \leq m$. Now let $\left(x_{0}, \ldots, x_{m}\right) \in G_{\bar{a}}(K)$ be equal to $f^{-1}(y)$. Then $\operatorname{val}\left(x_{j}\right)>0$ for all $0 \leq j \leq m$.

Proof. Up to reordering the $a_{j}$ 's (using Remark 3.16), we may assume that $0<\operatorname{val}\left(a_{0}\right)<\cdots<\operatorname{val}\left(a_{m}\right)$. Then, $\operatorname{val}\left(x_{m}\right)>0$ by Lemma 3.18, and $\left(\operatorname{val}\left(\alpha_{j}\right): 0 \leq j \leq m\right)$ is strictly increasing by Lemma 3.15. For any $0 \leq j \leq m$, by Remark 3.14, we have

$$
\operatorname{val}\left(x_{j}^{p}-x_{j}\right)=\operatorname{val}\left(a_{m}\right)-\operatorname{val}\left(a_{j}\right)+\operatorname{val}\left(x_{m}^{p}-x_{m}\right)>0,
$$

and thus $\operatorname{val}\left(x_{j}\right) \geq 0$.

If $\operatorname{val}\left(x_{j}\right)>0$ for all $j$, we are done. Otherwise, let $I=\left\{1 \leq j \leq m: \operatorname{val}\left(x_{j}\right)=0\right\} \neq \emptyset$, and let $j_{*}:=\min \{I\}$.

Claim 1. $\operatorname{val}\left(\alpha_{j_{*}} x_{j_{*}}\right)$ is strictly minimal in $\left\{\operatorname{val}\left(\alpha_{j} x_{j}\right): 1 \leq j \leq m\right\}$.

Proof. Assume first $j \in I \backslash\left\{j_{*}\right\}$; then $\operatorname{val}\left(x_{j}\right)=0$ and $j>j_{*}$. Hence

$$
\operatorname{val}\left(\alpha_{j_{*}} x_{j_{*}}\right)=\operatorname{val}\left(\alpha_{j_{*}}\right)<\operatorname{val}\left(\alpha_{j}\right)=\operatorname{val}\left(\alpha_{j} x_{j}\right)
$$

Otherwise $j \notin I-$ that is, $\operatorname{val}\left(x_{j}\right)>0$. Then

$$
\operatorname{val}\left(\alpha_{j} x_{j}\right) \stackrel{3.19(1)}{>} \operatorname{val}\left(\alpha_{m} x_{m}\right) \stackrel{3.19(2)}{>} \operatorname{val}\left(\alpha_{j_{*}} x_{j_{*}}\right)
$$

Thus $\operatorname{val}(y)=\operatorname{val}\left(\sum_{j=0}^{m} \alpha_{j} x_{j}\right)=\operatorname{val}\left(\alpha_{j_{*}} x_{j_{*}}\right)=\operatorname{val}\left(\alpha_{j_{*}}\right) \stackrel{3.17}{<} \operatorname{val}\left(a_{m}\right)<\operatorname{val}(y)$, which yields a contradiction. Hence $\operatorname{val}\left(x_{j}\right)>0$ for all $0 \leq j \leq m$.

\subsection{Proof of Proposition 3.5}

We may assume that $\left(K, \mathcal{O}_{1}, \mathcal{O}_{2}\right)$ is $\boldsymbol{\aleph}_{0}$-saturated. Let $\mathbb{K}$ be the algebraic closure of $K$, and let $\mathcal{K}:=$ $\bigcap_{n \in \mathbb{N}} K^{\frac{1}{p^{n}}}$ be the largest perfect subfield of $K$. Let $\ell$ be the natural number given by Proposition 3.9 for the uniformly defined subgroups $x_{0} \cdot \ldots \cdot x_{n-1} \cdot \wp(K)$ and $x_{0} \cdot \ldots \cdot x_{n-1} \cdot \wp(J)$ of $(K,+)$.

Let $y \in J$ be arbitrary. We will show that it has an Artin-Schreier root in $J$. We stress that this element $y$ will be fixed until the end of the proof, and several additional parameters depending on this $y$ will be chosen in the course of the proof.

Claim 3.21. There exists an infinite sequence $\left(d_{i}\right)_{i \in \mathbb{N}}$ of elements of $\mathcal{K}$ such that $0<k \cdot \operatorname{val}_{t}\left(d_{i+1}\right)<$ $\operatorname{val}_{t}\left(d_{i}\right)<\operatorname{val}_{t}(y)$ holds for all $i, k \in \mathbb{N}$ and both $t \in\{1,2\}$ simultaneously.

Proof. Let $e_{0}:=y$, and we define the elements $e_{k} \in J$ by induction on $k \in \mathbb{N}_{\geq 1}$ as follows. Assume $e_{k}$ for $k \in \mathbb{N}$ is given. As the field $K$ is Artin-Schreier closed by Fact 3.4, we let $e \in K$ be an Artin-Schreier root of $\frac{1}{e_{k}}-$ that is, $\frac{1}{e_{k}}=e^{p}-e$. For any $t \in\{1,2\}$, we have $\operatorname{val}_{t}\left(e_{k}\right)>0$ by assumption, hence $\operatorname{val}_{t}\left(\frac{1}{e_{k}}\right)<0$. Consequently $\operatorname{val}_{t}\left(e^{p}-e\right)<0$ as well as $\operatorname{val}_{t}(e)<0$, and so $\operatorname{val}_{t}\left(e^{p}-e\right)=p \operatorname{val}_{t}(e)$. Hence, letting $e_{k+1}:=\frac{1}{e}$, we obtain that

$$
\operatorname{val}_{t}\left(e_{k}\right)=-\operatorname{val}_{t}\left(\frac{1}{e_{k}}\right)=-p \operatorname{val}_{t}(e)=p \operatorname{val}_{t}\left(\frac{1}{e}\right)=p \operatorname{val}_{t}\left(e_{k+1}\right) .
$$

It follows from the construction that for every $k \in \mathbb{N}_{\geq 1}$ the elements $e_{k} \in K$ satisfy $0<\operatorname{val}_{t}\left(e_{k}\right)=$ $\frac{1}{p^{k}} \operatorname{val}_{t}(y)<\operatorname{val}_{t}(y)$ for both $t \in\{1,2\}$ simultaneously. Let now $m, k \in \mathbb{N}$ be arbitrary, and we define 
$d_{i}:=e_{i k+m}^{p^{m}}$ for $i \in \mathbb{N}$. Note that

$$
\begin{aligned}
\operatorname{val}_{t}\left(d_{i}\right) & =\operatorname{val}_{t}\left(e_{i k+m}^{p^{m}}\right)=p^{m} \operatorname{val}_{t}\left(e_{i k+m}\right)=p^{m} \frac{1}{p^{i k+m}} \operatorname{val}_{t}(y) \\
& =\frac{p^{k}}{p^{k}} p^{m} \frac{1}{p^{i k+m}} \operatorname{val}_{t}(y)=p^{k} p^{m} \frac{1}{p^{(i+1) k+m}} \operatorname{val}_{t}(y) \\
& =p^{k} p^{m} \operatorname{val}_{t}\left(e_{(i+1) k+m}\right)=p^{k} \operatorname{val}_{t}\left(e_{(i+1) k+m}^{p^{m}}\right) \\
& =p^{k} \operatorname{val}_{t}\left(d_{i+1}\right)
\end{aligned}
$$

and $p^{k-1} \operatorname{val}_{t}\left(d_{i}\right)=\frac{p^{k-1}}{p^{i k}} \operatorname{val}_{t}(y)<\operatorname{val}_{t}(y)$ for all $i \in \mathbb{N}$. Then:

$\circ d_{i} \in K^{\frac{1}{p^{m}}}$ for all $i \in \mathbb{N}$.

○ For each $t \in\{1,2\}$ and all $i \in \mathbb{N}$, we have $0<p^{k-1} \cdot \operatorname{val}_{t}\left(d_{i+1}\right)<\operatorname{val}_{t}\left(d_{i}\right)<\operatorname{val}_{t}(y)$.

As $m, k$ were arbitrary and $\mathcal{K}$ is type-definable over $\emptyset$, the claim follows by saturation of $\left(K, \mathcal{O}_{1}, \mathcal{O}_{2}\right)$.

Now let $\left(d_{i}\right)_{i \in \mathbb{N}}$ be a sequence in $\mathcal{K}$ given by Claim 3.21. Then we can choose from it elements $\left\{b_{j, l}: j<n, l<\ell\right\}$ in $\mathcal{K}$ such that for all $j<n, l<\ell$, we have

$\circ \operatorname{val}_{t}\left(b_{n-1, l}\right)<\operatorname{val}_{t}\left(b_{0, l+1}\right)$

$\circ 0<(j+1) \cdot \operatorname{val}_{t}\left(b_{j, l}\right)<\operatorname{val}_{t}\left(b_{j, l+1}\right)$

$\circ n \cdot \operatorname{val}_{t}\left(b_{n-1, \ell-1}\right)<\operatorname{val}_{t}(y)$

for both $t \in\{1,2\}$ simultaneously. For each $\left(l_{0}, \ldots, l_{n-1}\right) \in \ell^{n}$, we define

$$
b_{l_{0}, \ldots, l_{n-1}}:=\prod_{j=0}^{n-1} b_{j, l_{j}} \in \mathcal{K}
$$

Claim 3.22. For each $t \in\{1,2\}$, we have:

$\circ 0<\operatorname{val}_{t}\left(b_{l_{0}, \ldots, l_{n-1}}\right)<\operatorname{val}_{t}(y)$ for all $\left(l_{0}, \ldots, l_{n-1}\right) \in \ell^{n}$

$\circ \operatorname{val}_{t}\left(b_{l_{0}, \ldots, l_{n-1}}\right)<\operatorname{val}_{t}\left(b_{p_{0}, \ldots, p_{n-1}}\right)$ if and only if $\left(l_{n-1}, \ldots, l_{0}\right)<_{\text {lex }}\left(p_{n-1}, \ldots, p_{0}\right)$

Proof. The first item is clear by the choice of $b_{j, l}$, and we check the second one. Assume $\left(l_{n-1}, \ldots, l_{0}\right)<_{\operatorname{lex}}\left(p_{n-1}, \ldots, p_{0}\right)$, and let $0 \leq j^{*}<n$ be maximal such that $l_{j^{*}}<p_{j^{*}}$. Then by the choice of $b_{j, l}$, we have

$$
\begin{aligned}
& \operatorname{val}_{t}\left(b_{l_{0}, \ldots, l_{n-1}}\right)=\sum_{j=0}^{n-1} \operatorname{val}_{t}\left(b_{j, l_{j}}\right)=\sum_{j=0}^{j^{*}} \operatorname{val}_{t}\left(b_{j, l_{j}}\right)+\sum_{j=j^{*}+1}^{n-1} \operatorname{val}_{t}\left(b_{j, p_{j}}\right) \\
& \leq\left(j^{*}+1\right) \cdot \operatorname{val}_{t}\left(b_{j^{*}, l_{j^{*}}}\right)+\sum_{j=j^{*}+1}^{n-1} \operatorname{val}_{t}\left(b_{j, p_{j}}\right)<\operatorname{val}_{t}\left(b_{j^{*}, p_{j^{*}}}\right)+\sum_{j=j^{*}+1}^{n-1} \operatorname{val}_{t}\left(b_{j, p_{j}}\right) \\
& \leq \sum_{j=0}^{n-1} \operatorname{val}_{t}\left(b_{j, p_{j}}\right)=\operatorname{val}_{t}\left(b_{p_{0}, \ldots, p_{n-1}}\right)
\end{aligned}
$$

By the choice of $\ell$ and Proposition 3.9, there must exist some $\left(l_{0}^{*}, \ldots, l_{n-1}^{*}\right) \in \ell^{n}$ such that

$$
\bigcap_{\substack{\left(l_{0}, \ldots, l_{n-1}\right) \in \ell^{n} \\ .35 \text { Published online by Cambridge University Press }}} b_{l_{0}, \ldots, l_{n-1}} \cdot \wp(J)=\bigcap_{\left(l_{0}, \ldots, l_{n-1}\right) \in \ell^{n} \backslash\left\{\left(l_{0}^{*}, \ldots, l_{n-1}^{*}\right)\right\}} b_{l_{0}, \ldots, l_{n-1}} \cdot \wp(J),
$$




$$
\bigcap_{\left(l_{0}, \ldots, l_{n-1}\right) \in \ell^{n}} b_{l_{0}, \ldots, l_{n-1}} \cdot \wp(K)=\bigcap_{\left(l_{0}, \ldots, l_{n-1}\right) \in \ell^{n} \backslash\left\{\left(l_{0}^{*}, \ldots, l_{n-1}^{*}\right)\right\}} b_{l_{0}, \ldots, l_{n-1}} \cdot \wp(K) .
$$

Let now $m:=\ell^{n}-1$. The following is straightforward:

Claim 3.23. There exists a tuple $\left(a_{j}: 0 \leq j \leq m\right)$ enumerating the set

$$
\left\{b_{l_{0}, \ldots, l_{n-1}}:\left(l_{0}, \ldots, l_{n-1}\right) \in \ell^{n}\right\}
$$

so that the following holds:

1. $\bigcap_{j=0}^{m} a_{j} \wp(J)=\bigcap_{j=0}^{m-1} a_{j} \wp(J)$;

2. $\bigcap_{j=0}^{m} a_{j} \wp(K)=\bigcap_{j=0}^{m-1} a_{j} \wp(K)$;

3. $0<\operatorname{val}_{t}\left(a_{0}\right)<\cdots<\operatorname{val}_{t}\left(a_{m-1}\right)$ and $\left(\operatorname{val}_{t}\left(a_{j}\right)\right)_{0 \leq j \leq m}$ are pairwise distinct, for both $t \in\{1,2\}$.

We fix such a tuple $\left(a_{j}: 0 \leq j \leq m\right)$ for the rest of the proof. Note that we still have $a_{j} \in \mathcal{K}$ and $0<\operatorname{val}_{t}\left(a_{j}\right)<\operatorname{val}_{t}(y)$ for any $0 \leq j \leq m$ and $t \in\{1,2\}$ by the choice of the elements $b_{l_{0}, \ldots, l_{n-1}}$ and Claim 3.22. Let $\bar{a}:=\left(a_{0}, \ldots, a_{m}\right)$ and $\bar{a}^{\prime}:=\left(a_{0}, \ldots, a_{m-1}\right)$; both tuples satisfy the assumption of Subsection 3.3. Then the following diagram is commutative

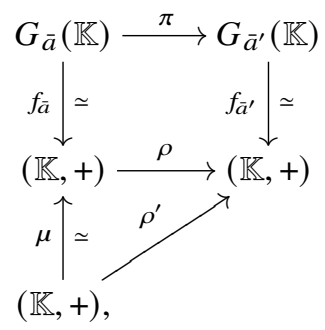

where $\pi$ is the natural projection $\left(x_{0}, \ldots, x_{m}\right) \mapsto\left(x_{0}, \ldots, x_{m-1}\right)$; the maps

$$
\begin{gathered}
f_{\bar{a}}: G_{\bar{a}}(\mathbb{K}) \rightarrow(\mathbb{K},+), \bar{x}=\left(x_{0}, \ldots, x_{m}\right) \mapsto \sum_{j=0}^{m} \alpha_{j} x_{j}, \text { and } \\
f_{\bar{a}^{\prime}}: G_{\bar{a}^{\prime}}(\mathbb{K}) \rightarrow(\mathbb{K},+), \bar{x}=\left(x_{0}, \ldots, x_{m-1}\right) \mapsto \sum_{j=0}^{m-1} \alpha_{j}^{\prime} x_{j}
\end{gathered}
$$

with $\bar{\alpha}=\left(\alpha_{j}: 0 \leq j \leq m\right), \bar{\alpha}^{\prime}=\left(\alpha_{j}^{\prime}: 0 \leq j \leq m-1\right)$ in $\mathcal{K}$ are the isomorphisms given by Fact 3.13 for $\bar{a}$ and $\bar{a}^{\prime}$ respectively; $\rho$ is the algebraic morphism over $\mathcal{K}$ that makes the rectangle commute; and $\mu, \rho^{\prime}:(\mathbb{K},+) \rightarrow(\mathbb{K},+)$ is given by

$$
\mu(t):=\alpha_{m} \cdot t, \quad \rho^{\prime}(t):=\rho\left(\alpha_{m} t\right) .
$$

Note that all these groups and morphisms are defined over $\mathcal{K} \subseteq K$; hence the diagram still commutes with $\mathbb{K}$ replaced by $K$.

We recall the following general fact about additive polynomials.

Fact 3.24 [31, Remark 4.2]. Let $F$ be an algebraically closed fields, and $g: F \rightarrow F$ an additive polynomial $\left(\right.$ i.e. $g\left(t+t^{\prime}\right)=g(t)+g\left(t^{\prime}\right)$ for all $\left.t, t^{\prime} \in F\right)$ with $\operatorname{ker}(g)=\mathbb{F}_{p}$. Then $g=c \cdot\left(t^{p}-t\right)^{p^{k}}$ for some $k \in \mathbb{N}$ and $c \in F$.

Using it, as in the proof of [31, Theorem 4.3], we get the following explicit expression for $\rho^{\prime}$ (we include a proof for completeness). 
Claim 3.25. $\rho^{\prime}(t)=c\left(t^{p}-t\right)$ for some $c \in \mathcal{K}$.

Proof. We have $\pi \uparrow_{G_{\bar{a}}(K)}$ is onto $G_{\bar{a}^{\prime}}(K)$ by Claim 3.22(1); hence $\rho \uparrow_{K}$ is onto as well. From the diagram, we have $|\operatorname{ker}(\rho)|=|\operatorname{ker}(\pi)|=p$, and $\rho$ is an algebraic group morphism of $(\mathbb{K},+)$; hence an additive polynomial. Note that $0 \neq \alpha_{m} \in \operatorname{ker}(\rho)$ as $f_{\bar{a}}^{-1}\left(\alpha_{m}\right)=(0, \ldots, 0,1) \in K^{m+1}$, and $\pi((0, \ldots, 0,1))=(0, \ldots, 0) \in K^{m}$. It follows that $\rho^{\prime}: \mathbb{K} \rightarrow \mathbb{K}$ is also an additive polynomial, with $\operatorname{ker}\left(\rho^{\prime}\right)=\mathbb{F}_{p}$. By Fact 3.24 we have $\rho^{\prime}(t)=c \cdot\left(t^{p}-t\right)^{p^{k}}$ for some some $c \in \mathbb{K}$ and $k \in \mathbb{N}$. In fact, $c \in \mathcal{K}$ as $\alpha_{m} \in \mathcal{K}$ and $\rho$ is over $\mathcal{K}$. Finally, we must have $k=0$ as the degree of $\pi$ as an algebraic morphism is $p$; hence the degree of $\rho^{\prime}$ is also $p$ as the vertical arrows $f_{\bar{a}}, f_{\bar{a}^{\prime}}, \mu$ are algebraic isomorphisms.

We fix $c \in \mathcal{K}$ given by Claim 3.25 for the rest of the proof. The following is a crucial claim relying on the analysis of the effect of the special isomorphisms $f_{\bar{a}}$ on the valuation in Section 3.3.

Claim 3.26. Let $u \in K$ be arbitrary with $\operatorname{val}_{t}(u)>\max \left\{\operatorname{val}_{t}\left(a_{m-1}\right), \operatorname{val}_{t}\left(a_{m}\right)\right\}$ for both $t \in\{1,2\}$. Then there exists some $w \in J$ with $\operatorname{val}_{t}(w)<\operatorname{val}_{t}(u)$ for both $t \in\{1,2\}$ and such that $\rho^{\prime}(w)=u$.

Proof. Let $\left(x_{0}, \ldots, x_{m-1}\right):=\left(f_{\bar{a}^{\prime}}\right)^{-1}(u) \in G_{\bar{a}^{\prime}}(K)$. Note that

$$
0<\operatorname{val}_{t}\left(a_{0}\right)<\ldots<\operatorname{val}_{t}\left(a_{m-1}\right)<\operatorname{val}_{t}(u)
$$

for both $t \in\{1,2\}$ by Claim 3.23(3) and assumption, hence by Lemma 3.20 we have that $\operatorname{val}_{t}\left(x_{j}\right)>0$ for all $t \in\{1,2\}$ and $0 \leq j \leq m-1$. Whence $x_{j} \in J$ for all $0 \leq j \leq m-1$. By Claim 3.23(1) there is some $x_{m} \in J$ such that $a_{m}\left(x_{m}^{p}-x_{m}\right)=a_{j}\left(x_{j}^{p}-x_{j}\right)$ for all $0 \leq j<m$. So there is a preimage of $\left(x_{0}, \ldots, x_{m-1}\right)$ under $\pi$, namely $\left(x_{0}, \ldots, x_{m-1}, x_{m}\right)$, which lies in $J^{m+1}$. Now let

$$
w:=\alpha_{m}^{-1} f\left(x_{0}, \ldots, x_{m-1}, x_{m}\right)=\alpha_{m}^{-1} \sum_{j=0}^{m} \alpha_{j} x_{j},
$$

thus $w$ is a preimage of $u$ under $\rho^{\prime}$. Then for each $t \in\{1,2\}$, we have

$$
\begin{aligned}
& \operatorname{val}_{t}(w)=\operatorname{val}_{t}\left(\alpha_{m}^{-1} \sum_{j=0}^{m} \alpha_{j} x_{j}\right) \\
& =-\operatorname{val}_{t}\left(\alpha_{m}\right)+\operatorname{val}_{t}\left(\sum_{j=0}^{m} \alpha_{j} x_{j}\right) \\
& \text { 3.19(1) }\left\{\begin{array}{cl}
-\operatorname{val}_{t}\left(\alpha_{m}\right)+\operatorname{val}_{t}\left(\alpha_{m}\right)+\operatorname{val}_{t}\left(x_{m}\right) & \text { if } \operatorname{val}_{t}\left(a_{m-1}\right)<\operatorname{val}_{t}\left(a_{m}\right) \\
-\operatorname{val}_{t}\left(\alpha_{m}\right)+\operatorname{val}_{t}\left(\alpha_{m-1}\right)+\operatorname{val}_{t}\left(x_{m-1}\right) & \text { if } \operatorname{val}_{t}\left(a_{m}\right)<\operatorname{val}_{t}\left(a_{m-1}\right)
\end{array}\right. \\
& >0 \text {, }
\end{aligned}
$$

where the last inequality is by Remark 3.16, Lemma 3.15 and Lemma 3.18. Also, by Lemma 3.18 with respect to $\bar{a}^{\prime}$, we have $\operatorname{val}_{t}\left(x_{m-1}\right)=\operatorname{val}_{t}(u)-\operatorname{val}_{t}\left(a_{m-1}\right)$; hence

$$
\operatorname{val}_{t}(u)=\operatorname{val}_{t}\left(a_{m-1}\right)+\operatorname{val}_{t}\left(x_{m-1}\right) \stackrel{3.14}{=} \operatorname{val}_{t}\left(a_{m}\right)+\operatorname{val}_{t}\left(x_{m}\right) .
$$

If $\operatorname{val}_{t}\left(a_{m-1}\right)<\operatorname{val}_{t}\left(a_{m}\right)$, then we have

$$
\begin{aligned}
\operatorname{val}_{t}(u) & =\operatorname{val}_{t}\left(a_{m}\right)+\operatorname{val}_{t}\left(x_{m}\right) \stackrel{3.17}{=} \operatorname{val}_{t}\left(\alpha_{m}\right)+\operatorname{val}_{t}\left(x_{m}\right) \\
& >-\operatorname{val}_{t}\left(\alpha_{m}\right)+\operatorname{val}_{t}\left(\alpha_{m}\right)+\operatorname{val}_{t}\left(x_{m}\right)=\operatorname{val}_{t}(w) .
\end{aligned}
$$


If $\operatorname{val}_{t}\left(a_{m}\right)<\operatorname{val}_{t}\left(a_{m-1}\right)$, then we have

$$
\begin{aligned}
\operatorname{val}_{t}(u) & =\operatorname{val}_{t}\left(a_{m-1}\right)+\operatorname{val}_{t}\left(x_{m-1}\right) \stackrel{3.17}{=} \operatorname{val}_{t}\left(\alpha_{m-1}\right)+\operatorname{val}_{t}\left(x_{m-1}\right) \\
& >-\operatorname{val}_{t}\left(\alpha_{m}\right)+\operatorname{val}_{t}\left(\alpha_{m-1}\right)+\operatorname{val}_{t}\left(x_{m-1}\right)=\operatorname{val}_{t}(w) .
\end{aligned}
$$

In either case, we obtain $\operatorname{val}_{t}(u)>\operatorname{val}_{t}(w)$.

Let now $w$ be as given by Claim 3.26 for $u:=y$. Then for both $t \in\{1,2\}$, we have

$$
\operatorname{val}_{t}(c)=\operatorname{val}_{t}(y)-\operatorname{val}_{t}\left(w^{p}-w\right)=\operatorname{val}_{t}(y)-\operatorname{val}_{t}(w)>0 .
$$

Then $\operatorname{val}_{t}(c y)>\max \left\{\operatorname{val}_{t}\left(a_{m-1}\right), \operatorname{val}_{t}\left(a_{m}\right)\right\}$. Let $w^{\prime} \in J$ be as given by Claim 3.26 applied to $u:=c y$; then

$$
c y=\rho^{\prime}\left(w^{\prime}\right)=c\left(\left(w^{\prime}\right)^{p}-w^{\prime}\right),
$$

that is, $y=\left(w^{\prime}\right)^{p}-w^{\prime}$. Thus $w^{\prime}$ is an Artin-Schreier root of $y$ in $J$. As $y \in J$ was arbitrary, this finishes the proof of Proposition 3.5.

\subsection{Generic multi-ordered/multi-valued fields}

An analog of Corollary 3.6 for valued fields of characteristic 0 currently appears out of reach. In this section, we at least provide some evidence toward it by demonstrating that the model-companion of the theory of fields with several valuations and orderings introduced by van den Dries [45, Chapter III] is not $n$-dependent for any $n$. We use Johnson's PhD thesis [30, Chapter 11] as our reference.

Fix $k \in \mathbb{N}$. For each $1 \leq i \leq k$, let $T_{i}$ be one of the theories ACVF (Algebraically Closed Valued Fields), RCF (Real Closed Fields), or pCF ( $p$-adically Closed Fields), and let $\mathcal{L}_{i}$ denote the language of $T_{i}$ and $\mathcal{L}_{i} \cap \mathcal{L}_{j}=\mathcal{L}_{\text {rings }}$ (i.e. $\mathcal{L}_{i}$ additionally contains a binary predicate $x<_{i} y$ if $T_{i}$ is RCF, or $\operatorname{val}_{i}(x)<\operatorname{val}_{i}(y)$ if $T_{i}$ is ACVF or pCF). Let $\mathcal{L}:=\bigcup_{i=1}^{k} \mathcal{L}_{i}$, and let $T_{0}:=\bigcup_{i=1}^{k}\left(T_{i}\right)_{\forall}$

Fact 3.27 [30, Theorem 11.2.3]. The theory $T_{0}$ has a model companion $T$, and $K \models T_{0}$ is a model of $T$ if:

1. $K$ is existentially closed with respect to finite extensions: that is, if $L$ is a finite algebraic extension of $K$ and $L \models T_{0}$, then $L=K$.

2. For any $m$, let $V$ be an $m$-dimensional absolutely irreducible variety over $K$. For $1 \leq i \leq k$, let $\varphi_{i}(x)$ be a $V$-dense quantifier-free $\mathcal{L}_{i}$-formula with parameters from $K$. Then $\bigcap_{i=1}^{k} \varphi_{i}(K) \neq \emptyset$.

(Where ' $V$-dense' means that $\varphi_{i}(K)$ is Zariski-dense in $V\left(K^{\mathrm{alg}}\right)$; see [30, Section 11.1.1].)

We use the following result established in the proof of [30, Claim 11.5.2].

Fact 3.28. Let $K \models T$. For each $i$, let $\chi_{i}(y)$ be the formula

1. $y>_{i} 0$ if $T_{i}$ is RCF.

2. $\operatorname{val}_{i}\left(y-\frac{1}{4}\right)>0$ if $T_{i}$ is $\mathrm{ACVF}$ or $\mathrm{pCF}$.

Let $\chi(y):=\bigwedge_{i=1}^{k} \chi_{i}(y)$. Then $\chi(K)$ is infinite, and there exists some L-formula $\psi(x, y)$ such that for any $m \in \mathbb{N}$, any $a_{1}, \ldots, a_{m} \in \chi(K)$ pairwise distinct and any $A \subseteq\{1, \ldots, m\}$, there exists some $b$ such that $=\psi\left(b, a_{j}\right) \Longleftrightarrow j \in A$.

This immediately implies that $T$ is not dependent, and we show that the argument can be generalized to show that $T$ is not $n$-dependent for any $n$ as follows.

Proposition 3.29. $T$ is not $n$-dependent for any $n \geq 1$.

Proof. Let $K \models T$ be a saturated model. Fix $n \in \mathbb{N}$. Note that it is enough to find some sequences $\left(c_{\alpha_{1}}^{1}, \ldots, c_{\alpha_{n}}^{n}: \alpha_{1}, \ldots, \alpha_{n} \in \omega\right)$ of elements in $K$ and $e \in K$ such that all elements in the set 
$\left\{c_{\alpha_{1}}^{1} \cdot \ldots \cdot c_{\alpha_{n}}^{n}+e: \alpha_{1}, \ldots, \alpha_{n} \in \omega\right\}$ are pairwise distinct and satisfy $\chi(y)$, as then the formula $\psi^{\prime}\left(x ; y_{1}, \ldots, y_{n}\right)=\psi\left(x ; y_{1} \cdot \ldots \cdot y_{n}+e\right)$ with $\psi$ given by Fact 3.28 is not $n$-dependent.

Since the formulas $\operatorname{val}_{i}\left(\frac{1}{4}-x\right)>0$ and $x>_{i} 0$ are $V$-dense for $V=\mathbb{A}^{1}$, by Fact 3.27(2) we can choose $e \in K$ such that $0<\operatorname{val}_{i}\left(\frac{1}{4}-e\right)<\infty$ for all $1 \leq i \leq k$ for which $T_{i}$ is ACVF or pCF, and $e>_{i} 0$ for all $i$ for which $T_{i}$ is RCF. Let $\gamma_{i}:=\operatorname{val}_{i}\left(\frac{1}{4}-e\right)$.

By induction on $1 \leq t \leq n$, we choose, using saturation of $K$, sequences $\left(c_{\alpha}^{t}: \alpha \in \omega\right)$ in $K$ such that the following holds for each $1 \leq i \leq k$ :

1. If $T_{i}$ is ACVF or pCF:

(a) $\operatorname{val}_{i}\left(c_{\alpha+1}^{t}\right)>n \cdot \operatorname{val}_{i}\left(c_{\alpha}^{t}\right)>\gamma_{i}$ for all $1 \leq t \leq n$ and $\alpha \in \omega$.

(b) $\operatorname{val}_{i}\left(c_{\alpha}^{t+1}\right)>\operatorname{val}_{i}\left(c_{\beta}^{t}\right)$ for all $1 \leq t \leq n-1$ and $\alpha, \beta \in \omega$.

2. If $T_{i}$ is RCF:

(a) $c_{\alpha+1}^{t}>_{i}\left(c_{\alpha}^{t}\right)^{n}>0$ and for all $1 \leq t \leq n$ and $\alpha, \beta \in \omega$.

(b) $c_{\alpha}^{t+1}>_{i} c_{\beta}^{t}$ for all $1 \leq t \leq n-1$ and $\alpha, \beta \in \omega$.

To choose an element $c_{\alpha}^{t} \in K$, we only need to satisfy finitely many quantifier-free formulas with parameters from $\left\{c_{\beta}^{s}: s<t \vee(s=t \wedge \beta<\alpha)\right\} \subseteq K$. All of these are implied by a single condition of the form $\operatorname{val}_{i}(x)>\operatorname{val}_{i}(c)$ or $x>_{i} c$ for each $i$ and some $c \in K$. Thus they can be satisfied in $K$ by Fact $3.27(2)$ since these formulas are $V$-dense for $V=\mathbb{A}^{1}$.

Assume first that $T_{i}$ is $\mathrm{ACVF}$ or $\mathrm{pCF}$ for some $1 \leq i \leq k$. Note that in this case, for any $\left(\alpha_{1}, \ldots, \alpha_{n}\right) \in \omega^{n}$, we have $\operatorname{val}_{i}\left(\prod_{t=1}^{n} c_{\alpha_{t}}^{t}\right)=\sum_{t=1}^{n} \operatorname{val}_{i}\left(c_{\alpha_{t}}^{t}\right)>\gamma_{i}=\operatorname{val}_{i}\left(\frac{1}{4}-e\right)$ by $1(\mathrm{a})$. Then $\operatorname{val}_{i}\left(\left(\prod_{t=1}^{n} c_{\alpha_{t}}^{t}+e\right)-\frac{1}{4}\right)=\operatorname{val}_{i}\left(\prod_{t=1}^{n} c_{\alpha_{t}}^{t}-\left(\frac{1}{4}-e\right)\right)=\operatorname{val}_{i}\left(\frac{1}{4}-e\right)=\gamma_{i}>0$, and therefore $\vDash \chi_{i}\left(\prod_{t=1}^{n} c_{\alpha_{t}}^{t}+e\right)$.

As in the proof of Claim 3.22, we get that $\left(\alpha_{1}, \ldots, \alpha_{n}\right)<\left(\beta_{1}, \ldots, \beta_{n}\right)$ in the lexicographic ordering on $\omega^{n}$ if and only if

$$
\operatorname{val}_{i}\left(\prod_{t=1}^{n} c_{\alpha_{t}}^{t}\right)<\operatorname{val}_{i}\left(\prod_{t=1}^{n} c_{\alpha_{t}}^{t}\right),
$$

so in particular $\prod_{t=1}^{n} c_{\alpha_{t}}^{t}+e \neq \prod_{t=1}^{n} c_{\beta_{t}}^{t}+e$; hence all these elements are pairwise distinct.

Otherwise $T_{i}$ must be RCF for all $1 \leq i \leq k$. Then a similar calculation using 2(a) and 2(b) shows that $\prod_{t=1}^{n} c_{\alpha_{t}}^{t}+e \vDash \chi_{i}$ and that all these elements are pairwise distinct.

\section{Connected components of $n$-dependent groups}

\subsection{Connected components of (type-)definable groups}

We begin by recalling some facts about model-theoretic connected components of (type-)definable groups and state the main theorem of the section.

Definition 4.1. Let $T$ be an arbitrary theory, $G=G(\mathbb{M})$ an $\emptyset$-definable group and $A \subseteq \mathbb{M}$ a small set of parameters. Then $G_{A}^{0}$ is the intersection of all $A$-definable subgroups of $G$ of finite index, and $G_{A}^{00}$ is the intersection of all subgroups of $G$ that are type-definable over $A$ and of bounded index.

As $A$ is small, $G_{A}^{0}$ and $G_{A}^{00}$ are type-definable subgroups of $G$ of bounded index. In fact, we have the following standard results (see e.g. [20, Remark 3.5(2) and Lemma 2.2]).

Fact 4.2. If $H$ is a type-definable subgroup of $G=G(\mathbb{M})$ of bounded index, then it can be written as an intersection of subgroups of $G$ of bounded index, each of which is defined by a partial type consisting of countably many formulas.

Fact 4.3. Let $G=G(\mathbb{M})$ be a type-definable group, and let $H$ be a subgroup of $G$ of bounded index that is type-definable over a small set of parameters $C$. Then $[G: H] \leq 2^{|T|+|C|}$. 
A fundamental fact about dependent groups is the absoluteness of their connected components:

Fact 4.4. [39] Let $T$ be dependent. Then $G_{A}^{00}=G_{\emptyset}^{00}$ for every small set A. In particular, the intersection of all subgroups of $G$ that are type-definable over a small set of parameters and of bounded index is a normal subgroup type-definable over $\emptyset$ and of index $\leq 2^{|T|}$.

This does not remain true for 2-dependent groups:

Example 4.5. Let $G$ be the group $\mathbb{F}_{2}^{(\omega)}$, where $\mathbb{F}_{2}$ is the finite field with 2 elements. Let $\mathcal{M}:=$ $\left(G, \mathbb{F}_{2}, 0,+, \cdot\right)$ be the structure with + the addition in $G$ and $\cdot$ the bilinear form $\left(a_{i}\right) \cdot\left(b_{i}\right)=\sum_{i} a_{i} b_{i}$ from $G^{2}$ to $\mathbb{F}_{2}$. Then $\operatorname{Th}(\mathcal{M})$ is simple and 2-dependent, and $G_{A}^{00}=\{g \in G: g \cdot a=0$ for all $a \in A\}$ (see [22, Section 3]), so the group $G_{A}^{00}$ gets smaller as $A$ grows.

However, in this example, for any small sets $A, B$, we have $G_{A B}^{00}=G_{A}^{00} \cap G_{B}^{00}$. The following theorem of Shelah shows that, up to a 'small' error, this holds in an arbitrary 2-dependent group:

Fact 4.6 [41]. Let $T$ be 2-dependent, $G=G(\mathbb{M})$ an A-type-definable group, $\kappa:=\beth_{2}(|A|+|T|)^{+}, \mathcal{M} \supseteq A$ a $\kappa$-saturated model, and $\bar{b}$ an arbitrary finite tuple in $\mathbb{M}$. Then

$$
G_{\mathcal{N} \bar{b}}^{00}=G_{\mathcal{M}}^{00} \cap G_{C \bar{b}}^{00}
$$

for some $C \subseteq \mathcal{M}$ with $|C|<\kappa$.

In this section, we generalize this result to $n$-dependent groups for arbitrary $n$. In order to state our generalization, we need to introduce an appropriate notion of independence.

Definition 4.7. ( $\kappa$-coheirs) For any cardinal $\kappa$, any model $\mathcal{M}$, and any tuple $a$, we write

$$
a \downarrow_{\mathcal{M}}^{u, \kappa} B
$$

if for any set $C \subset B \cup \mathcal{M}$ of size $<\kappa, \operatorname{tp}(a / C)$ is realized in $\mathcal{M}$.

When $\kappa=\aleph_{0}$, we get $a \perp_{\mathcal{M}}^{u, \aleph_{0}} B$ if and only if $\operatorname{tp}(a / B \mathcal{M})$ is finitely satisfiable in $\mathcal{M}$. In this case, we simply write $a \perp_{\mathcal{M}}^{u} B$ (the usual notation for coheir independence).

Recall that for an infinite cardinal $\kappa$ and $n \in \omega$, the cardinal $\beth_{n}(\kappa)$ is defined inductively by $\beth_{0}(\kappa)=\kappa$ and $\beth_{n+1}(\kappa)=2^{\beth_{n}(\kappa)}$. Then the Erdôs-Rado theorem says that $\left(\beth_{r}(\kappa)\right)^{+} \rightarrow\left(\kappa^{+}\right)_{\kappa}^{r+1}$ for all infinite $\kappa$ and $r \in \omega$.

Definition 4.8. (Generic position) Let $\mathcal{M}$ be a small model, $A$ a subset of $\mathcal{M}$ and $\bar{b}_{1}, \ldots, \bar{b}_{n-1}$ finite tuples in $\mathbb{M}$. We say that $\left(\mathcal{M}, A, \bar{b}_{1}, \ldots, \bar{b}_{n-1}\right)$ are in a generic position if there exist regular cardinals $\kappa_{1}<\kappa_{2}<\ldots<\kappa_{n-1}$ and models $\mathcal{M}_{0} \leq \mathcal{M}_{1} \leq \ldots \leq \mathcal{M}_{n-1}=\mathcal{M}$ such that $A \subseteq \mathcal{M}_{0}, \beth_{2}\left(\left|\mathcal{M}_{i}\right|\right)^{+} \leq \kappa_{i+1}$ for $i=0, \ldots, n-2$ and

$$
\bar{b}_{i} \perp_{\mathcal{M}_{i}}^{u, \kappa_{i}} \bar{b}_{<i} \mathcal{M}_{n-1}
$$

for all $1 \leq i \leq n-1$.

One general method to find elements in a generic position is given in Remark 4.14. But first let us state the main result of the section.

Theorem 4.9. Let $n \geq 1, T$ an $n$-dependent theory, $A \subseteq \mathbb{M} \models T$ a small parameter set and $G=G(\mathbb{M})$ a type-definable group in $T$ over $A$ be given. Let $\mathcal{M} \supseteq A$ be a small model and $\bar{b}_{1}, \ldots, \bar{b}_{n-1}$ finite tuples in $\mathbb{M}$ such that $\left(\mathcal{M}, A, \bar{b}_{1}, \ldots, \bar{b}_{n-1}\right)$ are in a generic position. 
Let $\left(H_{\alpha}: \alpha \in I\right)$ be any family of subgroups of $G$ of bounded index, with each $H_{\alpha}$ type-definable over $\mathcal{M}\left[\bar{b}_{1} \ldots \bar{b}_{n-1}\right.$. Then there is some $J \subseteq I$ with $|J| \leq \beth_{2}(|T|+|A|)$ such that

$$
\begin{aligned}
& \bigcap_{\alpha \in I} H_{\alpha} \cap \bigcap_{i=1, \ldots, n-1} G_{\mathcal{M} \cup \bar{b}_{1} \cup \ldots \cup \bar{b}_{i-1} \cup \bar{b}_{i+1} \cup \ldots \cup \bar{b}_{n-1}}^{00}= \\
& \bigcap_{\alpha \in J} H_{\alpha} \cap \bigcap_{i=1, \ldots, n-1} G_{\mathcal{M} \cup \bar{b}_{1} \cup \ldots \cup \bar{b}_{i-1} \cup \bar{b}_{i+1} \cup \ldots \cup \bar{b}_{n-1}}^{00} .
\end{aligned}
$$

Before we prove Theorem 4.9, let us observe the following corollary for the connected components of $n$-dependent groups.

Corollary 4.10. Let $T$ be a n-dependent theory, $A \subseteq \mathbb{M} \vDash T$ a small parameter set and $G=G(\mathbb{M}) a$ type-definable group in $T$ over $A$ be given. Let $\mathcal{M} \supseteq A$ be a small model and $\bar{b}_{1}, \ldots, \bar{b}_{n-1}$ finite tuples in $\mathbb{M}$ in a generic position. Then there is some $C \subseteq \mathcal{M}$ with $|C| \leq \beth_{2}(|T|+|A|)$ such that

$$
G_{\mathcal{M} \cup \bar{b}_{1} \cup \cdots \cup \bar{b}_{n-1}}^{00}=\bigcap_{i=1, \ldots, n-1} G_{\mathcal{M} \cup \bar{b}_{1} \cup \ldots \cup \bar{b}_{i-1} \cup \bar{b}_{i+1} \cup \ldots \cup \bar{b}_{n-1}}^{00} \cap G_{C \cup \bar{b}_{1} \cup \cdots \cup \bar{b}_{n-1}}^{00} .
$$

Proof. By Fact 4.2, we have

$$
\begin{gathered}
G_{\mathcal{M} \cup \bar{b}_{1} \cup \ldots \cup \bar{b}_{n-1}}^{00}= \\
\bigcap_{i=1, \ldots, n-1} G_{\mathcal{M} \cup \bar{b}_{1} \cup \ldots \cup \bar{b}_{i-1} \cup \bar{b}_{i+1} \cup \ldots \cup \bar{b}_{n-1}}^{00} \cap \bigcap\left\{G_{B \cup \bar{b}_{1} \cup \ldots \cup \bar{b}_{n-1}}^{00}: B \subseteq \mathcal{M} \text { countable }\right\} .
\end{gathered}
$$

By Theorem 4.9, it is already given by some sub-intersection of size at most $\beth_{2}(|T|+|A|)$. Letting $C \subseteq \mathcal{M}$ be the set containing all of the sets $B$ appearing in this sub-intersection gives the desired result.

Remark 4.11. Some variant of Corollary 4.10 is alluded to in [41, Discussion 2.14(2)], but we are not aware of any follow-up.

\section{Remark 4.12.}

1. For $n=1$, the assumptions of Corollary 4.10 are trivially satisfied by any sufficiently large model $\mathcal{M}=\mathcal{M}_{0}$, and the conclusion gives $G_{\mathcal{M}}^{00}=G_{C}^{00}$ for some small subset $C$ of $\mathcal{M}$ (since the first intersection on the right-hand side is over the empty set). This easily implies absoluteness of $G^{00}$.

2. For $n=2$, the assumption $\bar{b}_{1} \perp_{\mathcal{M}_{1}}^{u, \kappa_{1}} \mathcal{M}_{1}$ is clearly satisfied by any $\kappa_{1}$-saturated model $\mathcal{M}_{1} \supseteq A$ (taking $A \subseteq \mathcal{M}_{0} \leq \mathcal{M}_{1}$ arbitrary), and the conclusion gives $G_{\mathcal{M}_{1} \bar{b}_{1}}^{00}=G_{\mathcal{M}_{1}}^{00} \cap G_{\bar{b}_{1} C}^{00}$ - hence Fact 4.6 follows.

Problem 4.13. In order for our proof of Theorem 4.9 to work, we had to assume that the tuples $b_{1}, \ldots, b_{n-1}$ are in a generic position (which we think of intuitively as 'sufficiently independent from each other over $\mathcal{M}$ '). On the other hand, in the extreme opposite case when one of the tuples is in the algebraic closure of the other ones, the result holds trivially. Thus we ask if any hypothesis on the tuples $b_{1}, \ldots, b_{n-1}$ is needed for the result to hold.

The following remark provides a general method for finding tuples in a generic position.

Remark 4.14. Let $T$ be an arbitrary $\mathcal{L}$-theory, $A$ a small subset of $\mathbb{M}$ and $G=G(\mathbb{M})$ a group typedefinable over $A$. Let $T^{\prime}:=T^{\mathrm{Sk}}$ be a Skolemization of $T$ in a language $\mathcal{L}^{\prime} \supseteq \mathcal{L},\left|\mathcal{L}^{\prime}\right|=|\mathcal{L}|$. Let $\kappa_{0}:=|A|+|\mathcal{L}|$ and $\kappa_{i+1}:=\beth_{2}\left(\kappa_{i}\right)^{+}$, a regular cardinal, for $i=1, \ldots, n-2$. Next, choose mutually $\mathcal{L}^{\prime}(A)$-indiscernible sequences $J_{1}=\left(\bar{b}_{1, j}: j \in \kappa_{1}+1\right), \ldots, J_{n-1}=\left(\bar{b}_{n, j}: j \in \kappa_{n}+1\right)$ in some monster model $\mathbb{M}^{\prime}$ of $T^{\prime}$. Then for $i=1, \ldots, n-1$, let $I_{i}=\left(\bar{b}_{i, j}: j \in \kappa_{i}\right), \bar{b}_{i}=\bar{b}_{i, \kappa_{i}+1}$ (i.e. the last element in the sequence $\left.J_{i}\right)$, and $\mathcal{M}_{i}:=\operatorname{Sk}\left(A I_{1} \ldots I_{i}\right)$ (where $\mathrm{Sk}$ is the operation of taking the Skolem hull). Thus 
$A \subseteq \mathcal{M}_{0} \leq \mathcal{M}_{1} \leq \ldots \leq \mathcal{M}_{n-1}$ and $\left|\mathcal{M}_{i}\right|=\left|A \cup \bigcup_{1 \leq s \leq i} I_{s}\right|+\left|\mathcal{L}^{\prime}\right|=\kappa_{i}$; hence $\beth_{2}\left(\left|\mathcal{M}_{i}\right|\right)^{+} \leq \kappa_{i+1}$ holds for all $0 \leq i \leq n-2$.

We claim that $\bar{b}_{i} \perp_{\mathcal{M}_{i}}^{u, \kappa_{i}} \bar{b}_{<i} \mathcal{M}_{n-1}$ for every $1 \leq i \leq n-1$. To see this, fix $i$, and let $C \subseteq \mathcal{M}_{n-1}$ with $|C|<\kappa_{i}$ be arbitrary. By construction, there exist some $S_{i} \subseteq \kappa_{i}$ with $\left|S_{i}\right|<\kappa_{i}$ such that $C \subseteq \operatorname{dcl}_{\mathcal{L}^{\prime}}\left(A\left(b_{1, j}\right)_{j \in S_{1}} \ldots\left(b_{n-1, j}\right)_{j \in S_{n-1}}\right)$. As $\kappa_{i}>\left|S_{i}\right|$ is regular, there exists some $j^{*}>S_{i}$ in $\kappa_{i}$. But then by mutual $\mathcal{L}^{\prime}(A)$-indiscernibility of $J_{1}, \ldots, J_{n-1}$, we have that

$$
\bar{b}_{i, j^{*}} \equiv_{A\left(b_{1, j}\right)_{j \in S_{1}} \ldots\left(b_{n-1, j}\right)_{j \in S_{n-1}} \bar{b}_{<i}} \bar{b}_{i},
$$

hence $\bar{b}_{i, j^{*}} \equiv_{C \bar{b}_{<i}} \bar{b}_{i}$ and $\bar{b}_{i, j^{*}}$ is in $\mathcal{M}_{i}$.

Thus $\left(A, \mathcal{M}_{n-1}, \bar{b}_{1}, \ldots, \bar{b}_{n-1}\right)$ are in a generic position (both in the sense of $T$ and $\left.T^{\prime}\right)$.

\subsection{Proof of Theorem 4.9}

We are ready to prove the main theorem.

Proof (Proof of Theorem 4.9). Assume that the conclusion fails, and let $A \subseteq \mathcal{M}_{0} \leq \mathcal{M}_{1} \leq \ldots \leq$ $\mathcal{M}_{n-1}=\mathcal{M}$ witness the generic position as in Definition 4.8. Then, using Fact 4.2, we can find inductively a sequence of $\left(\mathcal{M}_{n-1} \cup \bar{b}_{1} \cup \cdots \cup \bar{b}_{n-1}\right)$-type-definable subgroups $H_{\alpha}, \alpha<\kappa:=\beth_{2}(|T|+|A|)^{+}$of $G$ of bounded index such that $H_{\alpha}=\bigcap_{m<\omega} \psi_{m}^{\alpha}\left(G ; \bar{c}_{\alpha}, \bar{b}_{1}, \ldots, \bar{b}_{n-1}\right)$ for some countable $\bar{c}_{\alpha}$ from $\mathcal{M}_{n-1}$, and elements $\left(d_{\alpha}\right)_{\alpha<\kappa}$ in $G$ such that

1. $d_{\alpha} \in \bigcap_{i=1, \ldots, n-1} G_{\mathcal{M}_{n-1} \cup \bar{b}_{1} \cup \ldots \cup \bar{b}_{i-1} \cup \bar{b}_{i+1} \cup \ldots \cup \bar{b}_{n-1}}^{00} \cap \bigcap_{\beta<\alpha} H_{\beta}$,

2. $d_{\alpha} \notin H_{\alpha}$.

Using compactness, and possibly replacing each $\psi_{m}^{\alpha}$ by a finite conjunction of $\psi_{i}^{\alpha}$, s, we may assume additionally that the following hold:

3. $\models \neg \psi_{0}^{\alpha}\left(d_{\alpha} ; \bar{c}_{\alpha}, \bar{b}_{1}, \ldots, \bar{b}_{n-1}\right)$,

4. For all $m \in \omega$, we have

$$
\begin{gathered}
\left\{\psi_{m+1}^{\alpha}\left(x ; \bar{c}_{\alpha}, \bar{b}_{1}, \ldots, \bar{b}_{n-1}\right), \psi_{m+1}^{\alpha}\left(y ; \bar{c}_{\alpha}, \bar{b}_{1}, \ldots, \bar{b}_{n-1}\right)\right\} \\
\psi_{m}^{\alpha}\left(x \cdot y ; \bar{c}_{\alpha}, \bar{b}_{1}, \ldots, \bar{b}_{n-1}\right) \wedge \psi_{m}^{\alpha}\left(x^{-1} ; \bar{c}_{\alpha}, \bar{b}_{1}, \ldots, \bar{b}_{n-1}\right) \wedge \\
\psi_{m}^{\alpha}\left(x \cdot y^{-1} ; \bar{c}_{\alpha}, \bar{b}_{1}, \ldots, \bar{b}_{n-1}\right)
\end{gathered}
$$

As there are only $|T|^{<\aleph_{0}}$ many formulas and $\operatorname{cf}(\kappa)>|T|^{\aleph_{0}}$, by the pigeonhole principle and after dropping some of the $H_{\alpha}$ 's, we can find $\left(\psi_{m}\right)_{m \in \omega}$ such that for all $\alpha<\kappa$,

$$
\psi_{m}^{\alpha}=\psi_{m}
$$

Claim 4.15. In addition to (1)-(4), we may assume that for all $i, j \in \omega$, we have that

$$
d_{i} \in H_{j} \Leftrightarrow i \neq j
$$

Proof. By Fact 4.3, for each $\beta<\kappa$, there is a partition $\left\{g_{\beta, \nu} H_{\beta}: v<\theta_{\beta}\right\}$ of $G$, where $\theta_{\beta}=[G$ : $\left.H_{\beta}\right] \leq 2^{\aleph_{0}}$. Therefore, considering $d_{\alpha}$ for some $\alpha<\kappa$, there is $v_{\alpha, \beta}<\theta_{\beta}$ such that

$$
d_{\alpha} \in g_{\beta, v_{\alpha, \beta}} H_{\beta} .
$$

As $\kappa=\beth_{2}(|T|+|A|)^{+}$by assumption, by Erdôs-Rado we can find an infinite subset $J$ of $\kappa$ and $v<2^{\aleph_{0}}$, such that for all $\alpha<\beta$ in $J$, we have that

$$
d_{\alpha} \in g_{\beta, \nu} H_{\beta}
$$


We may assume that $J=\omega$. Now let $i<j<m \in \omega$. Then by the above we have that

$$
d_{i} \in g_{m, v} H_{m} \text { and } d_{j} \in g_{m, v} H_{m},
$$

or in other words

$$
d_{i}=g_{m, v} h_{i} \text { and } d_{j}=g_{m, v} h_{j}
$$

for some $h_{i}, h_{j} \in H_{m}$. Thus

$$
d_{i}^{-1} d_{j}=h_{i}^{-1} g_{m, v}^{-1} g_{m, v} h_{j}=h_{i}^{-1} h_{j} \in H_{m}
$$

Now for $i \in \omega$, let

$$
e_{i}=d_{2 i}^{-1} d_{2 i+1}, \quad K_{i}=H_{2 i}, \quad \varphi_{m}=\psi_{m+1}, \text { and } \bar{f}_{i}=\bar{c}_{2 i} .
$$

Then, after replacing $d_{i}$ by $e_{i}, H_{i}$ by $K_{i}, \psi_{i}$ by $\varphi_{i}$, and $c_{i}$ by $f_{i},(1)$ and (4) are still satisfied. To show that condition (3) remains true, assume the opposite - that is, $\models \varphi_{0}\left(e_{i} ; \bar{f}_{i}, \bar{b}_{1}, \ldots, \bar{b}_{n-1}\right)$, which is equivalent to $\models \psi_{1}\left(e_{i} ; \bar{c}_{2 i}, \bar{b}_{1}, \ldots, \bar{b}_{n-1}\right)$. By (1), we know that $\models \psi_{1}\left(d_{2 i+1} ; \bar{c}_{2 i}, \bar{b}_{1}, \ldots, \bar{b}_{n-1}\right)$. Now, using that $d_{2 i}=d_{2 i+1} e_{i}^{-1}$ and (4), we can conclude that $\models \psi_{0}\left(d_{2 i} ; \bar{c}_{2 i}, \bar{b}_{1}, \ldots, \bar{b}_{n-1}\right)-$ contradicting (3) for the original sequence. Finally,

○ If $i \neq j$, then

$$
e_{i}=d_{2 i}^{-1} d_{2 i+1} \underset{\text { by }(*) \text { if } i<j}{\stackrel{\text { by }(1)}{\in} j<i} H_{2 j}=K_{j} .
$$

○ If $i=j$, then by (1) and (2), we have that $d_{2 i} \notin H_{2 i}$ but $d_{2 i+1} \in H_{2 i}$, so $e_{i} \notin H_{2 i}=K_{i}$. This also shows that condition (2) is still satisfied.

This finishes the proof of the claim.

Let $\overline{\mathbf{c}}:=\left(\bar{c}_{i}: i \in \omega\right)$.

Claim 4.16. There are sequences $\left(\bar{b}_{1, \gamma}: \gamma<\omega\right),\left(\bar{b}_{2, \gamma}: \gamma<\omega\right), \ldots,\left(\bar{b}_{n-1, \gamma}: \gamma<\omega\right)$ in $\mathcal{M}_{n-1}$ and elements $\left(d_{i, \gamma_{1}, \ldots, \gamma_{n-1}}:\left(i, \gamma_{1}, \ldots, \gamma_{n-1}\right) \in \omega^{n}\right)$ in $G$ such that

$$
\begin{aligned}
& d_{i, \gamma_{1}, \ldots, \gamma_{n-1}} \in H_{j, \delta_{1}, \ldots, \delta_{n-1}}:=\bigcap_{m<\omega} \psi_{m}\left(G ; \bar{c}_{j}, \bar{b}_{1, \delta_{1}}, \ldots, \bar{b}_{n-1, \delta_{n-1}}\right) \\
& \Leftrightarrow\left(i, \gamma_{1}, \ldots, \gamma_{n-1}\right) \neq\left(j, \delta_{1}, \ldots, \delta_{n-1}\right) \\
& \Leftrightarrow \vDash \psi_{n-1}\left(d_{i, \gamma_{1}, \ldots, \gamma_{n-1}} ; \bar{c}_{j}, \bar{b}_{1, \delta_{1}}, \ldots, \bar{b}_{n-1, \delta_{n-1}}\right) \text {. }
\end{aligned}
$$

Proof. We will prove the following claim by reverse induction on $l=1, \ldots, n$.

There are sequences $\left(\bar{b}_{l, \gamma}: \gamma<\omega\right),\left(\bar{b}_{l+1, \gamma}: \gamma<\omega\right), \ldots,\left(\bar{b}_{n-1, \gamma}: \gamma<\omega\right)$ in $\mathcal{M}_{n-1}$ and elements $\left(d_{i, \gamma_{l}, \ldots, \gamma_{n-1}}:\left(i, \gamma_{l}, \ldots, \gamma_{n-1}\right) \in \omega^{n-l+1}\right)$ in $G$ such that $\left(\dagger_{1}\right)-\left(\dagger_{4}\right)$ below hold:

$$
\begin{aligned}
& d_{i, \gamma_{l}, \ldots, \gamma_{n-1}} \in H_{j, \delta_{l}, \ldots, \delta_{n-1}}:=\bigcap_{m<\omega} \psi_{m}\left(G ; \bar{c}_{j}, \bar{b}_{1}, \ldots, \bar{b}_{l-1}, \bar{b}_{l, \delta_{l}}, \ldots, \bar{b}_{n-1, \delta_{n-1}}\right) \\
& \Leftrightarrow\left(i, \gamma_{l}, \ldots, \gamma_{n-1}\right) \neq\left(j, \delta_{l}, \ldots, \delta_{n-1}\right) \\
& \Leftrightarrow \vDash \psi_{n-l}\left(d_{i, \gamma_{l}, \ldots, \gamma_{n-1}} ; \bar{c}_{j}, \bar{b}_{1}, \ldots, \bar{b}_{l-1}, \bar{b}_{l, \delta_{l}}, \ldots, \bar{b}_{n-1, \delta_{n-1}}\right) \\
& \text { and } \\
& d_{i, \gamma_{l}, \ldots, \gamma_{n-1}} \in \bigcap_{\substack{t=1, \ldots, l-1 ; \\
\left(\delta_{l}, \ldots, \delta_{n-1}\right) \in \omega^{n-l}}} G_{\mathcal{M}_{l-1} \overline{\mathbf{c}} \bar{b}_{1} \ldots \bar{b}_{t-1} \bar{b}_{t+1} \ldots \bar{b}_{l-1} \bar{b}_{l, \delta_{l}} \ldots \bar{b}_{n-1, \delta_{n-1}}}
\end{aligned}
$$


for all $\gamma_{l}, \ldots, \gamma_{n-1}, \delta_{l}, \ldots, \delta_{n-1}, i, j \in \omega$ (where $\mathcal{M}_{0} \leq \cdots \leq \mathcal{M}_{n-1}$ are given by the assumption), and

$$
\left.\begin{array}{l}
\psi_{m+1}\left(x ; \bar{c}_{i}, \bar{b}_{1}, \ldots, \bar{b}_{l-1}, \bar{b}_{l, \gamma_{l}}, \ldots, \bar{b}_{n-1, \gamma_{n-1}}\right) \wedge \\
\psi_{m+1}\left(y ; \bar{c}_{i}, \bar{b}_{1}, \ldots, \bar{b}_{l-1}, \bar{b}_{l, \gamma_{l}}, \ldots, \bar{b}_{n-1, \gamma_{n-1}}\right) \\
\vdash \psi_{m}\left(x \cdot y ; \bar{c}_{i}, \bar{b}_{1}, \ldots, \bar{b}_{l-2}, \bar{b}_{l-1, \gamma_{l-1}}, \ldots, \bar{b}_{n-1, \gamma_{n-1}}\right) \wedge \\
\psi_{m}\left(x^{-1} ; \bar{c}_{i}, \bar{b}_{1}, \ldots, \bar{b}_{l-1}, \bar{b}_{l, \gamma_{l}}, \ldots, \bar{b}_{n-1, \gamma_{n-1}}\right) \wedge \\
\psi_{m}\left(x \cdot y^{-1} ; \bar{c}_{i}, \bar{b}_{1}, \ldots, \bar{b}_{l-1}, \bar{b}_{l, \gamma_{l}}, \ldots, \bar{b}_{n-1, \gamma_{n-1}}\right) \text { for all } i, m \in \omega .
\end{array}\right\}
$$

For $l=1$, this completes the proof of Claim 4.16.

For $l=n$, this is Claim 4.15 together with (1), (3) and (4).

Now suppose the claim is true for $1<l<n$, and we want to prove the claim for $l-1$. First, as $\bar{b}_{l-1} \perp_{\mathcal{M}_{l-1}}^{u, \kappa_{l-1}} \bar{b}_{<l-1} \mathcal{M}_{n-1}$, we can choose sequences $\left(\bar{b}_{l-1, \gamma}: \gamma<\kappa_{l-1}\right)$ in $\mathcal{M}_{l-1}$ and $\left(d_{i, \gamma_{l-1}, \ldots, \gamma_{n-1}}\right.$ : $\left.\left(i, \gamma_{l-1}, \ldots, \gamma_{n-1}\right) \in \omega \times \kappa_{l-1} \times \omega^{n-l}\right)$ in $G$ such that for any $\gamma<\kappa_{l-1}$ :

$$
\left.\begin{array}{l}
\left(\bar{b}_{l-1, \gamma}, d_{i, \gamma, \gamma_{l} \ldots, \gamma_{n-1}}:\left(i, \gamma_{l}, \ldots, \gamma_{n-1}\right) \in \omega^{n-l+1}\right) \text { has the same type as } \\
\left(\bar{b}_{l-1}, d_{i, \gamma_{l} \ldots, \gamma_{n-1}}:\left(i, \gamma_{l}, \ldots, \gamma_{n-1}\right) \in \omega^{n-l+1}\right) \text { over } \\
\mathcal{M}_{l-2}, \overline{\mathbf{c}}_{1} \bar{b}_{1}, \ldots, \bar{b}_{l-2} \cup\left\{b_{l-1, \delta}: \delta<\gamma\right\} \cup\left\{\bar{b}_{j, \delta}: j=l, \ldots, n-1, \delta<\omega\right\} .
\end{array}\right\}
$$

Then

5. By $\left(\dagger_{3}\right)$ for $t=l-1$ and $(\star)$, using that $\bar{b}_{l-1, \gamma} \in \mathcal{M}_{l-1}$ for all $\gamma<\kappa_{l-1}$, we obtain

$$
d_{i, \gamma_{l-1}, \ldots, \gamma_{n-1}} \in \bigcap\left\{H_{j, \delta_{l-1}, \ldots, \delta_{n-1}}:\left(j, \delta_{l}, \ldots, \delta_{n-1}\right) \in \omega^{n-l+1}, \delta_{l-1}<\gamma_{l-1}\right\}
$$

for all $i \in \omega$.

6. By $\left(\dagger_{1}\right)$ and $(\star)$

$$
d_{i, \gamma_{l-1}, \gamma_{l}, \ldots, \gamma_{n-1}} \in \bigcap\left\{H_{\left.j, \gamma_{l-1}, \delta_{l} \ldots, \delta_{n-1}\right)}:\left(i, \gamma_{l}, \ldots, \gamma_{n-1}\right) \neq\left(j, \delta_{l}, \ldots, \delta_{n-1}\right)\right\} .
$$

7. By $\left(\dagger_{1}\right)$ and $(\star)$

$$
d_{i, \gamma_{l-1}, \ldots, \gamma_{n-1}} \notin H_{i, \gamma_{l-1}, \ldots, \gamma_{n-1}},
$$

in particular by $\left(\dagger_{2}\right)$ and $(\star)$

$$
\vDash \neg \psi_{n-l}\left(d_{i, \gamma_{l-1}, \ldots, \gamma_{n-1}} ; \bar{c}_{i}, \bar{b}_{1}, \ldots, \bar{b}_{l-2}, \bar{b}_{l-1, \gamma_{l-1}}, \ldots, \bar{b}_{n-1, \gamma_{n-1}}\right) ;
$$

8. By $\left(\dagger_{4}\right)$ and $(\star)$ the formula

$$
\begin{gathered}
\psi_{m+1}\left(x ; \bar{c}_{i}, \bar{b}_{1}, \ldots, \bar{b}_{l-2}, \bar{b}_{l-1, \gamma_{l-1}}, \ldots, \bar{b}_{n-1, \gamma_{n-1}}\right) \wedge \\
\psi_{m+1}\left(y ; \bar{c}_{i}, \bar{b}_{1}, \ldots, \bar{b}_{l-2}, \bar{b}_{l-1, \gamma_{l-1}}, \ldots, \bar{b}_{n-1, \gamma_{n-1}}\right)
\end{gathered}
$$

implies the formula

$$
\begin{gathered}
\psi_{m}\left(x \cdot y ; \bar{c}_{i}, \bar{b}_{1}, \ldots, \bar{b}_{l-2}, \bar{b}_{l-1, \gamma_{l-1}}, \ldots, \bar{b}_{n-1, \gamma_{n-1}}\right) \wedge \\
\psi_{m}\left(x^{-1} ; \bar{c}_{i}, \bar{b}_{1}, \ldots, \bar{b}_{l-2}, \bar{b}_{l-1, \gamma_{l-1}}, \ldots, \bar{b}_{n-1, \gamma_{n-1}}\right) \wedge \\
\psi_{m}\left(x \cdot y^{-1} ; \bar{c}_{i}, \bar{b}_{1}, \ldots, \bar{b}_{l-2}, \bar{b}_{l-1, \gamma_{l-1}}, \ldots, \bar{b}_{n-1, \gamma_{n-1}}\right)
\end{gathered}
$$


9. By $\left(\dagger_{3}\right)$ and $(\star)$, and as $\bar{b}_{l-1, \delta_{l-1}}$ are all in $\mathcal{M}_{l-1}$,

$$
d_{i, \gamma_{l-1}, \ldots, \gamma_{n-1}} \in \bigcap_{\substack{t=1, \ldots, l-2 ; \\ \delta_{l-1} \leq \gamma_{l-1},\left(\delta_{l}, \ldots, \delta_{n-1}\right) \in \omega^{n-l}}} G_{\mathcal{M}_{l-2} \overline{\mathbf{c}} \bar{b}_{1} \ldots \bar{b}_{t-1} \bar{b}_{t+1} \ldots \bar{b}_{l-2} \bar{b}_{l-1, \delta_{l-1}} \ldots \bar{b}_{n-1, \delta_{n-1}}} .
$$

Consider the sequence of countable tuples

$$
\left(\bar{b}_{l-1, \gamma},\left(d_{i, \gamma, \gamma_{l} \ldots, \gamma_{n-1}}:\left(i, \gamma_{l}, \ldots, \gamma_{n-1}\right) \in \omega^{n-l+1}\right)\right)_{\gamma \in \kappa_{l-1}} .
$$

Note that for any $\delta<\kappa_{l-1}$, the group

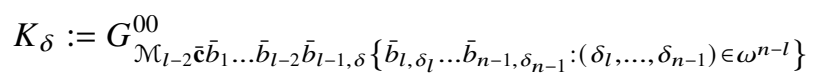

is type-definable over a set of size $\left|\mathcal{M}_{l-2}\right|+\boldsymbol{\aleph}_{0}=\left|\mathcal{M}_{l-2}\right|$ and has bounded index in $G$; hence by Fact 4.3, its index is at most $2^{\left|\mathcal{M}_{l-2}\right|}$. Let $\left(g_{\delta, v}: v<2^{\left|\mathcal{M}_{l-2}\right|}\right)$ be a set of representatives of its cosets in $G$. For each $\gamma<\delta<\kappa_{l-1}$, consider a countable tuple

$$
\bar{v}_{\gamma, \delta}:=\left(v_{i, \delta_{l}, \ldots, \delta_{n-1}}^{\gamma, \delta}: i, \delta_{l}, \ldots, \delta_{n-1} \in \omega\right)
$$

listing cosets of the elements $\left(d_{i, \gamma, \gamma_{l} \ldots, \gamma_{n-1}}:\left(i, \gamma_{l}, \ldots, \gamma_{n-1}\right) \in \omega^{n-l+1}\right)$ with respect to the group $K_{\delta}$. There are at most $\left(2^{\left|\mathcal{M}_{l-2}\right|}\right)^{\kappa_{0}}=2^{\left|\mathcal{M}_{l-2}\right|}$ possible choices for this tuple. As $\kappa_{l-1} \geq \beth_{2}\left(\left|\mathcal{M}_{l-2}\right|\right)^{+}$by assumption, applying Erdôs-Rado, there is an infinite subsequence such that $\bar{v}_{\gamma, \delta}$ is constant for all $\gamma<\delta$ from this subsequence. As in the proof of Claim 4.15, restricting to this subsequence, we have that (5)-(9) still hold, and for any fixed $\left(i, \gamma_{l}, \ldots, \gamma_{n-1}\right) \in \omega^{n-l+1}$, we have

$$
\begin{aligned}
& d_{i, \gamma^{\prime}, \gamma_{l}, \ldots, \gamma_{n-1}}^{-1} d_{i, \gamma, \gamma_{l} \ldots, \gamma_{n-1}} \in G_{\mathcal{M}_{l-2} \overline{\mathbf{c}} \bar{b}_{1} \ldots \bar{b}_{l-2} \bar{b}_{l-1, \delta}}^{00}\left\{\bar{b}_{l, \delta_{l}} \ldots \bar{b}_{n-1, \delta_{n-1}}:\left(\delta_{l}, \ldots, \delta_{n-1}\right) \in \omega^{n-l}\right\} \\
& \text { for all } \gamma<\gamma^{\prime}<\delta<\omega .
\end{aligned}
$$

Next, for $\left(i, \gamma_{l}, \ldots, \gamma_{n-1}\right) \in \omega^{n-l+1}$ and $\gamma_{l-1}<\kappa_{l-1}$, let

$$
\begin{aligned}
e_{i, \gamma_{l-1}, \ldots, \gamma_{n-1}} & =d_{i, 2 \gamma_{l-1}, \gamma_{l} \ldots, \gamma_{n-1}} d_{i, 2 \gamma_{l-1}+1, \gamma_{l} \ldots, \gamma_{n-1}}, \\
\bar{f}_{l-1, \gamma_{l-1}} & =\bar{b}_{l-1,2 \gamma_{l-1}}, \\
K_{i, \gamma_{l-1}, \gamma_{l} \ldots, \gamma_{n-1}} & =H_{i, 2 \gamma_{l-1}, \gamma_{l} \ldots, \gamma_{n-1}} .
\end{aligned}
$$

We will show that replacing

○ $d_{i, \gamma_{l-1}, \ldots, \gamma_{n-1}}$ by $e_{i, \gamma_{l-1}, \ldots, \gamma_{n-1}}, \bar{b}_{l-1, \gamma_{l-1}}$ by $\bar{f}_{l-1, \gamma_{l-1}}$,

$\circ H_{i, \gamma_{l-1}, \ldots, \gamma_{n-1}}$ by $K_{i, \gamma_{l-1}, \ldots, \gamma_{n-1}}$,

and restricting to the first countably many elements gives the desired sequences for $l-1$.

Note first that

$$
e_{i, \gamma_{l-1}, \ldots, \gamma_{n-1}} \in \bigcap_{\substack{t=1, \ldots, l-2 ; \\ \delta_{l-1} \leq \gamma_{l-1},\left(\delta_{l}, \ldots, \delta_{n-1}\right) \in \omega^{n-l}}} G_{\mathcal{M}_{l-2} \overline{\mathbf{c}} \bar{b}_{1} \ldots \bar{b}_{t-1} \bar{b}_{t+1} \ldots \bar{b}_{l-2} \bar{b}_{l-1,2 \delta_{l-1}}, \bar{b}_{l, \delta_{l}} \ldots \bar{b}_{n-1, \delta_{n-1}}}
$$

by (9).

Now let $\left(j, \gamma_{l-1}, \ldots, \gamma_{n-1}\right) \neq\left(i, \delta_{l-1}, \ldots, \delta_{n-1}\right)$. We consider two cases:

Case 1: $\gamma_{l-1}=\delta_{l-1}$.

In this case, $\left(j, \gamma_{l}, \ldots, \gamma_{n-1}\right) \neq\left(i, \delta_{l}, \ldots, \delta_{n-1}\right)$. Thus

$$
d_{i, 2 \gamma_{l-1}, \gamma_{l}, \ldots, \gamma_{n-1}} \in H_{j, 2 \gamma_{l-1}, \delta_{l}, \ldots, \delta_{n-1}} \text { by }(6)
$$


and

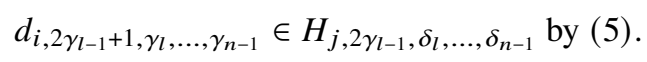

So

10. $e_{i, \gamma_{l-1}, \ldots, \gamma_{n-1}}=d_{i, 2 \gamma_{l-1}, \gamma_{l}, \ldots, \gamma_{n-1}}^{-1} d_{i, 2 \gamma_{l-1}+1, \gamma_{l} \ldots, \gamma_{n-1}} \in H_{j, 2 \gamma_{l-1}, \delta_{l}, \ldots, \delta_{n-1}}=K_{j, \delta_{l-1}, \delta_{l}, \ldots, \delta_{n-1}}$, and in particular

11. $\vDash \psi_{n-l+1}\left(e_{i, \gamma_{l-1}, \ldots, \gamma_{n-1}} ; \bar{c}_{j}, \bar{f}_{l-1, \delta_{l-1}}, b_{l, \delta_{l}} \ldots, \bar{b}_{n-1, \delta_{n-1}}\right)$.

Case 2: $\gamma_{l-1} \neq \delta_{l-1}$.

If $\delta_{l-1}<\gamma_{l-1}$, then $e_{i, \gamma_{l-1}, \ldots, \gamma_{n-1}}=d_{i, 2 \gamma_{l-1}, \gamma_{l}, \ldots, \gamma_{n-1}}^{-1} d_{i, 2 \gamma_{l-1}+1, \gamma_{l} \ldots, \gamma_{n-1}} \in H_{j, 2 \delta_{l-1}, \delta_{l}, \ldots, \delta_{n-1}}$ by (5). So without loss of generality $\gamma_{l-1}<\delta_{l-1}$. Then (††) implies in particular that

$$
e_{i, \gamma_{l-1}, \ldots, \gamma_{n-1}}=d_{i, 2 \gamma_{l-1}+1, \gamma_{l}, \ldots, \gamma_{n-1}}^{-1} d_{i, 2 \gamma_{l-1}, \gamma_{l} \ldots, \gamma_{n-1}} \in H_{j, 2 \delta_{l-1}, \delta_{l}, \ldots, \delta_{n-1}}
$$

and

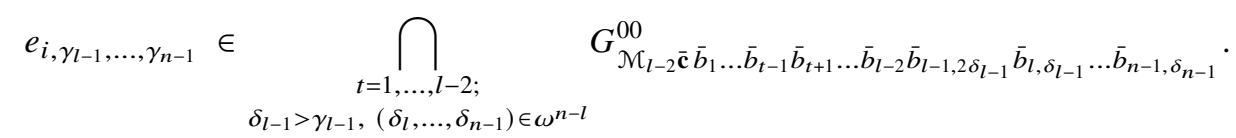

Together with $(* *)$, this gives

12. $e_{i, \gamma_{l-1}, \ldots, \gamma_{n-1}} \in \bigcap_{\left(\delta_{l-1}, \ldots, \delta_{n-1}\right) \in \omega \times \omega^{n-l}} G_{\mathcal{M}_{l-2} \overline{\mathbf{c}} \bar{b}_{1} \ldots \bar{b}_{t-1} \bar{b}_{t+1} \ldots \bar{b}_{l-2} \bar{f}_{l-1, \delta_{l-1}} \bar{b}_{l, \delta_{l-1}} \ldots \bar{b}_{n-1, \delta_{n-1}}}$.

On the other hand, for given $\left(j, \gamma_{l-1}, \ldots, \gamma_{n-1}\right) \in \omega^{n-l+2}$, by (7) we have

$$
d_{i, 2 \gamma_{l-1}, \gamma_{l} \ldots, \gamma_{n-1}} \notin H_{i, 2 \gamma_{l-1}, \gamma_{l} \ldots, \gamma_{n-1}},
$$

and by (5) we have

$$
d_{i, 2 \gamma_{l}+1, \gamma_{l} \ldots, \gamma_{n-1}} \in H_{i, 2 \gamma_{l-1}, \gamma_{l}, \ldots, \gamma_{n-1}} .
$$

Hence

13. $e_{i, \gamma_{l-1}, \ldots, \gamma_{n-1}}=d_{i, 2 \gamma_{l-1}, \gamma_{l} \ldots, \gamma_{n-1}}^{-1} d_{i, 2 \gamma_{l-1}+1, \gamma_{l} \ldots, \gamma_{n-1}} \notin H_{i, 2 \gamma_{l-1}, \gamma_{l} \ldots, \gamma_{n-1}}=K_{i, \gamma_{l-1}, \ldots, \gamma_{n-1}}$.

Suppose toward a contradiction that

$$
\vDash \psi_{n-l+1}\left(e_{i, \gamma_{l-1}, \ldots, \gamma_{n-1}} ; \bar{c}_{i}, \bar{f}_{l-1, \gamma_{l-1}}, b_{l, \gamma_{l}} \ldots, \bar{b}_{n-1, \gamma_{n-1}}\right) .
$$

By (5), we also have that

$$
\vDash \psi_{n-l+1}\left(d_{i, 2 \gamma_{l-1}+1, \gamma_{l}, \ldots, \gamma_{n-1}+1} ; \bar{c}_{i}, \bar{f}_{l-1, \gamma_{l-1}}, \bar{b}_{l, \gamma_{l}} \ldots, \bar{b}_{n-1, \gamma_{n-1}}\right) .
$$

Since $d_{i, 2 \gamma_{l-1}, \ldots, \gamma_{n-1}}=d_{i, 2 \gamma_{l-1}+1, \gamma_{l} \ldots, \gamma_{n-1}} e_{i, \gamma_{l-1}, \ldots, \gamma_{n-1}}^{-1}$, using (8), we conclude that

$$
\begin{gathered}
\models \psi_{n-l}\left(d_{i, 2 \gamma_{l-1}, \gamma_{l} \ldots, \gamma_{n-1}} ; \bar{c}_{i}, \bar{f}_{l-1, \gamma_{l-1}}, \bar{b}_{l, \gamma_{l}} \ldots, \bar{b}_{n-1, \gamma_{n-1}}\right) \\
=\psi_{n-l}\left(d_{i, 2 \gamma_{l-1}, \gamma_{l} \ldots, \gamma_{n-1}} ; \bar{c}_{i}, \bar{b}_{l-1,2 \gamma_{l-1}}, \ldots, \bar{b}_{n-1, \gamma_{n-1}}\right),
\end{gathered}
$$

contradicting (7). Thus

14. $\not \psi_{n-l+1}\left(e_{i, \gamma_{l-1}, \ldots, \gamma_{n-1}} ; \bar{c}_{i}, \bar{f}_{l-1, \gamma_{l-1}}, b_{l, \gamma_{l}} \ldots, \bar{b}_{n-1, \gamma_{n-1}}\right)$.

Now, for $l-1$, we obtain $\left(\dagger_{1}\right)$ from (10) and (13), (†2) from (11) and (14), (†3) from (12), and (†4 $)$ from (8) by replacing $e_{i, \gamma_{l-1}, \ldots, \gamma_{n-1}}$ by $d_{i, \gamma_{l-1}, \ldots, \gamma_{n-1},}, \bar{f}_{l-1, \gamma_{l-1}}$ by $\bar{b}_{l-1, \gamma_{l-1}}$, and $K_{i, \gamma_{l-1}, \ldots, \gamma_{n-1}}$ by $H_{i, \gamma_{l-1}, \ldots, \gamma_{n-1}}$. This finishes the proof of the claim. 
Claim 4.17. For any $I \subset(\omega)^{n}$, there is some $d \in G$ such that

$$
\vDash \psi_{n+1}\left(d ; \bar{c}_{i}, b_{1, \gamma_{1}}, \ldots, \bar{b}_{n-1, \gamma_{n-1}}\right)
$$

if and only if $\left(i, \gamma_{1}, \ldots, \gamma_{n-1}\right) \notin I$.

Proof. Suppose first that $I$ is finite, and let $\left(\left(i_{l}, \gamma_{1, l}, \ldots, \gamma_{n-1, l}\right): l \leq s\right)$ be an enumeration of the elements in $I$. Define $d=d_{i_{0}, \gamma_{1,0}, \ldots, \gamma_{n-1,0}} \cdot \ldots \cdot d_{i_{s}, \gamma_{1, s}, \ldots, \gamma_{n-1, s}}$.

If $\left(j, \delta_{1}, \ldots, \delta_{n-1}\right) \notin I$, then $d_{i_{l}, \gamma_{1, l}, \ldots, \gamma_{n-1, l}} \in H_{j, \delta_{1}, \ldots, \delta_{n-1}}$ for all $l \leq s$ by Claim 4.16, and hence $d \in H_{j, \delta_{1}, \ldots, \delta_{n-1}}$ and in particular $\models \psi_{n+1}\left(d ; \bar{c}_{j}, \bar{b}_{1, \delta_{1}}, \ldots, \bar{b}_{n-1, \delta_{n-1}}\right)$.

On the other hand, consider $\left(i_{l}, \gamma_{1, l}, \ldots, \gamma_{n-1, l}\right) \in I$. Let

$$
e_{1}=d_{i_{0}, \gamma_{1,0}, \ldots, \gamma_{n-1,0}} \cdot \ldots \cdot d_{i_{l-1}, \gamma_{1, l-1}, \ldots, \gamma_{n-1, l-1}}
$$

and

$$
e_{2}=d_{i_{l}, \gamma_{1, l+1}, \ldots, \gamma_{n-1, l+1}} \cdot \ldots \cdot d_{i_{s}, \gamma_{1, s}, \ldots, \gamma_{n-1, s}} .
$$

Then $d_{i_{l}, \gamma_{1, l}, \ldots, \gamma_{n-1, l}}=e_{1}^{-1} d e_{2}^{-1}$. Observe that

$$
\begin{gathered}
\vDash \neg \psi_{n-1}\left(d_{i_{l}, \gamma_{1, l}, \ldots, \gamma_{n-1, l}}, \bar{c}_{i_{l}}, b_{1, \gamma_{1, l}}, \ldots, \bar{b}_{n-1, \gamma_{n-1, l}}\right), \\
\vDash \psi_{n}\left(e_{2}^{-1}, \bar{c}_{i_{l}}, b_{1, \gamma_{1, l}}, \ldots, \bar{b}_{n-1, \gamma_{n-1, l}}\right) \text { and } \\
=\psi_{n+1}\left(e_{1}^{-1}, \bar{c}_{i_{l}}, b_{1, \gamma_{1, l}}, \ldots, \bar{b}_{n-1, \gamma_{n-1, l}}\right)
\end{gathered}
$$

by Claim 4.16. Using (8), we conclude that

$$
\begin{aligned}
& \vDash \neg \psi_{n-1}\left(d_{i_{l}, \gamma_{1, l}, \ldots, \gamma_{n-1, l}}, \bar{c}_{i_{l}}, b_{1, \gamma_{1, l}}, \ldots, \bar{b}_{n-1, \gamma_{n-1, l}}\right) \\
\rightarrow & \vDash \neg \psi_{n}\left(e_{1}^{-1} d, \bar{c}_{i_{l}}, b_{1, \gamma_{1, l}}, \ldots, \bar{b}_{n-1, \gamma_{n-1, l}}\right) \\
\rightarrow & \vDash \neg \psi_{n+1}\left(d, \bar{c}_{i_{l}}, b_{1, \gamma_{1, l}}, \ldots, \bar{b}_{n-1, \gamma_{n-1, l}}\right) .
\end{aligned}
$$

The claim follows by compactness.

Finally, Claim 4.17 contradicts $n$-dependence of $\psi_{n+1}$, which finishes the proof.

\section{2-dependence for compositions of dependent relations and binary functions}

The aim of this section is prove the following Composition Lemma (Theorem 5.12 below): a composition of a relation (of any arity) definable in a model of a dependent theory with arbitrary binary functions is 2-dependent. This result is crucial in our proof of 2-dependence of non-degenerate bilinear forms over dependent fields in Section 6. Toward this purpose, we first develop a general type-counting criterion for 2-dependent theories in Section 5.1 and then apply it along with the set-theoretic absoluteness to deduce the Composition Lemma in Section 5.2.

\subsection{Characterization of 2-dependence by a type-counting criterion}

We first recall a type-counting criterion characterizing dependent theories. For the following two facts; see for example [16] or [13, Section 6] and references there. We recall that for an infinite $\kappa$, ded $\kappa$ is the supremum of the number of Dedekind cuts among all linear orders of cardinality $\kappa$.

Fact 5.1 (Shelah). Let $T$ be a theory in a countable language, and for an infinite cardinal $\kappa$, let $f_{T}(\kappa):=\sup \left\{\left|S_{1}(M)\right|:|M|=\kappa, M \models T\right\}$.

1. If $T$ is dependent, then $f_{T}(\kappa) \leq(\operatorname{ded} \kappa)^{\aleph_{0}}$ for all infinite cardinals $\kappa$.

2. If $T$ is not dependent, then $f_{T}(\kappa)=2^{\kappa}$ for all infinite cardinals $\kappa$. 
In a model of ZFC satisfying the Generalized Continuum Hypothesis, ded $\kappa=2^{\kappa}$ for all infinite cardinals $\kappa$. However, there are models of ZFC in which these two functions are different:

Fact 5.2 (Mitchell [35]). For every cardinal $\kappa$ of uncountable cofinality, there exists a cardinal preserving Cohen extension such that $(\operatorname{ded} \kappa)^{\aleph_{0}}<2^{\kappa}$.

The combination of Facts 5.1 and 5.2 tells us that it is possible to detect whether a theory is dependent by counting types using that dependence of a formula is a set-theoretically absolute property.

We want to provide a formula-free characterization of $n$-dependence of a theory that does not include any assumption of indiscernibility of the witnessing sequence over the additional parameters (unlike the characterization in Proposition 2.9, where additional indiscernibility over the parameter needs to be assumed). We achieve it here for 2-dependence by providing an analog of Fact 5.1 in this case (which characterizes 2-dependence of a theory when working in a model of ZFC with $\operatorname{ded}(\kappa)<2^{\kappa}$ for some cardinal $\kappa \geq|T|)$.

Given a set $X$ and a family $\mathcal{F}$ of subsets of $X$ and $Y \subseteq X$, one says that $Y$ is shattered by $\mathcal{F}$ if for every $Z \subseteq Y$, there exists some $S \in \mathcal{F}$ such that $Z=Y \cap S$. In what follows, $T$ is a complete theory in a language $\mathcal{L}$, and we work in a monster model $\mathbb{M} \vDash T$.

Lemma 5.3. Let $\varphi\left(x ; y_{1}, y_{2}\right) \in \mathcal{L}$ be 2-dependent. Then there is some $n \in \mathbb{N}$ such that for any $c \in \mathbb{M}_{x}$, any $I \subseteq \mathbb{M}_{y_{1}}, J \subseteq \mathbb{M}_{y_{2}}$ endless mutually indiscernible sequences, and any $A \subseteq I$ of size $>n$, there is some $b_{A} \in J$ such that $A$ cannot be shattered by the family $\left\{\varphi\left(c, y_{1}, b\right): b \in J, b>b_{A}\right\}$.

Proof. Assume that $I, J$ are endless mutually indiscernible sequences and $c$ is such that the conclusion is not satisfied for any $n \in \omega$. Let $D \subseteq I \times J$ be any finite set. Let $a_{1}<\ldots<a_{n}$ and $b_{1}<\ldots<b_{m}$ list the projections of $D$ on $I$ and $J$, respectively. By assumption, there is some $A \subseteq I$ of size $n$ such that for any $b^{\prime} \in J, A$ is shattered by the family $\left\{\varphi\left(c, y_{1}, b\right): b \in J, b>b^{\prime}\right\}$. List $A$ as $a_{1}^{\prime}<\ldots<a_{n}^{\prime}$. Then we can choose some $b_{1}^{\prime}<\ldots<b_{m}^{\prime} \in J$ such that $\models \varphi\left(c, a_{i}^{\prime}, b_{j}^{\prime}\right) \Longleftrightarrow\left(a_{i}, b_{j}\right) \in D$. As $I, J$ are mutually indiscernible, taking an automorphism of $\mathbb{M}$ sending $a_{i}^{\prime}$ to $a_{i}$ and $b_{j}^{\prime}$ to $b_{j}$, for all $1 \leq i \leq n, 1 \leq j \leq m$, $c$ is sent to some $c_{D}$ such that $\models \varphi\left(c_{D}, a_{i}, b_{j}\right) \Longleftrightarrow\left(a_{i}, b_{j}\right) \in D$. This implies that $\varphi\left(x ; y_{1}, y_{2}\right)$ is not 2-dependent, a contradiction. Hence the conclusion holds for $c, I, J$ for some $n$.

By compactness, we conclude that $n$ can be chosen depending only on $\varphi$ (and not on $I, J, c$ ).

We will need the following fact (originally due to Shelah, with simplifications by Adler and Casanovas; see e.g. [10, Lemma 2.7.1]).

Fact 5.4. If $\kappa$ is an infinite cardinal, $\mathcal{F} \subseteq 2^{\kappa}$ and $|\mathcal{F}|>\operatorname{ded} \kappa$, then for each $n \in \omega$, there is some $S \subseteq \kappa$ such that $|S|=n$ and $\mathcal{F} \uparrow S=2^{S}$.

Definition 5.5. Given sets $B \subseteq \mathbb{M}_{x}, A \subseteq \mathbb{M}_{y}$ and a formula $\varphi(x, y) \in \mathcal{L}$, we denote by $S_{\varphi, B}(A)$ the set of all $\varphi$-types over $A$ realized in $B$, where by a $\varphi$-type over $A$, we mean a maximal consistent collection of formulas of the form $\varphi(x, a), \neg \varphi(x, a)$ with $a \in A$. And $S_{B}(A)$ denotes the set of all complete types over $A$ realized in $B$.

Proposition 5.6. Let $T$ be 2-dependent, let $\kappa \geq|T|$ be an infinite cardinal, and let $\lambda>\kappa$ be a regular cardinal. Then for any mutually indiscernible sequences $I=\left(a_{i}: i \in \kappa\right), J=\left(b_{j}: j \in \lambda\right)$ of finite tuples and a finite tuple $c$, there is some $\beta \in \lambda$ such that

$$
\left|S_{J_{>\beta}}(I \times\{c\})\right| \leq(\operatorname{ded} \kappa)^{|T|} .
$$

Proof. Let $I, J$ and $c$ be given. We will show that for each $\varphi\left(x ; y_{1}, y_{2}\right) \in \mathcal{L}$, there is some $\beta_{\varphi} \in \lambda$ such that $\left|S_{\varphi, J_{>\beta} \varphi}(I \times\{c\})\right| \leq \operatorname{ded} \kappa$. This is enough, as then we can take any $\beta \in \lambda$ with $\beta>\beta_{\varphi}$ for all $\varphi \in \mathcal{L}$ (possible as $\lambda=\operatorname{cof}(\lambda)>|T|)$, and $\left|S_{J_{>\beta}}(I \times\{c\})\right| \leq\left|\prod_{\varphi \in \mathcal{L}} S_{\varphi, J_{>\beta}}(I \times\{c\})\right| \leq(\operatorname{ded} \kappa)^{|T|}$. 
So let $\varphi\left(x ; y_{1}, y_{2}\right) \in \mathcal{L}$ be fixed, and assume that for any $\beta \in \lambda$,

$$
\left|S_{\varphi, J_{>\beta}}(I \times\{c\})\right|>\operatorname{ded} \kappa .
$$

Then by Fact 5.4, considering $\mathcal{F}=\left\{f_{p}: p \in S_{\varphi, J_{>\beta}}(I \times\{c\})\right\}$ (where $f_{p} \in 2^{\kappa}$ is given by $f_{p}(\alpha)=$ $1 \Longleftrightarrow \varphi\left(x ; a_{\alpha}, c\right) \in p$, for all $\left.\alpha \in \kappa\right)$, for any $n \in \omega$, there is some $S \subseteq I,|S|=n$ such that $S$ is shattered by the family $\left\{\varphi\left(b_{j} ; y_{1}, c\right): j \in \lambda, j>\beta\right\}$. Using regularity of $\lambda$, by transfinite induction we can choose a strictly increasing sequence $\left(\beta_{\alpha}: \alpha \in \lambda\right)$ with $\beta_{\alpha} \in \lambda$ such that for each $\alpha \in \lambda$, there is some $S_{\alpha} \subseteq I,\left|S_{\alpha}\right|=n$ shattered by the family $\left\{\varphi\left(b_{j} ; y_{1}, c\right): j \in \lambda, \beta_{\alpha}<j<\beta_{\alpha+1}\right\}$. As $\lambda>\kappa=\kappa^{n}$ is regular, passing to a subsequence, we may assume that there is some $S \subseteq I,|S|=n$ such that $S_{\alpha}=S$ for all $\alpha \in \lambda$ - that is, this set $S$ can be shattered arbitrarily far into the sequence. Now, by Lemma 5.3, this contradicts 2 -dependence of $\varphi$ if we take $n$ large enough.

Lemma 5.7. For any cardinal $\kappa$ and any regular cardinal $\lambda \geq 2^{\kappa}$, there is a bipartite graph $\mathcal{G}_{\kappa, \lambda}=$ $(\kappa, \lambda, E)$ (where its parts are identified with the cardinals $\kappa$ and $\lambda$, and $E \subseteq \kappa \times \lambda$ is the edge relation) satisfying the following: for any sets $A, A^{\prime} \subseteq \kappa$ with $A \cap A^{\prime}=\emptyset$ and any $b \in \lambda$, there is some $b^{*} \in \lambda$, $b^{*}>b$ satisfying $\bigwedge_{a \in A} E\left(a, b^{*}\right) \wedge \bigwedge_{a^{\prime} \in A^{\prime}} E\left(a^{\prime}, b^{*}\right)$.

Proof. Let $\lambda \geq 2^{\kappa}$ be any regular cardinal. Let

$$
D:=\left\{\left(A, A^{\prime}, b\right): A, A^{\prime} \subseteq \kappa, A \cap A^{\prime}=\emptyset, b \in \lambda\right\} .
$$

Then $|D| \leq \lambda$ by assumption; we enumerate it as $\left(\left(A_{\alpha}, A_{\alpha}^{\prime}, b_{\alpha}\right): \alpha<\lambda\right)$. We define $E_{\alpha} \subseteq \kappa \times \lambda$ by transfinite induction on $\alpha<\lambda$. On step $\alpha$, we choose some $c_{\alpha} \in \lambda$ such that $c_{\alpha}>\left\{b_{\beta}, c_{\beta}: \beta<\alpha\right\}-$ possible by regularity of $\lambda-$ and we take $E_{\alpha}:=\left\{\left(a, c_{\alpha}\right): a \in A_{\alpha}\right\}$. Let $E:=\bigsqcup_{\alpha<\lambda} E_{\alpha}-$ it satisfies the requirement by construction.

Proposition 5.8. Assume that $\varphi(x, y, z)$ is not 2-dependent. Then for every regular $\lambda>2^{\kappa}$, there exist mutually indiscernible sequences $I=\left(a_{i}: i<\kappa\right)$ in $\mathbb{M}_{y},\left(b_{j}: j<\lambda\right)$ in $\mathbb{M}_{x}$ and $c \in \mathbb{M}_{z}$ such that for every $\beta<\lambda$, we have $\left|S_{\varphi, J_{>\beta}}(I \times\{c\})\right|=2^{\kappa}$.

Proof. By assumption, for any $\kappa, \lambda$, we can find some mutually indiscernible sequences $I, J$ such that the family $\left\{\varphi(x, y, c): c \in \mathbb{M}_{z}\right\}$ shatters $I \times J$. In particular, we can find $c$ such that $\mathbb{M} \vDash \varphi\left(b_{j}, a_{i}, c\right) \Longleftrightarrow$ $\mathcal{G}_{\kappa, \lambda} \vDash E\left(a_{i}, b_{j}\right)$, and we can conclude by Lemma 5.7.

Propositions 5.6 and 5.8 together provide an analog of Fact 5.1 for 2-dependent theories that will be used in the proof of the Composition Lemma in Section 5.1. We conclude this subsection with a brief discussion of some questions arising in connection to this criterion (and not used in the rest of the paper).

Definition 5.9. We say that a theory $T$ is globally 2-dependent if there are a cardinal $\kappa$ and a regular cardinal $\lambda \geq 2^{\kappa}$ such that for $c$ and $\mathcal{G}_{\kappa, \lambda}$ given by Lemma 5.7, the following holds: for any mutually indiscernible sequences $I=\left(a_{i}: i \in \kappa\right), J=\left(b_{j}: j \in \lambda\right)$ of finite tuples there are $i \in \kappa$ and $j, j^{\prime} \in \lambda$ such that $c a_{i} b_{j} \equiv c a_{i} b_{j^{\prime}}$ but $E(i, j) \wedge \neg E\left(i, j^{\prime}\right)$.

So the idea is that $T$ is globally 2-dependent if on mutually indiscernible sequences we cannot distinguish the edges from the non-edges of a random graph by a complete type (as opposed to witnessing the edges with realizations of a single formula). We have the following connection between 2-dependence and global 2-dependence.

\section{Proposition 5.10.}

1. If $T$ is globally 2-dependent, then it is 2-dependent.

2. Let $T$ be a countable 2-dependent theory, and assume that there exists a cardinal $\kappa$ such that $(\operatorname{ded} \kappa)^{\kappa_{0}}<2^{\kappa}$. Then $T$ is globally 2-dependent. 
Proof.

(1) If $\varphi\left(x, y_{1}, y_{2}\right)$ is a formula witnessing failure of 2-dependence, then the proof of Proposition 5.8 shows that $T$ is not globally 2 -dependent.

(2) Fix $\kappa$, and let $\lambda$ be any regular cardinal $\geq 2^{\kappa}$. Let $\mathcal{G}_{\kappa, \lambda}$ be as given by Lemma 5.7. Moreover, let $I, J$ and $c$ be as in Definition 5.9. By Proposition 5.6, there is some $\beta \in \lambda$ such that $\left|S_{J_{>\beta}}(I)\right| \leq(\operatorname{ded} \kappa)^{\aleph_{0}}$. On the other hand, by definition of $\mathcal{G}_{\kappa, \lambda}$, we still have $\left|S_{E,\{\alpha \in \lambda: \alpha>\beta\}}(\kappa)\right|=2^{\kappa}>(\operatorname{ded} \kappa)^{\aleph_{0}}$ by assumption. Then we can find some $j, j^{\prime} \in \lambda$ such that $\operatorname{tp}_{E}(j / \kappa) \neq \operatorname{tp}_{E}\left(j^{\prime} / \kappa\right)$ but tp $\left(b_{j} / I c\right)=\operatorname{tp}\left(b_{j^{\prime}} / I c\right)$. In other words, there is some $i \in \kappa$ such that $E(i, j) \leftrightarrow \neg E\left(i, j^{\prime}\right)$ and still $b_{j} a_{i} c \equiv b_{j^{\prime}} a_{i} c$, as wanted.

Problem 5.11. Is there an analogous type-counting criterion for $n$-dependence, $n \geq 3$ ? Is it true that $n$-dependence implies global n-dependence (defined analogously), in ZFC, or at least consistently for $n>2$ ?

Concerning this problem, we remark that at least $n$-dependence implies global $n$-dependence when $T$ is $\boldsymbol{\aleph}_{0}$-categorical (since every type in finitely many variables is equivalent to a formula).

\subsection{The Composition Lemma}

All of the variables below are allowed to be tuples of arbitrary finite length.

Theorem 5.12 (Composition Lemma). Let $\mathcal{M}_{0}$ be a dependent structure in a language $\mathcal{L}_{0}$, and let $\mathcal{M}$ be an arbitrary expansion of $\mathcal{M}$ in some language $\mathcal{L} \supseteq \mathcal{L}_{0}$. Let $\varphi\left(x_{1}, \ldots, x_{d}\right)$ be an $\mathcal{L}_{0}$-formula. For each $i \in\{1, \ldots, d\}$, fix some $s_{i}<t_{i} \in\{1,2,3\}$ and let $f_{i}: M_{y_{s_{i}}} \times M_{y_{t_{i}}} \rightarrow M_{x_{i}}$ be an L-definable binary function. Then the $\mathcal{L}$-formula

$$
\psi\left(y_{1} ; y_{2}, y_{3}\right):=\varphi\left(f_{1}\left(y_{s_{1}}, y_{t_{1}}\right), \ldots, f_{d}\left(y_{s_{d}}, y_{t_{d}}\right)\right)
$$

is 2-dependent (with respect to $\mathrm{Th}_{\mathcal{L}}(\mathcal{M})$ ).

Proof. We work in a monster model $\mathbb{M}$ of $T:=\operatorname{Th}_{\mathcal{L}}(\mathcal{M})$. Note that then the $\mathcal{L}_{0}$-reduct $\mathbb{M}_{0}$ of $\mathbb{M}$ is a monster model of $T_{0}:=\mathrm{Th}_{\mathcal{L}_{0}}\left(\mathcal{M}_{0}\right)$, and $T_{0}$ is dependent. Assume that the formula $\psi\left(y_{1} ; y_{2}, y_{3}\right)$ as in the statement of the theorem is not 2-dependent. Let $I=\left(a_{\alpha}: \alpha<\kappa\right)$ with $a_{\alpha} \in M_{y_{2}}, J=\left(b_{\beta}: \beta<\lambda\right)$ with $b_{\beta} \in M_{y_{1}}$ and $c \in M_{y_{3}}$ be as given by Proposition 5.8 with $\lambda>2^{\kappa}>|T|$ : that is, for every $\gamma<\lambda$, we have $\left|S_{\psi, J_{>\gamma}}(I \times\{c\})\right|=2^{\kappa}$.

Let $V_{1,2}:=\left\{i \leq d:\left(t_{i}, s_{i}\right)=(1,2)\right\}, V_{1,3}:=\left\{i \leq d:\left(t_{i}, s_{i}\right)=(1,3)\right\}$ and $V_{2,3}:=\left\{i \leq d:\left(t_{i}, s_{i}\right)=\right.$ $(2,3)\}$; then $V_{1,2}, V_{1,3}, V_{2,3}$ is a partition of $\{1, \ldots, d\}$. Let $f\left(b_{\beta}, a_{\alpha}\right):=\left(f_{i}\left(b_{\beta}, a_{\alpha}\right): i \in V_{1,2}\right)$, $f\left(\bar{b}_{\beta}, c\right)=\left(f_{i}\left(c, b_{\beta}\right): i \in V_{1,3}\right)$ and $f\left(a_{\alpha}, c\right):=\left(f_{i}\left(a_{\alpha}, b_{\beta}\right): i \in V_{2,3}\right)$. Then we have

$$
\vDash \psi\left(b_{\beta} ; a_{\alpha}, c\right) \Longleftrightarrow \vDash \varphi^{\prime}\left(f\left(b_{\beta}, a_{\alpha}\right), f\left(b_{\beta}, c\right), f\left(a_{\alpha}, c\right)\right),
$$

where $\varphi^{\prime} \in \mathcal{L}_{0}$ is obtained from $\varphi$ by regrouping the variables accordingly.

Let $A:=\left\{f\left(a_{\alpha}, c\right): \alpha<\kappa\right\}$. Consider the rectangular array $\left(f\left(b_{\beta}, a_{\alpha}\right): \alpha<\kappa, \beta<\lambda\right)$. It is an indiscernible array by mutual indiscernibility of the sequences $\left(a_{\alpha}\right)$ and $\left(b_{\beta}\right)$. In particular, the sequence of rows $\left(\left(f\left(b_{\beta}, a_{\alpha}\right): \alpha<\kappa\right): \beta<\lambda\right)$ is $\emptyset$-indiscernible. As $T_{0}$ is dependent, $|A| \leq \kappa$ and $\lambda>(|T|+\kappa)$ is regular, there is some $\gamma<\lambda$ such that the sequence of columns $\left(\left(f\left(b_{\beta}, a_{\alpha}\right): \alpha<\kappa\right): \gamma<\beta<\lambda\right)$ is $\mathcal{L}_{0}$-indiscernible over $A$.

Fix $\gamma<\beta<\lambda$. For any tuple $e \in M_{\left(x_{i}: i \in V_{1,3}\right)}$, let

$$
S_{e}^{\beta}:=\left\{\alpha<\kappa: \vDash \varphi^{\prime}\left(f\left(b_{\beta}, a_{\alpha}\right), e, f\left(a_{\alpha}, c\right)\right)\right\} \subseteq \kappa,
$$

and let $\mathcal{F}_{\beta}:=\left\{S_{e}^{\beta}: e \in M_{\left(x_{i}: i \in V_{1,3}\right)}\right\}$ be the collection of all such subsets of $\kappa$ that can be realized by some tuple. 
We then have that $\mathcal{F}_{\beta}=\mathcal{F}_{\beta^{\prime}}$ for any $\gamma<\beta, \beta^{\prime}<\lambda$. Indeed, by the $\mathcal{L}_{0}$-indiscernibility observed above, there is some $\sigma \in \operatorname{Aut}\left(\mathbb{M}_{0} / A\right)$ sending $\left(f\left(b_{\beta}, a_{\alpha}\right): \alpha<\kappa\right)$ to $\left(f\left(b_{\beta}^{\prime}, a_{\alpha}\right): \alpha<\kappa\right)$. But then for any $e$, we have that $S_{e}^{\beta}=S_{\sigma(e)}^{\beta^{\prime}}$ (recalling that $f\left(a_{\alpha}, c\right) \in A$ for all $\left.\alpha<\kappa\right)$; hence $\mathcal{F}_{\beta} \subseteq \mathcal{F}_{\beta^{\prime}}$, and vice versa, exchanging the roles of $\beta$ and $\beta^{\prime}$. So let $\mathcal{F}:=\mathcal{F}_{\beta}$ for some (equivalently, any) $\beta>\gamma$. Note that $S_{e}^{\beta}$ is determined by the $\mathcal{L}_{0}$-type $\operatorname{tp}_{\varphi^{\prime}}\left(e /\left(f\left(b_{\beta}, a_{\alpha}\right): \alpha<\kappa\right) A\right)$. As $\left.\mid f\left(b_{\beta}, a_{\alpha}\right): \alpha<\kappa\right) A \mid \leq \kappa$ and $\varphi^{\prime}$ is dependent, we get that $|\mathscr{F}| \leq \operatorname{ded}(\kappa)$ by Fact 5.1.

Now we estimate $\left|S_{\psi, J_{>\gamma}}(I \times\{c\})\right|$ (see Definition 5.5). Given $\gamma<\beta<\lambda$, we have that $\operatorname{tp}_{\psi}\left(b_{\beta} / I \times\{c\}\right)$ is determined by the set

$$
S_{f\left(b_{\beta}, c\right)}^{\beta}=\left\{\alpha<\kappa: \vDash \varphi^{\prime}\left(f\left(b_{\beta}, a_{\alpha}\right), f\left(b_{\beta}, c\right), f\left(a_{\alpha}, c\right)\right)\right\} \in \mathcal{F} .
$$

But as $|\mathcal{F}| \leq \operatorname{ded}(\kappa)$, there are only $\operatorname{ded}(\kappa)$ choices for this set; hence $\left|S_{\psi, J_{>\gamma}}(I \times\{c\})\right| \leq \operatorname{ded}(\kappa)$.

This would imply a contradiction in a model of ZFC with $\operatorname{ded}(\kappa)<2^{\kappa}$ (which exists by Fact 5.2). But the property of a given formula $\psi$ being 2-dependent is arithmetic; hence set-theoretically absolute, so we obtain the result in ZFC.

Example 5.13. Let $f: \mathbb{C}^{2} \rightarrow \mathbb{C}$ be an arbitrary function, and let $p(x, y, z)$ be a polynomial over $\mathbb{C}$. Consider the relation $E \subseteq \mathbb{C}^{3}$ given by

$$
E(x, y, z) \Longleftrightarrow p(f(x, y), f(x, z), f(y, z))=0 .
$$

Then there is some finite 3-partite 3-hypergraph $H$ such that $E$ does not contain it as an induced tripartite hypergraph.

Remark 5.14. We will see in the proof of Theorem 6.3(3) that we cannot relax the assumption $\mathcal{M}_{0}$ is dependent to just 2-dependent. Generalizations of Theorem 5.12 for $n$-dependence and functions of arbitrary arity will be investigated in [12].

\section{2-dependence of bilinear forms over dependent fields}

In this section, we consider certain theories of bilinear forms on vector spaces, in a language with a separate sort for the field. Their basic model theory was studied by Granger in [21] and, more recently, in $[15,18]$ from the point of view of generalized stability theory. Here we investigate $n$-dependence in these structures. As it was already mentioned in the introduction, all currently known algebraic examples of strictly $n$-dependent theories for $n \geq 2$ are closely related to bilinear forms over finite fields. For example, smoothly approximable structures studied in [8] are 2-dependent and coordinatizable via bilinear forms over finite fields (see [14, Example 2.2(4)] for a discussion of their 2-dependence); and the strictly $n$ dependent pure groups constructed in [11] using Mekler's construction can be interpreted in alternating bilinear maps over finite fields as demonstrated in [3]. Here we show that a more general situation is possible: every non-degenerate (symmetric or alternating) bilinear form on an infinite dimensional vector space over an arbitrary dependent field is strictly 2-dependent (and the assumption that the field is dependent is necessary; see Theorem 6.3). Our proof of 2-dependence relies crucially on the Composition Lemma (Theorem 5.12) from the previous section. We view these examples as clarifying the scope of Conjecture 1.1 (namely, how much algebraic structure is required for the collapse of the $n$-dependence hierarchy) and guiding toward a correct formulation of its abstract counterpart ('every $n$-dependent theory is linear over its 1-dependent part').

We begin by recalling some definitions and results from [21]. In this section, we consider structures in the language $\mathcal{L}$ consisting of two sorts $V$ and $K$, the field language on $K$, the vector space language on $V$, scalar multiplication function $K \times V \rightarrow V$ and the bilinear form function $[x, y]: V \times V \rightarrow K$. The language $\mathcal{L}_{\theta}$ is obtained from $\mathcal{L}$ by adding for each $n \in \omega$ a (definable) $n$-ary predicate $\theta_{n}\left(x_{1}, \ldots, x_{n}\right)$, which holds if and only if $x_{1}, \ldots, x_{n} \in V$ are linearly independent over $K$. Let $\mathcal{L}_{\theta}^{K}$ be a language 
expanding $\mathcal{L}_{\theta}$ by relations on $K^{n}, n \in \omega$ definable in the language of rings such that $K$ eliminates quantifiers in $\mathcal{L}_{\theta}^{K}$ (e.g. we can always take Morleyzation of $K$ ).

Definition 6.1. For $K$ a field, $F \in\{A, S\}$ and an arbitrary $m \in \mathbb{N} \cup\{\infty\}$, let ${ }_{F} T_{m}^{K}$ denote the $\mathcal{L}_{\theta}^{K}$-theory expressing that the sort corresponding to $K$ is a field that is moreover a model of $\operatorname{Th}(K)$; $V$ a $K$-vector space of dimension $m ;[x, y]: V \times V \rightarrow K$ is a non-degenerate bilinear form of type $F$, where a form of type $S$ is a symmetric form, and a form of type $A$ is an alternating form; and the predicates $\theta_{n}$ define linear independent tuples of length $n$ over $K$.

Fact 6.2 [21, Theorem 9.2.3]. Let $F \in\{A, S\}$, and let $K$ be an arbitrary field if $F=A$, or a field closed under square roots if $F=S$. Let $m \in \mathbb{N} \cup\{\infty\}$ be arbitrary if $F=A$, or even if $m \in \mathbb{N}$ and $F=S$. Then the theory ${ }_{F} T_{m}^{K}$ is consistent, complete and has elimination of quantifiers in the language $\mathcal{L}_{\theta}^{K}$.

We are ready to state the main result of this section.

Theorem 6.3. Let $T:={ }_{F} T_{m}^{K}$ be as in Fact 6.2.

1. If $m<\infty$, then $\operatorname{Th}(K)$ is $n$-dependent if and only if $T$ is $n$-dependent, for any $n \in \mathbb{N}_{\geq 1}$.

2. If $m=\infty$ and $\operatorname{Th}(K)$ is dependent, then $T$ is strictly 2-dependent.

3. If $n \in \mathbb{N}_{\geq 1}, \operatorname{Th}(K)$ is not $n$-dependent and $m=\infty$, then $T$ is not $2 n$-dependent.

\section{Corollary 6.4.}

1. The case of a finite field $K$ corresponding to extra-special p-groups was treated in [22, Section 3].

2. In [4], for each $n \in \mathbb{N}$ and $p$, Baudisch constructs a structure $D(n)$ in the language of groups with $n$ additional constant symbols, with $D(1)$ corresponding to extra-special p-groups. Since all these examples are interpretable in the bilinear form with additional constant symbols, they are all 2-dependent.

The rest of the section constitutes a proof of this theorem.

\subsection{Proof of Theorem 6.3(1)}

If $m<\infty$, then $K$ is $n$-dependent if and only if $T$ is $n$-dependent, for any $n \geq 1$. This follows from the fact that any model of ${ }_{F} T_{m}^{K}$ can be interpreted in $K$ using the isomorphism $K^{m} \cong V$ for some $m \in \mathbb{N}$ as follows. Interpreting the vector space structure is obvious. Now, let $\mathcal{B}=\left(e_{1}, \ldots, e_{m}\right)$ be the standard basis of $K^{m}$. Then the bilinear form is completely determined by fixing $k_{i, j}=\left[e_{i}, e_{j}\right]$ for all $1 \leq i, j \leq m$. Let $\pi_{i}: K^{m} \rightarrow K$ be the projection map onto the $i$-th coordinate. Then for $v, w \in K^{m}$, we have that $[v, w]=\sum_{i, j=1}^{m} \pi_{i}(v) \pi_{j}(w) k_{i, j}$, which is definable over $\left\{k_{i, j}: 1 \leq i, j \leq m\right\}$.

\subsection{Proof of Theorem 6.3(3)}

Assume that $K$ is not $n$-dependent; then by Theorem 2.12, it must be witnessed by some $\mathcal{L}_{K}$-formula $\varphi\left(\bar{x} ; y_{1}, \ldots, y_{n}\right)$ with each $y_{i}$ a single variable. Then by compactness for $1 \leq k \leq n$, we can find sequences $\left(c_{\left(i_{k}, j_{k}\right)}^{k}:\left(i_{k}, j_{k}\right) \in \omega \times \omega\right)$ with $\omega \times \omega$ ordered lexicographically and all $c_{i_{k}, j_{k}}^{k}$ pairwise distinct elements in $K$, such that for every $A \subseteq(\omega \times \omega)^{n}$, there is some $\bar{e}_{A}$ satisfying

$$
\vDash \varphi\left(\bar{e}_{A} ; c_{\left(i_{1}, j_{1}\right)}^{1}, \ldots, c_{\left(i_{n}, j_{n}\right)}^{n}\right) \Longleftrightarrow\left(\left(i_{1}, j_{1}\right), \ldots,\left(i_{n}, j_{n}\right)\right) \in A .
$$

As $m=\infty$, we can choose $\left(a_{i}^{k}: 1 \leq k \leq n, i \in \omega\right)$ a tuple consisting of linearly independent elements in $V$. For each $1 \leq k \leq n$ and $j \in \omega$, let $f_{j}^{k}: V \rightarrow K$ be a linear function satisfying $f_{j}^{k}\left(a_{i}^{k}\right)=c_{(i, j)}^{k}$ for all $i \in \omega$. Since the bilinear form is non-degenerate, there exist some $b_{j}^{k} \in V$ such that $f_{j}^{k}(x)=\left[x, b_{j}^{k}\right]$ for all $x \in V$. But then, identifying $(\omega \times \omega)^{n}$ with $\omega^{2 n}$, for any set $A \subseteq \omega^{2 n}$, we have

$$
\vDash \varphi\left(\bar{e}_{A},\left[a_{i_{1}}^{1}, b_{j_{1}}^{1}\right], \ldots,\left[a_{i_{n}}^{n}, b_{j_{n}}^{n}\right]\right) \Longleftrightarrow\left(i_{1}, j_{1}, \ldots, i_{n}, j_{n}\right) \in A,
$$


hence the formula $\psi\left(\bar{x} ; y_{1}, y_{2}, \ldots, y_{2 n-1}, y_{2 n}\right)=\varphi\left(\bar{x},\left[y_{1}, y_{2}\right], \ldots,\left[y_{2 n-1}, y_{2 n}\right]\right)$ is not $2 n$-dependent, witnessed by the sequences

$$
\left(a_{i_{1}}^{1}, b_{j_{1}}^{1}, \ldots, a_{i_{n}}^{n}, b_{j_{n}}^{n}: i_{1}, j_{1}, \ldots, i_{n}, j_{n} \in \omega\right) .
$$

\subsection{Proof of Theorem 6.3(2)}

Let $\mathbb{M} \models T$ be a monster model. If $T$ is not 2-dependent, by Proposition 2.8 there exists tuples $\bar{a}_{\alpha}, \bar{b}_{\beta}$ and an $\mathcal{L}_{\theta}^{K}$-formula $\varphi(\bar{x} ; \bar{y}, \bar{z})$ without parameters such that $\left(\bar{a}_{\alpha}, \bar{b}_{\beta}: \alpha, \beta \in \mathbb{Q}\right)$ is $O_{2, p}$-indiscernible over $\emptyset$ and it is shattered by $\varphi$. More precisely, for every $A \subseteq \mathbb{Q} \times \mathbb{Q}$, there is some $\bar{c}_{A}$ such that

$$
\vDash \varphi\left(\bar{c}_{A} ; \bar{a}_{\alpha}, \bar{b}_{\beta}\right) \Longleftrightarrow(\alpha, \beta) \in A .
$$

We write $\bar{x}=\bar{x}^{K} \frown \bar{x}^{V}, \bar{y}=\bar{y}^{K} \frown \bar{y}^{V}, \bar{z}=\bar{z}^{K} \frown \bar{z}^{V}$ for the subtuples of the variables of the corresponding sorts, where $\bar{x}^{K}=\left(x_{i}^{K}: i \in X^{K}\right)$ and $\bar{x}^{V}=\left(x_{i}^{V}: i \in X^{V}\right)$ and $X^{K} \sqcup X^{V}$ is a partition of $\{1, \ldots,|\bar{x}|\}$. We proceed similarly for $\bar{y}$ and $\bar{z}$. Let $a_{\alpha}=\bar{a}_{\alpha}^{K} \bar{a}_{\alpha}^{V}, \bar{b}_{\beta}=\bar{b}_{\beta}^{K \frown} \bar{b}_{\beta}^{V}$ for the corresponding subtuples in the $K$-sort and the $V$-sort, respectively. Let $\bar{a}_{\alpha}^{V}=\left(a_{\alpha, i}^{V}: i \in Y^{V}\right), \bar{b}_{\beta}^{V}=\left(b_{\beta, i}^{V}: i \in Z^{V}\right)$, etc.

As a first step toward obtaining a contradiction, we will show that all of the elements of the sort $V$ in the configuration witnessing the failure of 2-dependence chosen above may be assumed linearly independent over $K$. This is achieved by modifying the initial configuration and the formula as demonstrated in the following four claims.

Claim 6.5. There exist a finite tuple $\bar{e}$ in $V$, a formula $\varphi(\bar{x}, \bar{w}, \bar{y}, \bar{z}) \in \mathcal{L}_{\theta}^{K}$ and sequences of tuples $\left(\bar{a}_{\alpha}, \bar{b}_{\beta}: \alpha, \beta \in \mathbb{Q}\right)$ such that:

1. $\left(\bar{a}_{\alpha}, \bar{b}_{\beta}: \alpha, \beta \in \mathbb{Q}\right)$ is $O_{2, p}$-indiscernible over $\bar{e}$;

2. For any $\alpha^{*} \in \mathbb{Q}$ and $i^{*} \in Y^{V}$, we have that

$$
a_{\alpha^{*}, i^{*}}^{V} \notin \operatorname{Span}\left(\left(a_{\alpha^{*}, i}^{V}: i \in Y^{V} \backslash\left\{i^{*}\right\}\right),\left(\bar{a}_{\alpha}^{V}: \alpha \in \mathbb{Q} \backslash\left\{\alpha^{*}\right\}\right),\left(\bar{b}_{\beta}^{V}: \beta \in \mathbb{Q}\right), \bar{e}\right) ;
$$

3. For any $\beta^{*} \in \mathbb{Q}$ and $j^{*} \in Z^{V}$, we have that

$$
b_{\beta^{*}, j^{*}}^{V} \notin \operatorname{Span}\left(\left(b_{\beta^{*}, j}^{V}: j \in Z^{V} \backslash\left\{j^{*}\right\}\right),\left(\bar{b}_{\beta}^{V}: \beta \in \mathbb{Q} \backslash\left\{\beta^{*}\right\}\right),\left(\bar{a}_{\alpha}^{V}: \alpha \in \mathbb{Q}\right), \bar{e}\right) ;
$$

4. $\varphi(\bar{x}, \bar{e} ; \bar{y}, \bar{z})$ shatters $\left(\bar{a}_{\alpha}, \bar{b}_{\beta}: \alpha, \beta \in \mathbb{Q}\right)$ - that is, for every $A \subseteq \mathbb{Q}^{2}$, there is some $\bar{c}_{A}$ such that $\vDash \varphi\left(c_{A}, \bar{e}, \bar{a}_{\alpha}, \bar{b}_{\beta}\right) \Longleftrightarrow(\alpha, \beta) \in A$.

Proof. Assume that $\left(\bar{a}_{\alpha}, \bar{b}_{\beta}\right)$ chosen in the beginning of the proof do not satisfy (2) with $\bar{e}=\emptyset$. Then there are some $\alpha^{*} \in \mathbb{Q}, i^{*} \in Y^{V}$ and finite sets $I, J \subseteq \mathbb{Q}, \alpha^{*} \notin I$ such that

$$
a_{\alpha^{*}, i^{*}}^{V} \in \operatorname{Span}\left(\left(a_{\alpha^{*}, i}^{V}: i \in Y^{V} \backslash\left\{i^{*}\right\}\right),\left(\bar{a}_{\alpha}^{V}\right)_{\alpha \in I},\left(\bar{b}_{\beta}^{V}\right)_{\beta \in J}, \bar{e}\right) .
$$

Then there is an $\emptyset$-definable function $f$ and some finite tuple $\bar{k}$ in $K$ such that

$$
a_{\alpha^{*}, i^{*}}^{V}=f\left(\bar{k},\left(a_{\alpha^{*}, i}^{V}: i \in Y^{V} \backslash\left\{i^{*}\right\}\right),\left(\bar{a}_{\alpha}^{V}\right)_{\alpha \in I},\left(\bar{b}_{\beta}^{V}\right)_{\beta \in J}, \bar{e}\right) .
$$

We let

$$
\begin{gathered}
\gamma^{+}:=\min \left\{\alpha \in I: \alpha>\alpha^{*}\right\}, \gamma^{-}:=\max \left\{\alpha \in I: \alpha<\alpha^{*}\right\}, \delta:=\max (J), \\
\bar{e}^{\prime}:=\left(\bar{a}_{\alpha}^{V}\right)_{\alpha \in I} \frown\left(\bar{b}_{\beta}^{V}\right)_{\beta \in J} \frown \bar{e}, \\
\left(\bar{a}^{\prime}\right)_{\alpha}^{V}:=\left(a_{\alpha^{*}, i}^{V}: i \in Y^{V} \backslash\left\{i^{*}\right\}\right) .
\end{gathered}
$$


Then

$$
\left(\bar{a}_{\alpha}^{K \frown}\left(\bar{a}^{\prime}\right)_{\alpha}^{V}, \bar{b}_{\beta}: \alpha \in\left(\gamma^{-}, \gamma^{+}\right), \beta \in(\delta,+\infty)\right)
$$

is $O_{2, p^{-}}$-indiscernible over $\bar{e}^{\prime}$. As $\alpha^{*} \in\left(\gamma^{-}, \gamma^{+}\right)$, it follows that for every $\alpha \in\left(\gamma^{-}, \gamma^{+}\right)$, there is some tuple $\bar{k}_{\alpha}$ in $K$ such that $a_{\alpha, i^{*}}^{V}=f\left(\bar{k}_{\alpha},\left(\bar{a}^{\prime}\right)_{\alpha}^{V}, \bar{e}^{\prime}\right)$. Let

$$
\varphi^{\prime}\left(\bar{x}, \bar{w}, \bar{y}^{\prime}, \bar{z}\right):=\varphi\left(\bar{x} ; \bar{y}^{K},\left(y_{i}^{V}\right)_{i \in Y^{V}, i<i^{*}}, f\left(\bar{y}_{1}^{K},\left(y_{i}^{V}\right)_{i \in Y^{V} \backslash\left\{i^{*}\right\}}, \bar{w}\right),\left(y_{i}^{V}\right)_{i \in Y^{V}, i>i^{*}}, \bar{z}\right),
$$

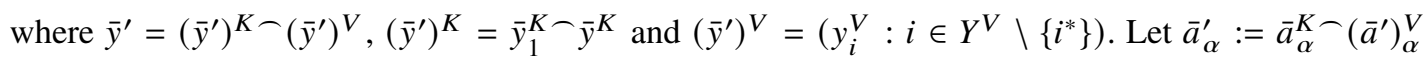
(so the tuple $\mathrm{k}_{\alpha} \bar{a}_{\alpha}^{\prime}$ corresponds to the variables $\bar{y}^{\prime}$ ).

Restricting to the set $\left(\gamma^{-}, \gamma^{+}\right) \times(\delta, \infty)$, we may thus assume:

(a) $\left(\bar{a}_{\alpha}^{\prime}, \bar{b}_{\beta}: \alpha, \beta \in \mathbb{Q}\right)$ is $O_{2, p}$-indiscernible over $\bar{e}^{\prime}$ (follows by the choice of $\gamma^{-}, \gamma^{+}, \delta$, definition of $\bar{a}_{\alpha}^{\prime}$ and $O_{2, p}$-indiscernibility of $\left.\left(\bar{a}_{\alpha}, \bar{b}_{\beta}: \alpha, \beta \in \mathbb{Q}\right)\right)$,

(b) $\varphi^{\prime}\left(\bar{x}, \bar{e}^{\prime} ; \bar{y}^{\prime}, \bar{z}\right)$ shatters $\left(\bar{k}_{\alpha} \bar{a}_{\alpha}^{\prime}, \bar{b}_{\beta}: \alpha, \beta \in \mathbb{Q}\right.$ ) (as for any $\bar{c}$ and $\alpha, \beta \in \mathbb{Q}$, by the above we have $\left.\vDash \varphi\left(\bar{c}, \bar{a}_{\alpha}, \bar{b}_{\beta}\right) \Longleftrightarrow \vDash \varphi^{\prime}\left(\bar{c}, \bar{e}^{\prime}, \bar{k}_{\alpha} \bar{a}_{\alpha}^{\prime}, \bar{b}_{\beta}\right)\right)$.

By Fact 2.7 , let $\left(\bar{h}_{\alpha} \bar{a}_{\alpha}^{\prime \prime}, \bar{b}_{\beta}^{\prime}: \alpha, \beta \in \mathbb{Q}\right)$ be an $O_{2, p}$-indiscernible over $\bar{e}^{\prime}$, based on $\left(\bar{k}_{\alpha} \bar{a}_{\alpha}^{\prime}, \bar{b}_{\beta}\right.$ : $\alpha, \beta \in \mathbb{Q})$ over $\bar{e}^{\prime}$. We still have that $\varphi^{\prime}\left(\bar{x}, \bar{e}^{\prime}, \bar{y}^{\prime}, \bar{z}\right)$ shatters $\left(\bar{h}_{\alpha} \bar{a}_{\alpha}^{\prime \prime}, \bar{b}_{\beta}^{\prime}: \alpha, \beta \in \mathbb{Q}\right)$. Replacing $\varphi$ by $\varphi^{\prime}$, $\bar{e}$ by $\bar{e}^{\prime}$ and the sequences $\left(\bar{a}_{\alpha}, \bar{b}_{\beta}: \alpha, \beta \in \mathbb{Q}\right)$ by $\left(\bar{h}_{\alpha} \bar{a}_{\alpha}^{\prime \prime}, \bar{b}_{\beta}^{\prime}: \alpha, \beta \in \mathbb{Q}\right)$, we have thus reduced the length of the tuples $\bar{a}_{\alpha}^{V}$ (at the price of increasing the length of $\bar{a}_{\alpha}^{K}$ ). Repeating this argument for both $\bar{a}_{\alpha}$ 's and $\bar{b}_{\beta}$ 's at most finitely many times (as the variables $\bar{y}$ and $\bar{z}$ have finite length), we obtain the conclusion of the claim.

Claim 6.6. There exist finite tuples $\bar{e}$ in $V$ and $\bar{c}$, a formula $\varphi(\bar{x}, \bar{w}, \bar{y}, \bar{z}) \in \mathcal{L}_{\theta}^{K}$ and sequences of tuples $\left(\bar{a}_{\alpha}, \bar{b}_{\beta}: \alpha, \beta \in \mathbb{Q}\right)$ satisfying (1)-(3) and

$\left(4^{\prime}\right) \vDash \varphi\left(\bar{c}, \bar{e}, \bar{a}_{\alpha}, \bar{b}_{\beta}\right) \Longleftrightarrow G_{2, p} \vDash R_{2}(\alpha, \beta)$ for all $\alpha, \beta \in \mathbb{Q}$.

5. $c_{i}^{V} \notin \operatorname{Span}\left(\left(c_{j}^{V}: j \neq i\right),\left(\bar{a}_{\alpha}^{V}\right)_{\alpha \in \mathbb{Q}},\left(\bar{b}_{\beta}^{V}\right)_{\beta \in \mathbb{Q}}, \bar{e}\right)$ for any $i \in X^{V}$.

Proof. We start with $\bar{e}, \varphi$ and $\left(\bar{a}_{\alpha}, \bar{b}_{\beta}\right)_{\alpha, \beta \in \mathbb{Q}}$ satisfying (1)-(4) of Claim 6.5. By (4), there exists a tuple $\bar{c}$ such that

$$
\vDash \varphi\left(\bar{c}, \bar{e}, \bar{a}_{\alpha}, \bar{b}_{\beta}\right) \Longleftrightarrow G_{2, p} \vDash R_{2}(\alpha, \beta) \text { for all } \alpha, \beta \in \mathbb{Q} \text {. }
$$

We write $\bar{c}=\bar{c}^{K \frown \bar{c}^{V}}$. Assume that there is some $i^{*} \in X^{V}$ such that $c_{i^{*}}^{V}$ is in the span of the tuple

$$
\left(c_{i}^{V}: i \in X^{V} \backslash\left\{i^{*}\right\}\right)^{\frown}\left(\bar{a}_{\alpha}^{V}\right)_{\alpha \in I} \frown\left(\bar{b}_{\beta}^{V}\right)_{\beta \in J} \frown \bar{e}
$$

for some finite sets $I, J \subseteq \mathbb{Q}$. Then

$$
c_{i^{*}}^{V}=f\left(\bar{c}_{1}^{K},\left(c_{i}^{V}\right)_{i \in X^{V} \backslash\left\{i^{*}\right\}},\left(\bar{a}_{\alpha}^{V}\right)_{\alpha \in I},\left(\bar{b}_{\beta}^{V}\right)_{\beta \in J}, \bar{e}\right)
$$

for some $\emptyset$-definable function $f$ and some tuple $\bar{c}_{1}^{K}$ in $K$. Let $\alpha^{*}:=\max (I \cup J)$. We let $\bar{c}^{\prime}:=\left(\bar{c}^{\prime}\right)^{K}-\left(\bar{c}^{\prime}\right)^{V}$, where $\left(\bar{c}^{\prime}\right)^{K}:=\bar{c}_{1}^{K} \frown \bar{c}^{K}$ and $\left(\bar{c}^{\prime}\right)^{V}:=\left(c_{i}^{V}: i \in X^{V} \backslash\left\{i^{*}\right\}\right)$. Let $\bar{e}^{\prime}:=\left(\bar{a}_{\alpha}^{V}\right)_{\alpha \in I} \frown\left(\bar{b}_{\beta}^{V}\right)_{\beta \in J} \frown \bar{e}$. Restricting to a copy of $G_{2, p}$ contained in $\left(\alpha^{*}, \infty\right) \times\left(\alpha^{*}, \infty\right)$ (Remark 2.5), we thus have:

$$
\begin{gathered}
G_{2, p} \vDash R_{2}(\alpha, \beta) \Longleftrightarrow \vDash \varphi\left(\bar{c}, \bar{e}, \bar{a}_{\alpha}, \bar{b}_{\beta}\right) \Longleftrightarrow \\
\vDash \varphi\left(\bar{c}^{K},\left(\bar{c}_{i}^{V}\right)_{i<i^{*}}, f\left(\bar{c}_{1}^{K},\left(\bar{c}^{\prime}\right)^{V}, \bar{e}^{\prime}\right),\left(\bar{c}_{i}^{V}\right)_{i>i^{*}}, \bar{e}, \bar{a}_{\alpha}, \bar{b}_{\beta}\right) \\
\Longleftrightarrow \vDash \varphi^{\prime}\left(\bar{c}^{\prime}, \bar{e}^{\prime}, \bar{a}_{\alpha}, \bar{b}_{\beta}\right)
\end{gathered}
$$


for an appropriate $\mathcal{L}_{\theta}^{K}$-formula $\varphi^{\prime}$. Replacing $\varphi$ by $\varphi^{\prime}, \bar{e}$ by $\bar{e}^{\prime}$ and $\bar{c}$ by $\bar{c}^{\prime}$, this shows that (4') is still satisfied. And (1), (2) and (3) still hold as well (follows as $\bar{e}$ satisfies (1), (2), (3) and all the new elements in $\bar{e}^{\prime}$ are from $\left.\left(\bar{a}_{\alpha}^{\prime}, \bar{b}_{\beta}^{\prime}: \alpha, \beta<\alpha^{*}\right)\right)$. We have thus reduced the length of the tuple $\bar{c}$ (at the price of increasing the length of $\bar{e}$ ). Repeating this argument finitely many times if necessary, we obtain the claim.

Claim 6.7. There exist finite tuples $\bar{e}$ in $V$ and $\bar{c}$, a formula $\varphi(\bar{x}, \bar{w}, \bar{y}, \bar{z}) \in \mathcal{L}_{\theta}^{K}$ and sequences of tuples $\left(\bar{a}_{\alpha}, \bar{b}_{\beta}: \alpha, \beta \in \mathbb{Q}\right)$ satisfying (1)-(3), (4'), (5) and

6. $e_{i} \notin \operatorname{Span}\left(e_{j}: j \neq i\right)$ for any $i \in|\bar{e}|$.

Proof. Start with $\bar{e}, \varphi$ and $\left(\bar{a}_{\alpha}, \bar{b}_{\beta}\right)_{\alpha, \beta \in \mathbb{Q}}$ given by Claim 6.6. As in the previous two claims, if $e_{i^{*}} \in \operatorname{Span}\left(\bar{e}_{i}: i \neq i^{*}\right)$, then $e_{i^{*}}=f\left(\bar{k},\left(\bar{e}_{i}\right)_{i \neq i^{*}}\right)$ for some $\emptyset$-definable function $f$ and a tuple $\bar{k}$ in $K$. Replacing $\bar{e}$ by $\left(\bar{e}_{i}\right)_{i \neq i^{*}}$, adding $\bar{k}$ to $\bar{c}^{K}$ and modifying the formula accordingly, the condition (4') is still satisfied. And (1), (2), (3), (5), (6) still hold as we only pass to a subtuple of $\bar{e}$. Repeating this finitely many times, we obtain the claim.

Let $\bar{e}, \bar{c}, \varphi(\bar{x}, \bar{w}, \bar{y}, \bar{z}) \in \mathcal{L}_{\theta}^{K}$ and $\left(\bar{a}_{\alpha}, \bar{b}_{\beta}: \alpha, \beta \in \mathbb{Q}\right)$ be as given by Claim 6.7. By (2), (3), (5), (6) and linear algebra we get that all elements in the tuple

$$
\bar{c}^{V} \frown\left(\bar{a}_{\alpha}^{V}\right)_{\alpha \in \mathbb{Q}} \frown\left(\bar{b}_{\beta}^{V}\right)_{\beta \in \mathbb{Q}} \frown \bar{e}
$$

are linearly independent. Hence adjoining $\bar{e}^{V}$ to $\bar{c}^{V}$ and regrouping the variables of $\varphi$ accordingly, we get:

Claim 6.8. There is an $\mathcal{L}_{\theta}^{K}$-formula $\varphi(\bar{x}, \bar{y}, \bar{z})$ and tuples $\bar{c}, \bar{a}_{\alpha}, \bar{b}_{\beta}$ such that:

1. $\left(\bar{a}_{\alpha}, \bar{b}_{\beta}: \alpha, \beta \in \mathbb{Q}\right)$ is $O_{2, p}$-indiscernible;

2. All elements in the tuple $\bar{c}^{V} \frown\left(\bar{a}_{\alpha}^{V}\right)_{\alpha \in \mathbb{Q}} \frown\left(\bar{b}_{\beta}^{V}\right)_{\beta \in \mathbb{Q}}$ are linearly independent;

3. $\vDash \varphi\left(\bar{c}, \bar{a}_{\alpha}, \bar{b}_{\beta}\right) \Longleftrightarrow G_{2, p} \vDash R_{2}(\alpha, \beta)$.

By quantifier elimination (Fact 6.2), every $\mathcal{L}_{\theta}^{K}$-formula is equivalent to a Boolean combination of atomic $\mathcal{L}_{\theta}^{K}$-formulas. Since 2-dependence is preserved under Boolean combinations (Fact 2.2), it is sufficient to check 2-dependence for atomic $\mathcal{L}_{\theta}^{K}$-formulas, which by the definition of the language $\mathcal{L}_{\theta}^{K}$ fall into one of the following three cases.

Case 1. The formula $\varphi(\bar{x}, \bar{y}, \bar{z})$ is of the form $\psi\left(t_{1}(\bar{x}, \bar{y}, \bar{z}), \ldots, t_{d}(\bar{x}, \bar{y}, \bar{z})\right)$ for some $\psi \in \mathcal{L}_{K}$ and some terms $t_{l}(\bar{x}, \bar{y}, \bar{z})$ taking values in $K$ and $1 \leq l \leq d$.

In this case, for each $l$, we have the following possibilities:

- The term $t_{l}(\bar{x}, \bar{y}, \bar{z})$ has height 1 - that is, it is one of the variables in $\bar{x}^{K} \frown \bar{y}^{K}-\bar{z}^{K}$;

$\circ t_{l}(\bar{x}, \bar{y}, \bar{z})=t_{l}^{1}(\bar{x}, \bar{y}, \bar{z})+_{K} t_{l}^{2}(x, \bar{y}, \bar{z})$ or $t_{l}(\bar{x}, \bar{y}, \bar{z})=t_{l}^{1}(\bar{x}, \bar{y}, \bar{z}) \cdot_{K} t_{l}^{2}(\bar{x}, \bar{y}, \bar{z})$, for some terms $t_{l}^{1}, t_{l}^{2}$ of smaller height taking values in $K$;

$\circ t_{l}(\bar{x}, \bar{y}, \bar{z})=\left[t_{l}^{1}(\bar{x}, \bar{y}, \bar{z}), t_{l}^{2}(\bar{x}, \bar{y}, \bar{z})\right]$ for some terms $t_{l}^{1}, t_{l}^{2}$ of smaller height taking values in $V$, but then:

- Either $t_{l}^{1}$ is of height 1 - that is, it is one of the variables in $\bar{x}^{V \frown} \bar{y}^{V \frown} \bar{z}^{V}$;

- Or $t_{l}^{1}(\bar{x}, \bar{y}, \bar{z})=s_{l}^{1}(\bar{x}, \bar{y}, \bar{z}) \cdot V s_{l}^{2}(\bar{x}, \bar{y}, \bar{z})$ for some terms $s_{l}^{1}, s_{l}^{2}$ of smaller height taking values in $K$ and $V$, respectively, in which case $t_{l}(\bar{x}, \bar{y}, \bar{z})=s_{l}^{1}(\bar{x}, \bar{y}, \bar{z}) \cdot_{K}\left[s_{l}^{2}(\bar{x}, \bar{y}, \bar{z}), t_{l}^{2}(\bar{x}, \bar{y}, \bar{z})\right]$;

- Or $t_{l}^{1}(\bar{x}, \bar{y}, \bar{z})=s_{l}^{1}(\bar{x}, \bar{y}, \bar{z})+_{V} s_{l}^{2}(\bar{x}, \bar{y}, \bar{z})$ for some terms $s_{l}^{1}, s_{l}^{2}$ of smaller height taking values in $V$, in which case

$$
t_{l}(\bar{x}, \bar{y}, \bar{z})=\left[s_{l}^{1}(\bar{x}, \bar{y}, \bar{z}), t_{l}^{2}(\bar{x}, \bar{y}, \bar{z})\right]+_{K}\left[s_{l}^{2}(\bar{x}, \bar{y}, \bar{z}), t_{l}^{2}(\bar{x}, \bar{y}, \bar{z})\right] .
$$

And similarly for $t_{l}^{2}$. 
Applying this for each $l$ and iterating by recursion on the height of terms, we thus conclude that the formula $\psi\left(t_{1}(\bar{x}, \bar{y}, \bar{z}), \ldots, t_{d}(\bar{x}, \bar{y}, \bar{z})\right)$ is equivalent to

$$
\begin{aligned}
& \psi^{\prime}\left(\left(\left[x_{i}^{V}, y_{j}^{V}\right]\right)_{i \in X^{V}, j \in Y^{V}},\left(\left[x_{i}^{V}, z_{j}^{V}\right]\right)_{i \in X^{V}, j \in Y^{V}},\left(\left[y_{i}^{V}, z_{j}^{V}\right]\right)_{i \in Y^{V}, j \in Z^{V}},\right.
\end{aligned}
$$

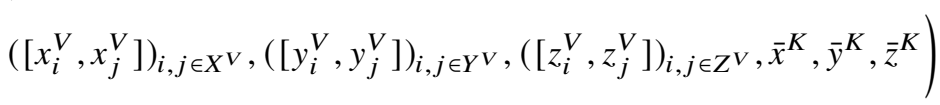

for some $\mathcal{L}_{K}$-formula $\psi^{\prime}$. As $\operatorname{Th}(K)$ is dependent, Theorem 5.12 implies that this formula is 2-dependent. This concludes Case 1.

Case 2. The formula $\varphi(\bar{x} ; \bar{y}, \bar{z})$ is given by

$$
\theta_{d}\left(t_{1}(\bar{x}, \bar{y}, \bar{z}), \ldots, t_{d}(\bar{x}, \bar{y}, \bar{z})\right)
$$

for some $d \in \mathbb{N}$ and terms $t_{l}(\bar{x}, \bar{y}, \bar{z})$ taking values in $V$.

In this case, by a simple recursion on the height of the terms, we see that for $1 \leq l \leq d$, the term $t_{l}$ must be of the form

$$
\sum_{i \in X^{V}} t_{l, i}^{X}(\bar{x}, \bar{y}, \bar{z}) x_{i}^{V}+\sum_{j \in Y^{V}} t_{l, j}^{Y}(\bar{x}, \bar{y}, \bar{z}) y_{j}^{V}+\sum_{k \in Z^{V}} t_{l, k}^{Z}(\bar{x}, \bar{y}, \bar{z}) z_{k}^{V}
$$

for some terms $t_{l, i}^{X}, t_{l, j}^{Y}, t_{l, k}^{Z}$ taking values in $K$.

Using linear independence of the set of elements of the tuple $\bar{c} V \frown \bar{a}_{\alpha}^{V}-\bar{b}_{\beta}^{V}$ for any $\alpha, \beta \in \mathbb{Q}$ established in Claim 6.8, for any $\alpha, \beta \in \mathbb{Q}$, we have

$$
\begin{gathered}
G_{2, p} \vDash R_{2}(\alpha, \beta) \Longleftrightarrow \vDash \neg \theta_{d}\left(t_{1}\left(\bar{c}, \bar{a}_{\alpha}, \bar{b}_{\beta}\right), \ldots, t_{d}\left(\bar{c}, \bar{a}_{\alpha}, \bar{b}_{\beta}\right)\right) \Longleftrightarrow \\
\vDash\left(\exists e_{1}, \ldots, e_{d} \in K\right)\left(e_{1}, \ldots, e_{d}\right) \neq(0, \ldots, 0) \wedge \bigwedge_{i \in X^{V}}\left(\sum_{l=1}^{d} e_{l} \cdot t_{l, i}^{X}\left(\bar{c}, \bar{a}_{\alpha}, \bar{b}_{\beta}\right)=0\right) \wedge \\
\bigwedge_{j \in Y^{V}}\left(\sum_{l=1}^{d} e_{l} \cdot t_{l, j}^{Y}\left(\bar{c}, \bar{a}_{\alpha}, \bar{b}_{\beta}\right)=0\right) \wedge \bigwedge_{k \in Z^{V}}\left(\sum_{l=1}^{d} e_{l} \cdot t_{l, k}^{Z}\left(\bar{c}, \bar{a}_{\alpha}, \bar{b}_{\beta}\right)=0\right) \Longleftrightarrow \\
\vDash \psi\left(\left(t_{l, i}^{X}\left(\bar{c}, \bar{a}_{\alpha}, \bar{b}_{\beta}\right)\right)_{1 \leq l \leq d, i \in X^{V}},\left(t_{l, j}^{Y}\left(\bar{c}, \bar{a}_{\alpha}, \bar{b}_{\beta}\right)\right)_{1 \leq l \leq d, j \in Y^{V}},\left(t_{l, k}^{Z}\left(\bar{c}, \bar{a}_{\alpha}, \bar{b}_{\beta}\right)\right)_{1 \leq l \leq d, k \in Z^{V}}\right)
\end{gathered}
$$

for an appropriate formula $\psi \in \mathcal{L}_{K}$. But this is impossible by Case 1 .

Case 3. The formula is of the form $t(\bar{x}, \bar{y}, \bar{z})=0$ for some term.

As in the previous case, then $t$ must be of the form

$$
\sum_{i \in X^{V}} t_{i}^{X}(\bar{x}, \bar{y}, \bar{z}) x_{i}^{V}+\sum_{j \in Y^{V}} t_{j}^{Y}(\bar{x}, \bar{y}, \bar{z}) y_{j}^{V}+\sum_{k \in Z^{V}} t_{k}^{Z}(\bar{x}, \bar{y}, \bar{z}) z_{k}^{V}
$$

for some terms $t_{i}^{X}, t_{j}^{Y}, t_{k}^{Z}$ taking values in $K$. Using again linear independence of $\bar{c}^{V} \frown \bar{a}_{\alpha}^{V} \frown \bar{b}_{\beta}^{V}$ for any $\alpha, \beta \in \mathbb{Q}$ established in Claim 6.8, we have that

$$
\begin{gathered}
G_{2, p} \vDash R_{2}(\alpha, \beta) \Longleftrightarrow t\left(\bar{c}, \bar{a}_{\alpha}, \bar{b}_{\beta}\right)=0 \Longleftrightarrow \\
\bigwedge_{i \in X^{V}} t_{i}^{X}\left(\bar{c}, \bar{a}_{\alpha}, \bar{b}_{\beta}\right)=0 \wedge \bigwedge_{j \in Y^{V}} t_{j}^{Y}\left(\bar{c}, \bar{a}_{\alpha}, \bar{b}_{\beta}\right)=0 \wedge \bigwedge_{k \in Z^{V}} t_{k}^{Z}\left(\bar{c}, \bar{a}_{\alpha}, \bar{b}_{\beta}\right)=0 \\
\Longleftrightarrow \psi\left(\left(t_{i}^{X}\left(\bar{c}, \bar{a}_{\alpha}, \bar{b}_{\beta}\right)\right)_{i \in X^{V}},\left(t_{j}^{Y}\left(\bar{c}, \bar{a}_{\alpha}, \bar{b}_{\beta}\right)\right)_{j \in Y^{V}},\left(t_{k}^{Z}\left(\bar{c}, \bar{a}_{\alpha}, \bar{b}_{\beta}\right)\right)_{k \in Z^{V}}\right)
\end{gathered}
$$


for an appropriate $\mathcal{L}_{K}$-formula $\psi$ and any $\alpha, \beta \in \mathbb{Q}$ - which is impossible by Case 1 .

This finishes the proof of Theorem 6.3(2).

\section{Expansions by generic predicates and $n$-dependence}

In this section, we will show that an expansion of a geometric theory $T$ by a generic predicate, in the sense of [6], is dependent if and only if it is $n$-dependent for some $n$, if and only if the algebraic closure in $T$ is disintegrated (Corollary 7.13). In fact, we prove that any expansion of an $n$-dependent geometric theory with disintegrated algebraic closure by generic relations of arity at most $n$ is $n$-dependent (Proposition 7.12). While these results have no direct implication for Conjecture 1.1, the authors view it as providing some further evidence toward it. Namely, we view it as a 'toy example' of a situation in which failure of 1-dependence implies failure of $n$-dependence for all $n$, utilizing the geometric complexity (nondisintegration) of the algebraic closure, similar to the behavior expected in fields.

We begin by recalling some basics of geometric theories and expansions by generic predicates from [6]. A theory $T$ is geometric if it eliminates the $\exists^{\infty}$-quantifier and the algebraic closure operator acl satisfies exchange in every model of $T$. In this section, we denote by dim the acl-dimension in a geometric theory, and by $\downarrow$ the corresponding algebraic independence relation (see e.g. [6, Section 2] for details).

Definition 7.1. Let $T$ be an arbitrary theory.

1. An element $a \in \mathbb{M}$ is non-trivial if there exist elements $b, c \in \mathbb{M}$ and a small set $B \subseteq \mathbb{M}$ such that $a \in \operatorname{acl}(b c B) \backslash(\operatorname{acl}(b B) \cup \operatorname{acl}(c B))$.

2. A theory $T$ is non-trivial if there exists a non-trivial element in $\mathbb{M}$; otherwise we call $T$ trivial.

Remark 7.2. It is immediate from the definitions that a geometric theory $T$ is trivial if and only if it has disintegrated algebraic closure - that is, if $\operatorname{acl}(A)=\bigcup_{a \in A} \operatorname{acl}(a)$ for any set $A \subseteq \mathbb{M}$.

Let now $T$ be an arbitrary $\mathcal{L}$-theory with quantifiers elimination (for the questions considered here, we may always assume it replacing $T$ by its Morleyzation) and also eliminating $\exists^{\infty}$. Let $S$ be a distinguished $\mathcal{L}(\emptyset)$-definable set in $T$. We denote by $T_{0, S}$ the theory in a language $\mathcal{L}_{P}:=\mathcal{L} \cup\{P(x)\}$ given by $T \cup\{P(x) \rightarrow S(x)\}$. When working in an $\mathcal{L}_{P}$-structure, we will write $\operatorname{tp}_{\mathcal{L}}$ and acl $\mathrm{L}_{\mathcal{L}}$ to denote the type and the algebraic closure of a tuple in the $\mathcal{L}$-reduct (as opposed to its $\mathcal{L}_{P}$-type $\operatorname{tp}_{\mathcal{L}_{P}}$ and its algebraic closure $\operatorname{acl}_{\mathcal{L}_{P}}$ obtained using all $\mathcal{L}_{P}$-formulas).

\section{Fact 7.3.}

1. [6, Theorem 2.4] The theory $T_{0, S}$ has a model companion $T_{P, S}$ with the following axiomatization: $\mathcal{M} \vDash T_{P, S}$ if and only if

(a) $\mathcal{M} \vDash T$;

(b) For every $\mathcal{L}$-formula $\theta(\bar{x}, \bar{z})$ with $\bar{x}=\left(x_{1}, \ldots, x_{n}\right)$ and every $I \subseteq\{1, \ldots, n\}$, $\mathcal{M}$ satisfies

$$
\begin{gathered}
\forall \bar{z}\left(\left(\left(\exists \bar{x} \theta(\bar{x}, \bar{z}) \wedge\left(\bar{x} \cap \operatorname{acl}_{\mathcal{L}}(\bar{z})=\emptyset\right)\right) \wedge \bigwedge_{i=1}^{n} S\left(x_{i}\right) \wedge \bigwedge_{1 \leq i<j \leq n} x_{i} \neq x_{j}\right)\right. \\
\left.\rightarrow \exists \bar{x}\left(\theta(\bar{x}, \bar{z}) \wedge \bigwedge_{i \in I}\left(x_{i} \in P\right) \wedge \bigwedge_{i \notin I}\left(x_{i} \in S \backslash P\right)\right)\right) .
\end{gathered}
$$

2. Let $\mathcal{M} \models T_{P, S}$. Assume that $\bar{a}, \bar{b}$ are tuples from $M$ and $A \subseteq M$ is a set of parameters. Then the following are equivalent:

(a) $\operatorname{tp}_{\mathcal{L}_{P}}(\bar{a} / A)=\operatorname{tp}_{\mathcal{L}_{P}}(\bar{b} / A)$;

(b) There exists an $A$-isomorphism of $\mathcal{L}_{P}$-structures from $\operatorname{acl}_{\mathcal{L}}(A, \bar{a})$ to $\operatorname{acl}_{\mathcal{L}}(A, \bar{b})$ which carries $\bar{a}$ to $\bar{b}$.

3. If $\mathcal{M} \vDash T_{P, S}, a \in M$ and $A \subseteq M$, then $a \in \operatorname{acl}_{\mathcal{L}}(A) \Longleftrightarrow a \in \operatorname{acl}_{\mathcal{L}_{P}}(A)$. 
One typically refers to $T_{P, S}$ as an expansion of $T$ by a generic predicate on $S$. If the predicate $S$ is equivalent to $x=x$ in $T$, we simply write $T_{P}$ instead of $T_{P, S}$.

Our first aim is to show that if $T$ is a non-trivial geometric theory, then its expansion by a generic predicate is not $n$-dependent for any $n$. In order to produce a definable relation witnessing failure of $n$-dependence for an arbitrary $n$, we consider certain algebraic configurations of 'higher arity'.

Definition 7.4. [5, Definition 2.6] Let $T$ be a geometric theory and $B \subseteq \mathbb{M}$. We say that a tuple $\bar{a}=\left(a_{1}, \ldots, a_{n}\right) \in \mathbb{M}^{n}$ is an algebraic n-gon over $B$ if $\operatorname{dim}(\bar{a} / B)=n-1$, but any subset of $\left\{a_{1}, \ldots, a_{n}\right\}$ of size $n-1$ is independent over $B$.

Note that any tuple obtained by permuting the elements of an algebraic $n$-gon over $B$ is still an algebraic $n$-gon over $B$ (by exchange of acl). Due to the following fact, starting with a non-trivial element, we can find an algebraic $n$-gon for any $n$.

Fact 7.5 [5, Lemma 2.7]. Suppose that $T$ is a geometric theory and $a \in \mathbb{M}$ is non-trivial. Then for every $n \geq 3$, there exist some finite set $B \subseteq \mathbb{M}$ and an algebraic $n$-gon $\left(a_{1}, \ldots, a_{n}\right)$ over $B$ such that $a_{n}=a$.

Proposition 7.6. Assume that $T$ is a geometric theory and there exists a non-trivial element in $S$. Then $T_{P, S}$ is not $n$-dependent for any $n \geq 1$.

In particular, if the algebraic closure in $T$ is not disintegrated, then $T_{P}$ is not n-dependent for any $n \geq 1$.

Proof. Fix $n \geq 1$, and let $\mathbb{M}$ be a monster model of $T_{P, S}$. Note that the $\mathcal{L}$-reduct of $\mathbb{M}$ is a monster model of $T$. By Fact 7.3(3), we have $\operatorname{acl}_{\mathcal{L}_{P}}=\operatorname{acl}_{\mathcal{L}}$ in $\mathbb{M}$, so we will just write acl in the rest of the proof. By assumption, there exists a non-trivial $a \models S(x)$ in $\mathbb{M}$.

By Fact 7.5 , let $a_{1}, \ldots, a_{n+1}, a_{n+2}$ and a finite set $B$ be such that $a_{n+2}=a$ and $\left(a_{1}, \ldots, a_{n+2}\right)$ is an algebraic $(n+2)$-gon over $B$. So in particular, $a_{n+2} \vDash S(x)$. Naming $B$ by constants, without loss of generality, we may assume that $B=\emptyset$. Then $\left\{a_{1}, \ldots, a_{n+1}\right\}$ is an $\downarrow$-independent set. Using extension, symmetry and transitivity, we can choose inductively sequences $\bar{a}_{i}=\left(a_{i}^{j}: j \in \omega\right)$ for $1 \leq i \leq n$ such that:

1. $a_{i}^{j} \downarrow \bar{a}_{<i} a_{i}^{<j} a_{i+1} \ldots a_{n+1}$ for all $1 \leq i \leq n$ and all $j \in \omega$;

2. $a_{i}^{j} \equiv_{\bar{a}_{1} \ldots \bar{a}_{i-1} a_{i+1} \ldots a_{n+1}} a_{i}$ for all $1 \leq i \leq n$ and all $j \in \omega$.

In particular, by basic properties of $\downarrow$ and exchange, (1) implies that

(3) $\left\{a_{i}^{j}: 1 \leq i \leq n, j \in \omega\right\} \cup\left\{a_{n+1}\right\}$ is an $\downarrow$-independent set,

and (2) implies that

(4) $a_{1}^{j_{1}} \ldots a_{n}^{j_{n}} a_{n+1} \equiv a_{1} \ldots a_{n} a_{n+1}$ for all $j_{1}, \ldots, j_{n} \in \omega$.

By assumption, we have $a_{n+2} \in S(\mathbb{M}) \cap \operatorname{acl}\left(a_{1} \ldots a_{n+1}\right)$. Then we can choose a formula $\varphi\left(x_{1}, \ldots, x_{n+2}\right) \in \mathcal{L}$ and $1 \leq k \in \omega$ such that:

(5) $\varphi\left(a_{1}^{\prime}, \ldots, a_{n+1}^{\prime}, x_{n+2}\right) \rightarrow S\left(x_{n+2}\right)$ for any $a_{1}^{\prime}, \ldots, a_{n+1}^{\prime} \in \mathbb{M}$;

(6) $\varphi\left(a_{1}, \ldots, a_{n+1}, x_{n+2}\right)$ isolates $\operatorname{tp}_{\mathcal{L}}\left(a_{n+2} / a_{1} \ldots a_{n+1}\right)$;

(7) $\left|\varphi\left(a_{1}, \ldots, a_{n+1}, \mathbb{M}\right)\right|=k$ and $\left|\varphi\left(a_{1}^{\prime}, \ldots, a_{n+1}^{\prime}, \mathbb{M}\right)\right| \in\{0, k\}$ for any $a_{1}^{\prime}, \ldots, a_{n+1}^{\prime} \in \mathbb{M}$.

Claim 7.7. The following holds:

(8) For any $\left(j_{1}, \ldots, j_{n}\right) \in \omega^{n}$, we have

$$
\left|\varphi\left(a_{1}^{j_{1}}, \ldots, a_{n}^{j_{n}}, a_{n+1}, \mathbb{M}\right)\right|=k \text { and } \varphi\left(a_{1}^{j_{1}}, \ldots, a_{n}^{j_{n}}, a_{n+1}, \mathbb{M}\right) \cap \operatorname{acl}\left(\bar{a}_{1} \ldots \bar{a}_{n}\right)=\emptyset
$$

(9) For any $\left(j_{1}, \ldots, j_{n}\right) \neq\left(j_{1}^{\prime}, \ldots, j_{n}^{\prime}\right) \in \omega^{n}$, we have

$$
\varphi\left(a_{1}^{j_{1}}, \ldots, a_{n}^{j_{n}}, a_{n+1}, \mathbb{M}\right) \cap \varphi\left(a_{1}^{j_{1}^{\prime}}, \ldots, a_{n}^{j_{n}^{\prime}}, a_{n+1}, \mathbb{M}\right)=\emptyset
$$


Proof. Proof of (8). Let $\left(j_{1}, \ldots, j_{n}\right) \in \omega^{n}$ be arbitrary. By the first item in (7), let $\bar{b}$ be the tuple listing all $k$-realizations of $\varphi\left(a_{1}, \ldots, a_{n+1}, x_{n+2}\right)$. By (4), we can choose some tuple $\bar{b}^{\prime}$ in $\mathbb{M}$ such that $a_{1}^{j_{1}} \ldots a_{n}^{j_{n}} a_{n+1} \bar{b}^{\prime} \equiv a_{1} \ldots a_{n} a_{n+1} \bar{b}$. Then each of the $k$ pairwise-distinct elements in $\bar{b}^{\prime}$ is a realization of $\varphi\left(a_{1}^{j_{1}}, \ldots, a_{n}^{j_{n}}, a_{n+1}, x_{n+2}\right)$, and these are all possible realizations by the second item in (7).

Assume now that $b \in \varphi\left(a_{1}^{j_{1}}, \ldots, a_{n}^{j_{n}}, a_{n+1}, \mathbb{M}\right)$ is arbitrary. By (3) and basic properties of algebraic independence, we have $a_{n+1} \downarrow_{a_{1}^{j_{1}} \ldots a_{n}^{j_{n}}} a_{1}^{\neq j_{1}} \ldots a_{n}^{\neq j_{n}}$. Hence if $b \in \operatorname{acl}\left(\bar{a}_{1} \ldots \bar{a}_{n}\right)$, then already $b \in$ $\operatorname{acl}\left(a_{1}^{j_{1}} \ldots a_{n}^{j_{n}}\right)$. But $a_{1}^{j_{1}} \ldots a_{n}^{j_{n}} a_{n+1} b \equiv a_{1} \ldots a_{n} a_{n+1} a_{n+2}$ by (4) and (6), and $a_{n+2} \notin \operatorname{acl}\left(a_{1} \ldots a_{n}\right)$ since $\left(a_{1}, \ldots, a_{n+2}\right)$ is an algebraic $(n+2)$-gon - a contradiction.

Proof of (9) Let $\left(j_{1}, \ldots, j_{n}\right) \neq\left(j_{1}^{\prime}, \ldots, j_{n}^{\prime}\right)$ in $\omega^{n}$ be given, and let $J:=\left\{1 \leq t \leq n: j_{t}=j_{t}^{\prime}\right\}$. Thus $|J|<n$. By (3) and basic properties of algebraic independence, we have

$$
\left(a_{t}^{j_{t}}: t \notin J\right) \perp_{a_{n+1}\left(a_{t}^{j_{t}}: t \in J\right)}\left(a_{t}^{j_{t}^{\prime}}: t \notin J\right)
$$

Using this and the second item in (7), if

$$
b \in \varphi\left(a_{1}^{j_{1}}, \ldots, a_{n}^{j_{n}}, a_{n+1}, \mathbb{M}\right) \cap \varphi\left(a_{1}^{j_{1}^{\prime}}, \ldots, a_{n}^{j_{n}^{\prime}}, a_{n+1}, \mathbb{M}\right),
$$

then

$$
\left.b \in \operatorname{acl}\left(\left(a_{t}^{j_{t}}: t \in J\right) a_{n+1}\right)\right) .
$$

Additionally, by (4) and (6), we have $a_{1}^{j_{1}} \ldots a_{n}^{j_{n}} a_{n+1} b \equiv a_{1} \ldots a_{n} a_{n+1} a_{n+2}$; hence $a_{n+2} \in \operatorname{acl}\left(\left(a_{t}: t \in\right.\right.$ $\left.J) a_{n+1}\right)$. This is a contradiction since $\left(a_{1}, \ldots, a_{n+2}\right)$ is an $(n+2)$-gon and $|J|<n$ and concludes the proof of the claim.

Now, consider the formula

$$
\psi\left(x_{1}, \ldots, x_{n+1}\right):=\exists x_{n+2} \in P \varphi\left(x_{1}, \ldots, x_{n+1}, x_{n+2}\right) .
$$

We will show that $\psi$ is not $n$-dependent. For this, we show that for an arbitrary $m \in \omega, \psi$ shatters $\left(a_{i}^{j}: 1 \leq i \leq n, j \in m\right)$. Toward this, let $I \subseteq m^{n}$ be fixed. Set $\bar{a}:=\left(a_{i}^{j}: 1 \leq i \leq n, 1 \leq j \leq m\right)$, with $a_{i}^{j}$ as chosen in the beginning of the proof, and consider the $\mathcal{L}$-formula

$$
\begin{gathered}
\theta(\bar{x}, \bar{a})=\theta\left(\left(x_{\bar{j}}^{t}: \bar{j} \in m^{n}, 1 \leq t \leq k\right), \bar{a}\right):= \\
\exists x_{n+1} \bigwedge_{\bar{j} \in m^{n}} \bigwedge_{1 \leq t \leq k} \varphi\left(a_{1}^{j_{1}}, \ldots, a_{n}^{j_{n}}, x_{n+1}, x_{\left(j_{1}, \ldots, j_{n}\right)}^{t}\right) \wedge \rho\left(\left(x_{\bar{j}}^{t}: \bar{j} \in m^{n}, 1 \leq t \leq k\right)\right),
\end{gathered}
$$

where $\rho$ is a formula expressing that all of the elements of the tuple $\left(x_{\bar{j}}^{t}: \bar{j} \in m^{n}, 1 \leq t \leq k\right)$ are pairwisedistinct. By the first item in (8), for each $\bar{j} \in m^{n}$, we let $\bar{b}_{\bar{j}}=\left(b_{\bar{j}}^{t}: 1 \leq t \leq k\right)$ be a tuple of length $k$ enumerating the set $\varphi\left(a_{1}^{j_{1}}, \ldots, a_{n}^{j_{n}}, a_{n+1}, \mathbb{M}\right)$ in an arbitrary order, and let $\bar{b}:=\left(b_{\bar{j}}^{t}: \bar{j} \in m^{n}, 1 \leq t \leq k\right)$.

Then we have:

○ $\bar{b} \subseteq S$, by (5).

- All elements of the tuple $\bar{b}$ are pairwise-distinct, by (9).

○ $\bar{b} \cap \operatorname{acl}(\bar{a})=\emptyset$, by the second item in $(8)$.

$\circ \vDash \theta(\bar{b}, \bar{a})$, with $\exists x_{n+1}$ realized by $a_{n+1}$ by the choice of $\bar{b}$.

Hence, applying Fact 7.3, there exists some $\bar{c}=\left(c_{\bar{j}}^{t}: \bar{j} \in m^{n}, 1 \leq t \leq k\right) \subseteq S$ such that 
$\circ \vDash \theta(\bar{c}, \bar{a})$;

○ $\bar{c}_{\bar{j}}:=\left(c_{\bar{j}}^{t}: 1 \leq t \leq k\right) \subseteq P$ for every $\bar{j} \in I$;

○ $\bar{c}_{\bar{j}} \cap P=\emptyset$ for every $\bar{j} \in m^{n} \backslash I$.

As $\models \theta(\bar{c}, \bar{a})$, all elements of $\bar{c}$ are pairwise distinct as it realizes $\rho$, and there exists some $a_{n+1}^{I} \in \mathbb{M}$ such that

$$
\vDash \bigwedge_{\bar{j} \in m^{n}} \bigwedge_{1 \leq t \leq k} \varphi\left(a_{1}^{j_{1}}, \ldots, a_{n}^{j_{n}}, a_{n+1}^{I}, c_{\left(j_{1}, \ldots, j_{n}\right)}^{t}\right) .
$$

In particular, for every $\bar{j}=\left(j_{1}, \ldots, j_{n}\right) \in m^{n}$, every element of the tuple $\bar{c}_{\bar{j}}$ of length $k$ is in the set $\varphi\left(a_{1}^{j_{1}}, \ldots, a_{n}^{j_{n}}, a_{n+1}^{I}, \mathbb{M}\right)$. Hence, by the second item in (7), the tuple $\bar{c}_{\bar{j}}$ lists all of the elements of the set $\varphi\left(a_{1}^{j_{1}}, \ldots, a_{n}^{j_{n}}, a_{n+1}^{I}, \mathbb{M}\right)$. By the choice of $\bar{c}$, it follows that for every $\bar{j} \in m^{n}$,

$$
\vDash \psi\left(a_{1}^{j_{1}}, \ldots, a_{n}^{j_{n}}, a_{n+1}^{I}\right) \Longleftrightarrow i \in I
$$

As $m$ and $I$ were arbitrary, we conclude that $\psi$ is not $n$-dependent by compactness.

Finally, the 'in particular' part of the proposition is immediate by Remark 7.2.

Remark 7.8. The case of $n=1$ of Proposition 7.6 is claimed in [6, Proposition 2.10] without the assumption that $T$ is geometric. However, their proof contains a gap, and the claim is false as witnessed by the following example. Let $T$ be the theory of the infinite branching tree - that is, the theory of an infinite graph $(G, R)$ such that

1. For every vertex $a \in G$, there are infinitely many $b$ such that $a R b$,

2. There are no cycles.

It is not hard to see by back-and-forth that $T$ is complete and admits quantifier elimination after adding distance predicates (which are definable in the graph language using quantifiers). Then $T_{P}$ is stable, for example, since by [26, Theorem 1.4], every expansion of a planar graph by unary predicates is stable. However, acl is not disintegrated (for any $a \in G$ and two elements $b, c$ connected to it, we have that $a \in \operatorname{dcl}(b c)$, but $a \notin \operatorname{acl}(b) \cup \operatorname{acl}(c)$. Note that acl doesn't satisfy exchange in this example since $b \notin \operatorname{acl}(a c)$.

Remark 7.9. In a recent erratum [7] to [6], it is observed that $T_{P, S}$ is not dependent assuming the following stronger variant of the failure of disintegration of acl in $T$ : there exist a small model $M \prec \mathbb{M} \vDash T$ and tuples $a, b$ such that $\operatorname{acl}(M, a, b) \cap S \subsetneq \operatorname{acl}(M, a) \cup \operatorname{acl}(M, b)$, and moreover $\operatorname{tp}(a / M b)$ is finitely satisfiable in $M$.

Next we will show a converse to Proposition 7.6: if the algebraic closure in a geometric theory is disintegrated, then $n$-dependence is preserved after adding a generic predicate. More generally, we consider expansions by 'generic' relations of arity at most $n$.

The following is an analog of Fact 7.3 in this more general setting. It is essentially from [46], though we refer to [32] here. Namely, let $T$ be a complete theory in a language $\mathcal{L}$ with a distinguished $\mathcal{L}(\emptyset)$ definable set $S(x)$, and let $\mathcal{L}^{\prime} \supseteq \mathcal{L}$ be a language such that $\mathcal{L}^{\prime} \backslash \mathcal{L}=\left\{R_{i}: i \in I\right\}$ only contains relational symbols. Then we let $\mathcal{L}_{0}:=\mathcal{L} \cup\left\{S^{*}(x)\right\}$, where $S^{*}(x)$ is a new unary predicate symbol, and let $T_{0}$ be the (complete) $\mathcal{L}_{0}$-theory axiomatized by $T \cup\left\{S(x) \leftrightarrow S^{*}(x)\right\}$. Let $T_{\cap}$ be the reduct of $T_{0}$ to the language $\mathcal{L}_{\cap}:=\left\{S^{*}(x)\right\}$ (so a complete theory of a unary predicate). And for $i \in I$, we let $\mathcal{L}_{i}:=\left\{S^{*}(x)\right\} \cup\left\{R_{i}\right\}$, and let $T_{i}$ be the model companion of the $\mathcal{L}_{i}$-theory $\left\{\forall x_{1} \ldots \forall x_{n} R_{i}\left(x_{1}, \ldots, x_{n}\right) \rightarrow \bigwedge_{1 \leq j \leq n} S^{*}\left(x_{j}\right)\right\}$ (which exists by [46, Theorem 5] or by [6, Remark 2.12.2]). In the next fact, the existence of a model companion $T^{\prime}$ of the theory $T_{\cup}:=\bigcup_{i \in I} T_{i}$ is given by [32, Theorem 5.50], the description of types in $T^{\prime}$

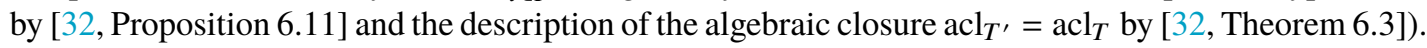


Fact 7.10. Let $T$ be a theory in the language $\mathcal{L}$ eliminating quantifiers and $\exists^{\infty}$, and fix an $\mathcal{L}(\emptyset)$-definable predicate $S$. Let $\mathcal{L}^{\prime} \supseteq \mathcal{L}$ be a language such that $\mathcal{L}^{\prime} \backslash \mathcal{L}$ only contains relational symbols, and consider the $\mathcal{L}^{\prime}$-theory

$$
T_{0}^{\prime}:=T \cup\left\{R\left(x_{1}, \ldots, x_{n}\right) \rightarrow \bigwedge_{1 \leq i \leq n} S\left(x_{i}\right): R \in \mathcal{L}^{\prime} \backslash \mathcal{L}\right\}
$$

Then $T_{0}^{\prime}$ admits a model companion $T^{\prime}$, and working in a monster model of $T^{\prime}$, we have the following: for any tuples $\bar{a}, \bar{b}$ and a subset $A$,

$(\dagger) \operatorname{tp}_{\mathcal{L}^{\prime}}(\bar{a} / A)=\operatorname{tp}_{\mathcal{L}^{\prime}}(\bar{b} / A)$ if and only if there exists an A-isomorphism of $\mathcal{L}^{\prime}$-structures from $\operatorname{acl}_{\mathcal{L}}(A, \bar{a})$ to $\operatorname{acl}_{\mathcal{L}}(A, \bar{b})$ which carries $\bar{a}$ to $\bar{b}$.

We sometimes refer to $T^{\prime}$ as the expansion of $T$ by generic relations in $\mathcal{L}^{\prime} \backslash \mathcal{L}$.

In [25, Lemma 2.1], Hrushovski observes that the random $n$-ary hypergraph is not a finite Boolean combination of relations of arity $n-1$. In order to demonstrate preservation of $n$-dependence in expansions of disintegrated theories by generic $n$-ary relations, we will use the following infinitary generalization of this fact.

Proposition 7.11. For each $n \in \omega, n \geq 1$ and an infinite cardinal $\kappa$, there exists some cardinal $\lambda \geq \kappa$ satisfying the following: let $G_{n, p}^{\prime}$ be a $\lambda$-saturated model of $\operatorname{Th}\left(G_{n, p}\right)$, let $\tilde{\mathcal{L}}$ be an arbitrary relational language with $|\tilde{\mathcal{L}}| \leq \kappa$ containing only relations of arity at most $n-1$, and let $\tilde{O}_{n, p}^{\prime}$ be an expansion of $O_{n, p}^{\prime}$ obtained by adding arbitrary interpretations for all the relations in $\tilde{\mathcal{L}}$. Then the following holds:

there are $g_{i}, h_{i} \in P_{i}^{G_{n, p}^{\prime}}, 1 \leq i \leq n$, such that

$$
\operatorname{qftp}_{\tilde{\mathcal{L}}}\left(g_{1}, \ldots, g_{n}\right)=\operatorname{qftp}_{\tilde{\mathcal{L}}}\left(h_{1}, \ldots, h_{n}\right)
$$

and $G_{n, p}^{\prime} \vDash R_{n}\left(g_{1}, \ldots, g_{n}\right) \wedge \neg R_{n}\left(h_{1}, \ldots, h_{n}\right)$.

Proof. We show this result by induction on $n$ (the base case $n=1$ obviously holds with $\lambda:=\kappa$ ). Now fix $n \geq 2$ and a cardinal $\kappa$. Let $\lambda=\lambda_{n-1}$ satisfy the proposition for $n-1$ and $\kappa$. We will show that $\lambda=\lambda_{n}:=\beth_{n-2}\left(2^{\lambda_{n-1}}\right)^{+}$satisfies the proposition for $n$.

Toward a contradiction, assume that some $\lambda_{n}$-saturated $G_{n, p}^{\prime} \equiv G_{n, p}$, some language $|\tilde{\mathcal{L}}| \leq \kappa$ and some expansion $\tilde{O}_{n, p}^{\prime}$ do not satisfy the proposition - that is, for all $g_{i}, h_{i} \in P_{i}^{G_{n, p}^{\prime}}, 1 \leq i \leq n$,

(*) If $\operatorname{qftp}_{\tilde{\mathcal{L}}}\left(g_{1}, \ldots, g_{n}\right)=\operatorname{qftp}_{\tilde{\mathcal{L}}}\left(h_{1}, \ldots, h_{n}\right) \wedge G_{n, p}^{\prime} \vDash R_{n}\left(g_{1}, \ldots, g_{n}\right)$, then $G_{n, p}^{\prime} \vDash R_{n}\left(h_{1}, \ldots, h_{n}\right)$.

By the choice of $\lambda_{n}$ and Erdôs-Rado, we have $\lambda_{n} \rightarrow\left(\left(2^{\lambda_{n-1}}\right)^{+}\right)_{2^{\lambda_{n-1}}}^{n-1}$; hence we can find some sets $A_{i} \subseteq P_{i}^{G_{n, p}^{\prime}}, 1 \leq i \leq n-1$ such that $\left|A_{i}\right| \geq\left(2^{\lambda_{n-1}}\right)^{+}$and $\operatorname{qftp}_{\tilde{\mathcal{L}}}\left(g_{1}, \ldots, g_{n-1}\right)=\operatorname{qftp}_{\tilde{\mathcal{L}}}\left(h_{1}, \ldots, h_{n-1}\right)$ for all $g_{i}, h_{i} \in A_{i}, 1 \leq i \leq n-1$. Next, we can find a $\lambda_{n-1}$-saturated structure $G_{n-1, p}^{\prime} \equiv G_{n-1, p}$ with $\left|G_{n-1, p}^{\prime}\right| \leq 2^{\lambda_{n-1}}$ and such that $P_{i}^{G_{n-1, p}^{\prime}} \subseteq A_{i}, 1 \leq i \leq n-1$. As $G_{n, p}^{\prime}$ is $\lambda_{n}$-saturated and $\lambda_{n}>2^{\lambda_{n-1}}$, by the axioms of $\operatorname{Th}\left(G_{n, p}\right)$, there exists some $c \in P_{n}^{G_{n, p}^{\prime}}$ such that for all $g_{i} \in P_{i}^{G_{n-1, p}^{\prime}}, 1 \leq i \leq n-1$, we have

$$
G_{n-1, p}^{\prime} \vDash R_{n-1}\left(g_{1}, \ldots, g_{n-1}\right) \Longleftrightarrow G_{n, p}^{\prime} \vDash R_{n}\left(g_{1}, \ldots, g_{n-1}, c\right) .
$$

Without loss of generality, we may assume that all relations in $\tilde{\mathcal{L}}$ are of arity exactly $n-1$. We consider the language $\tilde{\mathcal{L}}_{n-1}$ containing, for each $F \in \tilde{\mathcal{L}}$ and $\Upsilon \in \mathcal{P}(\{1, \ldots, n-1\}) \backslash \emptyset$, an $(n-1-|\Upsilon|)$-ary relational symbol $F_{\Upsilon}$. We define an expansion $\tilde{O}_{n-1, p}^{\prime}$ of $O_{n-1, p}^{\prime}$ in which we interpret each such $F_{\Upsilon} \in \tilde{\mathcal{L}}_{n-1}$

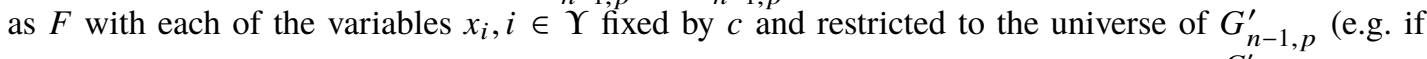
$\Upsilon=\{2, n-1\}$, then $F_{\Upsilon}$ is interpreted as $\left.F\left(x_{1}, c, x_{3}, \ldots, x_{n-2}, c\right) \cap \prod_{i \in\{1,2, \ldots, n-1\} \backslash \Upsilon} P_{i}^{G_{n-1, p}^{\prime}}\right)$. Hence 
$\left|\tilde{\mathcal{L}}_{n-1}\right| \leq \kappa$, and all relations in $\tilde{\mathcal{L}}_{n-1}$ have arity at most $n-2$. Note that by the choice of $A_{i}, 1 \leq i \leq n-1$, we automatically have that for any $F \in \tilde{\mathcal{L}}$ and any $g_{i}, h_{i} \in P_{i}^{G_{n-1, p}^{\prime}}, 1 \leq i \leq n-1$,

$$
\tilde{O}_{n, p}^{\prime} \vDash F\left(g_{1}, \ldots, g_{n-1}\right) \Longleftrightarrow \tilde{O}_{n, p}^{\prime} \vDash F\left(h_{1}, \ldots, h_{n-1}\right) \text {. }
$$

By the choice of the $F_{\Upsilon}$ 's, we have that for any $g_{i}, h_{i} \in P_{i}^{G_{n-1, p}^{\prime}}, 1 \leq i \leq n-1$,

$$
\begin{gathered}
\operatorname{qftp}_{\tilde{\mathcal{L}}_{n-1}}\left(g_{1}, \ldots, g_{n-1}\right)=\operatorname{qftp}_{\tilde{\mathcal{L}}_{n-1}}\left(h_{1}, \ldots, h_{n-1}\right) \Longrightarrow \\
\operatorname{qftp}_{\tilde{\mathcal{L}}}\left(g_{1}, \ldots, g_{n-1}, c\right)=\operatorname{qftp}_{\tilde{\mathcal{L}}}\left(h_{1}, \ldots, h_{n-1}, c\right) .
\end{gathered}
$$

Since $(*)$ holds for $\tilde{O}_{n, p}^{\prime}$, by the choice of $c$ this implies

$$
\begin{gathered}
\operatorname{qftp}_{\tilde{\mathcal{L}}_{n-1}}\left(g_{1}, \ldots, g_{n-1}\right)=\operatorname{qftp}_{\tilde{\mathcal{L}}_{n-1}}\left(h_{1}, \ldots, h_{n-1}\right) \wedge G_{n-1, p}^{\prime} \vDash R_{n-1}\left(g_{1}, \ldots, g_{n-1}\right) \Longrightarrow \\
G_{n-1, p}^{\prime} \vDash R_{n-1}\left(h_{1}, \ldots, h_{n-1}\right) .
\end{gathered}
$$

That is, $\tilde{O}_{n-1, p}^{\prime}$ and $\tilde{\mathcal{L}}_{n-1}$ satisfy $(*)$ for $n-1$ - contradicting the induction hypothesis.

Using Proposition 7.11, we can prove preservation of $n$-dependence.

Proposition 7.12. Let $T$ be a theory in the language $\mathcal{L}$ eliminating $\exists^{\infty}$ (not necessarily geometric), and assume that all elements in $S$ are trivial. Fix $n \geq 1$, and let $T^{\prime}$ be a generic expansion of $T$ in a language $\mathcal{L}^{\prime}$ such that $\mathcal{L}^{\prime} \backslash \mathcal{L}$ only contains relational symbols of arity at most $n$ living on $S$. Then $T^{\prime}$ is $n$-dependent if and only if $T$ is $n$-dependent.

Proof. Assume that the theory $T$ is $n$-dependent, but there is some $\mathcal{L}^{\prime}$-formula $\varphi\left(x ; y_{1}, \ldots, y_{n}\right)$ that is not $n$-dependent in $T^{\prime}$. Let $T^{\mathrm{Sk}}$ be a Skolemization of $T^{\prime}$ with a distinguished constant symbol 0 , in the language $\mathcal{L}^{\mathrm{Sk}} \supseteq \mathcal{L}^{\prime},\left|\mathcal{L}^{\mathrm{Sk}}\right|=\left|\mathcal{L}^{\prime}\right|$. Let $\kappa:=\left|\mathcal{L}^{\prime}\right|$, and let $\lambda$ be as given by Proposition 7.11 for $n$ and $\kappa$.

Let $G_{n, p}^{\prime}$ be a $\lambda$-saturated model of $\operatorname{Th}\left(G_{n, p}\right)$. Working in a monster model $\mathbb{M}$ of $T^{\text {Sk }}$ (which we may assume to be $\left|G_{n, p}^{\prime}\right|^{+}$-saturated in particular), $\varphi\left(x ; y_{1}, \ldots, y_{n}\right)$ is still not $n$-dependent; hence by Proposition 2.8(3), there exist tuples $\left(a_{g}\right)_{g \in G_{n, p}^{\prime}}$ and $b$ such that:

1. $\left(a_{g}\right)_{g \in G_{n, p}^{\prime}}$ is $O_{n, p}^{\prime}$-indiscernible over $\emptyset$ and $G_{n, p}^{\prime}$-indiscernible over $b$, both in the sense of $T^{\mathrm{Sk}}$;

2. $\vDash \varphi\left(b ; a_{g_{1}}, \ldots, a_{g_{n}}\right) \Longleftrightarrow G_{n, p}^{\prime} \vDash R_{n}\left(g_{1}, \ldots, g_{n}\right)$, for all $g_{i} \in P_{i}$.

First we would like to replace each $a_{g}$ and $b$ by their algebraic closures. In order to preserve indiscernibility, we have to enumerate these algebraic closures in a coherent manner, which can be done as follows. Let $\left(f_{\alpha}\left(\bar{y}_{\alpha}\right): \alpha \in \kappa\right)$ be an arbitrary enumeration of all $\mathcal{L}^{\mathrm{Sk}}(\emptyset)$-definable functions. Given an arbitrary tuple $c=\left(c_{j}\right)_{j \in I}$ in $\mathbb{M}$, we let $J_{c}:=\kappa \times I^{<\omega}$ be ordered lexicographically with respect to the ordering on the ordinal $\kappa$ and the ordering on $I$. Then we consider the tuple $\operatorname{Sk}(c)=\left(\operatorname{Sk}_{j}(c): j \in J_{c}\right)$, where for $j=(\alpha, \beta) \in \kappa \times I^{<\omega}, \mathrm{Sk}_{j}(c)$ is $f_{\alpha}\left(\left(c_{t}: t \in \beta\right)\right)$ (or 0 if the sort of the parameter doesn't fit the sort of the variables of the function).

Now for each $1 \leq i \leq n$, we fix an arbitrary $g_{i} \in P_{i}^{G_{n, p}^{\prime}}$. The set of elements appearing in the tuple $\operatorname{Sk}\left(a_{g_{i}}\right)$ is an elementary $\mathcal{L}^{\mathrm{Sk}}$-submodel of $\mathbb{M}$ by Tarski-Vaught. In particular, we can choose some $J_{i} \subseteq J_{a_{g_{i}}}$ so that the tuple $a_{g_{i}}^{\prime}:=\left(\operatorname{Sk}_{j}\left(a_{g_{i}}\right): j \in J_{i}\right)$ enumerates $\operatorname{acl}_{\mathcal{L}}\left(a_{g_{i}}\right)$ without repeated elements. Now for an arbitrary $1 \leq i \leq n$ and $g \in P_{i}^{G_{n, p}^{\prime}}$, we let $a_{g}^{\prime}:=\left(\operatorname{Sk}_{j}\left(a_{g}\right): j \in J_{i}\right)$. Similarly, we choose some $J_{0} \subseteq J_{b}$ so that the tuple $b^{\prime}:=\left(\operatorname{Sk}_{j}(b): j \in J_{0}\right)$ lists $\operatorname{acl}_{\mathcal{L}}(b)$ without repeated elements. Since we have both indiscernibilities in (1) in the sense of $T^{\mathrm{Sk}}$, it follows that:

3. $b^{\prime}$ enumerates $\operatorname{acl}_{\mathcal{L}}(b)$ (by definition);

4. For any $g \in G_{n, p}^{\prime}$, the tuple $a_{g}^{\prime}$ enumerates $\operatorname{acl}_{\mathcal{L}}\left(a_{g}\right)$ without repetitions (as $g \in P_{i}^{G_{n, p}^{\prime}} \Longrightarrow a_{g} \equiv^{\mathcal{L}^{\mathrm{Sk}}}$ $a_{g_{i}}$ by $\left.(1) \Longrightarrow a_{g}^{\prime} \equiv^{\mathcal{L}} a_{g_{i}}^{\prime}\right)$; 
5. $\left|b^{\prime}\right|,\left|a_{g}^{\prime}\right| \leq \kappa$;

6. $\left(a_{g}^{\prime}\right)_{g \in G_{n, p}^{\prime}}$ is $O_{n, p}^{\prime}$-indiscernible over $\emptyset$ and $G_{n, p}^{\prime}$-indiscernible over $b^{\prime}$, both in the sense of $T^{\text {Sk }}$ (and hence in the sense of $T$ as well; again by (1) and definition of $a_{g}^{\prime}$ and $b^{\prime}$ ).

As $T$ is $n$-dependent, it follows from (6) by Proposition 2.9(3) that

7. $\left(a_{g}^{\prime}\right)_{g \in G_{n, p}^{\prime}}$ is $O_{n, p}^{\prime}$-indiscernible over $b^{\prime}$ in the sense of $T$.

Next we consider the $\mathcal{L}^{\prime}$-isomorphism type of the structure induced on the tuple $b^{\prime} a_{g_{1}}^{\prime} \ldots a_{g_{n}}^{\prime}$ as $\bar{g}$ varies, and demonstrate that it cannot reflect exactly the hyper-edge relation $R_{n}$ of $G_{n, p}^{\prime}$. Without loss of generality, we may assume that all relations in $\mathcal{L}^{\prime} \backslash \mathcal{L}$ are of arity exactly $n$. By $O_{n, p}^{\prime}$-indiscernibility in $T^{\prime}$ in (6), for any $F \in \mathcal{L}^{\prime} \backslash \mathcal{L}$, we have

8. $\vDash F\left(a_{g_{1}}, \ldots, a_{g_{n}}\right) \Longleftrightarrow \vDash F\left(a_{h_{1}}, \ldots, a_{h_{n}}\right)$ for all $g_{i}, h_{i} \in P_{i}^{G_{n, p}^{\prime}}, 1 \leq i \leq n$.

We consider an expansion $\tilde{G}_{n, p}^{\prime}$ of $G_{n, p}^{\prime}$ where for each $F\left(x_{1}, \ldots, x_{n}\right) \in \mathcal{L}^{\prime} \backslash \mathcal{L}$, each $1 \leq i \leq n$ and each $j_{t} \in J_{t}, t \in\{0, \ldots, n\} \backslash\{i\}$, we add a new $(n-1)$-ary relation $R_{F, i, \bar{j}} \subseteq \prod_{t \in\{1, \ldots, n\} \backslash\{i\}} P_{t}^{G_{n, p}^{\prime}}$ defined as follows: for any $\left(g_{t}\right)_{t \in\{1, \ldots, n\} \backslash\{i\}} \in \prod_{t \in\{1, \ldots, n\} \backslash\{i\}} P_{t}^{G_{n, p}^{\prime}}$, we have

$$
\left(g_{t}\right)_{t \in\{1, \ldots, n\} \backslash\{i\}} \in R_{F, i, \bar{j}}: \Longleftrightarrow \quad F F\left(a_{g_{1}, j_{1}}^{\prime}, \ldots, a_{g_{i-1}, j_{i-1}}^{\prime}, b_{j_{0}}^{\prime}, a_{g_{i+1}, j_{i+1}}^{\prime}, \ldots, a_{g_{n}, j_{n}}^{\prime}\right),
$$

where $a_{g, j}^{\prime}$ is the $j$ th element of the tuple $a_{g}^{\prime}$ (i.e. $a_{g, j}^{\prime}=\operatorname{Sk}_{j}\left(a_{g}\right)$ ).

Note that taking

$$
\tilde{\mathcal{L}}:=\left\{R_{F, i, \bar{j}}: F \in \mathcal{L}^{\prime} \backslash \mathcal{L}, 1 \leq i \leq n, j_{t} \in J_{t} \text { for } 0 \leq t \leq n\right\} \cup \mathcal{L},
$$

we have $|\tilde{\mathcal{L}}| \leq \kappa$ by (5). Then, by Proposition 7.11 and the choice of $\lambda$, there exist some $g_{i}, h_{i} \in P_{i}^{G_{n, p}^{\prime}}$ for $1 \leq i \leq n$ such that:

9. $\operatorname{qftp}_{\tilde{\mathcal{L}}}\left(g_{1}, \ldots, g_{n}\right)=\operatorname{qftp}_{\tilde{\mathcal{L}}}\left(h_{1}, \ldots, h_{n}\right)$,

10. $G_{n, p}^{\prime} \vDash R_{n}\left(g_{1}, \ldots, g_{n}\right)$,

11. $G_{n, p}^{\prime \prime p} \vDash \neg R_{n}\left(h_{1}, \ldots, h_{n}\right)$.

By (7) $a_{g_{1}}^{\prime} \ldots a_{g_{n}}^{\prime} \equiv_{b^{\prime}}^{\mathcal{L}} a_{h_{1}}^{\prime} \ldots a_{h_{n}}^{\prime}$. Taking any $\mathcal{L}$-automorphism $\sigma$ of $\mathbb{M}$ sending the tuple on the left-hand side to the right-hand side over $b^{\prime}$, we have:

12. $\sigma$ fixes $b^{\prime}$ pointwise, and $\sigma\left(a_{g_{i}}^{\prime}\right)=a_{h_{i}}^{\prime}$ (preserving the ordering of the tuples) for all $1 \leq i \leq n$.

13. $\sigma\left(\operatorname{acl}_{\mathcal{L}}\left(b^{\prime} a_{g_{1}}^{\prime} \ldots a_{g_{n}}^{\prime}\right)\right)=\operatorname{acl}_{\mathcal{L}}\left(b^{\prime} a_{h_{1}}^{\prime} \ldots a_{h_{n}}^{\prime}\right)$ setwise.

14. $\sigma\left(\operatorname{acl}_{\mathcal{L}}\left(b^{\prime} a_{g_{1}}^{\prime} \ldots a_{g_{n}}^{\prime}\right) \cap S\right)=\operatorname{acl}_{\mathcal{L}}\left(b^{\prime} a_{h_{1}}^{\prime} \ldots a_{h_{n}}^{\prime}\right) \cap S$ setwise.

Let now an element $e \in \operatorname{acl}_{\mathcal{L}}\left(b^{\prime} a_{g_{1}}^{\prime} \ldots a_{g_{n}}^{\prime}\right) \cap S$ be arbitrary. As all elements of $S$ are trivial (in $T$ ) by assumption, we have $e \in \operatorname{acl}_{\mathcal{L}}\left(b^{\prime}\right)=b^{\prime}$ or $e \in \operatorname{acl}_{\mathcal{L}}\left(a_{g_{i}}^{\prime}\right)=a_{g_{i}}^{\prime}$ for some $1 \leq i \leq n$. Hence $\operatorname{acl}_{\mathcal{L}}\left(b^{\prime} a_{g_{1}}^{\prime} \ldots a_{g_{n}}^{\prime}\right) \cap S \subseteq b^{\prime} a_{g_{1}}^{\prime} \ldots a_{g_{n}}^{\prime}$, and so also $\operatorname{acl}_{\mathcal{L}}\left(b^{\prime} a_{h_{1}}^{\prime} \ldots a_{h_{n}}^{\prime}\right) \cap S \subseteq b^{\prime} a_{h_{1}}^{\prime} \ldots a_{h_{n}}^{\prime}$. Given any tuple $c$ of elements from acl $\operatorname{~}_{\mathcal{L}}\left(b^{\prime} a_{g_{1}}^{\prime} \ldots a_{g_{n}}^{\prime}\right)$, by definition of $T^{\prime}$ it can satisfy a relation $F \in \mathcal{L}^{\prime} \backslash \mathcal{L}$ only if $c$ is entirely contained in $S$, hence only if $c$ is contained in the tuple $b^{\prime} a_{g_{1}}^{\prime} \ldots a_{g_{n}}^{\prime}$. But by (8) and (9) (unwinding the definition of $\tilde{\mathcal{L}}$ and using (12)), for any tuple $c$ in $b^{\prime} a_{g_{1}}^{\prime} \ldots a_{g_{n}}^{\prime}$ and $F \in \mathcal{L}^{\prime} \backslash \mathcal{L}$, $c$ satisfies $F$ if and only if $\sigma(c)$ satisfies $F$. And of course $c$ and $\sigma(c)$ agree on all $\mathcal{L}$-formulas. We

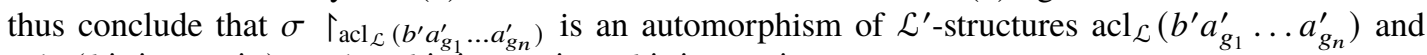
$\operatorname{acl}_{\mathcal{L}}\left(b^{\prime} a_{h_{1}}^{\prime} \ldots a_{h_{n}}^{\prime}\right)$ sending $b^{\prime} a_{g_{1}}^{\prime} \ldots a_{g_{n}}^{\prime}$ to $b^{\prime} a_{h_{1}}^{\prime} \ldots a_{h_{n}}^{\prime}$. 
Hence by Fact $7.10(\dagger)$, we have

$$
\operatorname{tp}_{\mathcal{L}^{\prime}}\left(a_{g_{1}} \ldots a_{g_{n}} / b\right)=\operatorname{tp}_{\mathcal{L}^{\prime}}\left(a_{h_{1}} \ldots a_{h_{n}} / b\right) .
$$

But this contradicts the choice of $\varphi$ in view of (10) and (11).

Finally, the converse implication of the proposition is obvious.

Combining Propositions 7.6 and 7.12, we thus have the following 'baby case' of the relationship of the collapse of $n$-dependence to dependence and complicated geometry of algebraic closure that we expect to happen for fields.

Corollary 7.13. Let $T$ be a geometric theory. The following are equivalent:

1. $T_{P}$ is dependent.

2. $T_{P}$ is $n$-dependent for some $n \in \omega$.

3. $T$ has disintegrated algebraic closure.

Problem 7.14. It should be possible to generalize these results on preservation of $n$-dependence to interpolative fusions of theories as studied in [32].

\section{Appendix A. An explicit isomorphism in Kaplan-Scanlon-Wagner, by Martin Bays}

Let $k$ be a perfect field of characteristic $p>0$. Let $\phi$ be the Frobenius automorphism, $\phi(x):=x^{p}$, and let $\wp$ be the Artin-Schreier map, $\wp(x):=\phi(x)-x=x^{p}-x$. Let $\bar{a}=\left(a_{0}, \ldots, a_{m}\right) \in k^{m+1}$. Let $G_{\bar{a}}:=\left\{\bar{x} \mid a_{0} \wp\left(x_{0}\right)=\ldots=a_{m} \wp\left(x_{m}\right)\right\}$, considered as an algebraic subgroup over $k$ of the Cartesian power of the additive group $\mathbb{G}_{a}^{m+1}$. A crucial step in the proof in [31] of Artin-Schreier closedness of dependent fields is to show that if $\bar{a}$ is an algebraically independent tuple - that is, $\operatorname{trd}\left(\mathbb{F}_{p}(\bar{a}) / \mathbb{F}_{p}\right)=m+1-$ then $G_{\bar{a}}$ is isomorphic over $k$ to the additive group, as algebraic groups. Hempel [22] improves this by showing that the same holds when the assumption is weakened to $\mathbb{F}_{p^{-}}$ linear independence of $\left(a_{0}^{-1}, \ldots, a_{m}^{-1}\right)$. In both cases, the proof is rather indirect, going via showing that $G_{\bar{a}}$ is connected and then referring to some standard theorems characterising vector groups in positive characteristic. The purpose of this appendix is to exhibit such an isomorphism. Thanks to Mohammed Bardestani and Pierre Touchard for helpful discussion.

First we need the following fact about the Moore matrix, being the analogue of the Wronskian matrix with Frobenius in place of differentiation. For completeness, we include a proof.

Fact A.1. Let $k$ be a perfect field of characteristic $p>0$. Let $\bar{c}=\left(c_{0}, \ldots, c_{m}\right) \in k^{m+1}$. Then the Moore matrix $M:=\left(\phi^{i}\left(c_{j}\right)\right)_{0 \leq i, j \leq m}$ is singular if and only if $\bar{c}$ is $\mathbb{F}_{p}$-linearly dependent.

Proof. Suppose $\bar{c}$ is $\mathbb{F}_{p}$-linearly dependent, say $\sum_{j=0}^{m} \lambda_{j} c_{j}=0$ with $\bar{\lambda} \in \mathbb{F}_{p}^{m+1} \backslash\{\overline{0}\}$. Then $\sum_{j=0}^{m} \lambda_{j} \phi^{i}\left(c_{j}\right)=0$ for all $i$, so $M$ is singular.

The converse is clear for $m=0$. So suppose $m \geq 1$, and $\bar{c}$ is $\mathbb{F}_{p}$-linearly independent but $M$ is singular, say $\bigwedge_{0 \leq i \leq m} \sum_{j \geq 0} \mu_{j} \phi^{i}\left(c_{j}\right)=0$ with $\bar{\mu} \in k \backslash\{\overline{0}\}$. Since $\left(c_{1}, \ldots, c_{m}\right)$ is $\mathbb{F}_{p}$-linearly independent, we may inductively assume that the $m \times m$ matrix $M^{\prime}:=\left(\phi^{i}\left(c_{j}\right)\right)_{0<i, j \leq m}$ is non-singular. It follows that $\mu_{0} \neq 0$. Now, let $\alpha:=\frac{\phi\left(\mu_{0}\right)}{\mu_{0}} \neq 0$. Then for $0<i \leq m$, we have

$$
\begin{aligned}
\phi\left(\mu_{0}\right) \phi^{i}\left(c_{0}\right)+\sum_{j \geq 1} \phi\left(\mu_{j}\right) \phi^{i}\left(c_{j}\right) & =\sum_{j \geq 0} \phi\left(\mu_{j}\right) \phi^{i}\left(c_{j}\right) \\
& =\phi\left(\sum_{j \geq 0} \mu_{j} \phi^{i-1}\left(c_{j}\right)\right) \\
& =0
\end{aligned}
$$




$$
\begin{aligned}
& =\alpha \cdot \sum_{j \geq 0} \mu_{j} \phi^{i}\left(c_{j}\right) \\
& =\phi\left(\mu_{0}\right) \phi^{i}\left(c_{0}\right)+\sum_{j \geq 1} \alpha \mu_{j} \phi^{i}\left(c_{j}\right) .
\end{aligned}
$$

Consequently $\sum_{j \geq 1} \phi\left(\mu_{j}\right) \phi^{i}\left(c_{j}\right)=\sum_{j \geq 1} \alpha \mu_{j} \phi^{i}\left(c_{j}\right)$. By non-singularity of $M^{\prime}$, we deduce that $\bigwedge_{0 \leq j \leq m} \phi\left(\mu_{j}\right)=\alpha \mu_{j}$. Let $\beta$ (in an extension of $k$ ) be such that $(\beta)^{p-1}=\alpha$ and set $\lambda_{j}:=\frac{\mu_{j}}{\beta}$. Then either $\lambda_{j}=\mu_{j}=0$ or

$$
\lambda_{j}^{p-1}=\frac{\mu_{j}^{p-1}}{\beta^{p-1}}=\frac{\phi\left(\mu_{j}\right)}{\alpha \mu_{j}}=1 .
$$

Hence $\bar{\lambda}_{j} \in \mathbb{F}_{p}^{m+1} \backslash\{\overline{0}\}$. But $\sum_{j \geq 0} \lambda_{j} c_{j}=\frac{1}{\beta} \sum_{j \geq 0} \mu_{j} c_{j}=0$, contradicting $\mathbb{F}_{p}$-linear independence of $\bar{c}$.

Now let $\bar{a}=\left(a_{0}, \ldots, a_{m}\right) \in k^{m+1}$, and suppose $\bar{b}:=\left(a_{0}^{-1}, \ldots, a_{m}^{-1}\right)$ is $\mathbb{F}_{p}$-linearly independent.

Write $\delta_{i, j}$ for the Kronecker delta. By Fact A.1 applied to $\phi^{-m}(\bar{b}),\left(\phi^{-i}\left(b_{j}\right)\right)_{0 \leq i, j \leq m}$ is nonsingular. Since $k$ is perfect, $\phi^{-i}\left(b_{j}\right) \in k$. So there exists $\bar{\alpha}=\left(\alpha_{0}, \ldots, \alpha_{m}\right) \in k^{m+1} \backslash\{\overline{0}\}$ such that $\bigwedge_{0 \leq i \leq m} \sum_{j \geq 0} \phi^{-i}\left(b_{j}\right) \alpha_{j}=\delta_{0, i}$.

Claim A.2. $\bar{\alpha}$ is $\mathbb{F}_{p}$-linearly independent.

Proof. Suppose not, so (permuting if necessary) we have $\alpha_{0}=\sum_{j \geq 1} \lambda_{j} \alpha_{j}$ with $\lambda_{j} \in \mathbb{F}_{p}$. Then for $1 \leq i \leq m$, we have $\phi^{-i}\left(b_{0}\right) \sum_{j \geq 1} \lambda_{j} \alpha_{j}+\sum_{j \geq 1} \phi^{-i}\left(b_{j}\right) \alpha_{j}=\delta_{0, i}=0$, and so $\sum_{j \geq 1} \alpha_{j} \phi^{-i}\left(b_{j}+\lambda_{j} b_{0}\right)=0$.

But $\alpha_{j} \neq 0$ for some $j \geq 1$, since $\bar{\alpha} \neq \overline{0}$. So by Fact A.1, $\left(b_{j}+\lambda_{j} b_{0}\right)_{j \geq 1}$ is $\mathbb{F}_{p}$-linearly dependent, and consequently so is $\bar{b}$, contrary to assumption.

We proceed to define an algebraic isomorphism over $k$ of $G_{\bar{a}}$ with the additive group. So let $K \geq k$ be an arbitrary field extension, and let $\bar{x} \in G_{\bar{a}}(K)$ : that is, $a_{0} \wp\left(x_{0}\right)=\ldots=a_{m} \wp\left(x_{m}\right)$. Set

$$
t:=\sum_{j \geq 0} \alpha_{j} x_{j}
$$

Claim A.3. For $i \geq 0$, we have $\phi^{i}(t)=\sum_{j \geq 0} \phi^{i}\left(\alpha_{j}\right) x_{j}$.

Proof. This holds by definition for $i=0$. For $i>0$, we have

$$
\sum_{j \geq 0} \frac{\phi^{i}\left(\alpha_{j}\right)}{a_{j}}=\phi^{i}\left(\sum_{j \geq 0} \phi^{-i}\left(b_{j}\right) \alpha_{j}\right)=\phi^{i}\left(\delta_{0, i}\right)=0 .
$$

Using this, induction and the equations of $G_{\bar{a}}$, we find

$$
\begin{aligned}
\phi^{i}(t) & =\phi\left(\sum_{j \geq 0} \phi^{i-1}\left(\alpha_{j}\right) x_{j}\right) \\
& =\sum_{j \geq 0} \phi^{i}\left(\alpha_{j}\right) \phi\left(x_{j}\right) \\
& =\sum_{j \geq 0} \phi^{i}\left(\alpha_{j}\right)\left(\wp\left(x_{j}\right)+x_{j}\right)
\end{aligned}
$$




$$
\begin{aligned}
& =\sum_{j \geq 0} \frac{\phi^{i}\left(\alpha_{j}\right)}{a_{j}} a_{j} \wp\left(x_{j}\right)+\sum_{j \geq 0} \phi^{i}\left(\alpha_{j}\right) x_{j} \\
& =\sum_{j \geq 0} \phi^{i}\left(\alpha_{j}\right) x_{j} .
\end{aligned}
$$

Now by Claim A.2 and Fact A.1, the matrix $\left(\phi^{i}\left(\alpha_{j}\right)\right)_{0 \leq i, j \leq m}$ is non-singular, so say $\left(\beta_{i j}\right)_{0 \leq i, j \leq m}$ is the inverse, where $\beta_{i j} \in k$.

Then by Claim A.3, $x_{i}=\sum_{j \geq 0} \beta_{i j} \phi^{j}(t)$.

So we have defined an isomorphism over $k$ of affine varieties

$$
\begin{array}{rlr}
G_{\bar{a}} & \rightarrow & \mathbb{G}_{a} \\
\bar{x} & \mapsto \sum_{j \geq 0} \alpha_{j} x_{j} \\
\left(\sum_{j \geq 0} \beta_{i j} \phi^{j}(t)\right)_{i} & \longleftrightarrow & t
\end{array}
$$

between $G_{\bar{a}}$ and the additive group $\mathbb{G}_{a}$; since the polynomials involved are additive polynomials, this is an isomorphism of algebraic groups.

Acknowledgements. We are grateful to the referee for many valuable suggestions on improving the paper. We thank Martin Bays for agreeing to include his result as an appendix. We thank Itay Kaplan for helpful discussions concerning Section 4.2; Nick Ramsey for suggesting the question considered in Section 6 and for a helpful discussion concerning Section 5; Kota Takeuchi for a discussion concerning Section 5.1; and Erik Walsberg for a discussion concerning Section 7.

Financial support. Chernikov and Hempel were partially supported by the NSF Research Grant DMS-1600796 and by the NSF CAREER grant DMS-1651321. Bays was supported in part by the Deutsche Forschungsgemeinschaft (DFG, German Research Foundation) under Germany's Excellence Strategy EXC 2044-390685587, Mathematics Münster: Dynamics-GeometryStructure.

Conflict of Interest: None.

\section{References}

[1] Vladimir Igorevich Arnol'd. On functions of three variables. In Doklady Akademii Nauk, volume 114, pages 679-681. Russian Academy of Sciences, 1957.

[2] John T Baldwin and Jan Saxl. Logical stability in group theory. Journal of the Australian Mathematical Society, 21(3): 267-276, 1976.

[3] Andreas Baudisch. Mekler's construction preserves CM-triviality. Annals of Pure and Applied Logic, 115(1-3): 115-173, 2002.

[4] Andreas Baudisch. Neostability-properties of Fraïssé limits of 2-nilpotent groups of exponent $p>2$. Archive for Mathematical Logic, 55(3-4): 397-403, 2016.

[5] Alexander Berenstein and Evgueni Vassiliev. Geometric structures with a dense independent subset. Selecta Mathematica, 22(1): 191-225, 2016.

[6] Zoé Chatzidakis and Anand Pillay. Generic structures and simple theories. Annals of Pure and Applied Logic, 95(1-3): 71-92, 1998.

[7] Zoé Chatzidakis and Anand Pillay. Errata of the paper "Generic structures and simple theories", Annals of Pure and Applied Logic 95 (1998) 71 - 92. https://www.math.ens.fr/ zchatzid/papiers/errtpta.pdf, 2020.

[8] Gregory Cherlin and Ehud Hrushovski. Finite structures with few types, volume 152 of Annals of Mathematics Studies. Princeton University Press, Princeton, NJ, 2003.

[9] Gregory Cherlin and Saharon Shelah. Superstable fields and groups. Annals of mathematical logic, 18(3): 227-270, 1980.

[10] Artem Chernikov. Lecture notes on stability theory. AMS Open Math Notes (OMN:201905.110792), 2019.

[11] Artem Chernikov and Nadja Hempel. Mekler's construction and generalized stability. Israel Journal of Mathematics, 230(2): 745-769, 2019.

[12] Artem Chernikov and Nadja Hempel. On $n$-dependent groups and fields III. In preparation, 2021+.

[13] Artem Chernikov, Itay Kaplan, and Saharon Shelah. On non-forking spectra. Journal of the European Mathematical Society, 18(12): 2821-2848, 2016.

[14] Artem Chernikov, Daniel Palacin, and Kota Takeuchi. On n-dependence. Notre Dame J. Form. Log., 60(2): 195-214, 2019. 
[15] Artem Chernikov and Nicholas Ramsey. On model-theoretic tree properties. Journal of Mathematical Logic, 16(02):1650009, 2016.

[16] Artem Chernikov and Saharon Shelah. On the number of Dedekind cuts and two-cardinal models of dependent theories. Journal of the Institute of Mathematics of Jussieu, 15(4): 771-784, 2016.

[17] Artem Chernikov and Henry Towsner. Hypergraph regularity and higher arity VC-dimension. Preprint, arXiv:2010.00726, 2020.

[18] Jan Dobrowolski. Sets, groups, and fields definable in vector spaces with a bilinear form. Preprint, arXiv:2004.07238, 2020.

[19] Jean-Louis Duret. Les corps faiblement algébriquement clos non séparablement clos ont la propriété d'indépendance. In Model theory of algebra and arithmetic, pages 136-162. Springer, 1980.

[20] Jakub Gismatullin. Model theoretic connected components of groups. Israel J. Math., 184: 251-274, 2011.

[21] Nicolas Mark Granger. Stability, simplicity and the model theory of bilinear forms. $\mathrm{PhD}$ thesis, University of Manchester, 1999.

[22] Nadja Hempel. On $n$-dependent groups and fields. Mathematical Logic Quarterly, 62(3): 215-224, 2016.

[23] Ehud Hrushovski. Unidimensional theories are superstable. Annals of Pure and Applied Logic, 50(2): 117-137, 1990.

[24] Ehud Hrushovski. The Mordell-Lang conjecture for function fields. Journal of the American mathematical society, 9(3): 667-690, 1996.

[25] Ehud Hrushovski. Pseudo-finite fields and related structures. In Model theory and applications, volume 11 of Quad. Mat., pages 151-212. Aracne, Rome, 2002.

[26] A Ivanov. The structure of superflat graphs. Fundamenta Mathematicae, 143(2): 107-117, 1993.

[27] Will Johnson. Dp-finite fields I (A): The infinitesimals. Annals of Pure and Applied Logic, page 102947, 2021.

[28] Will Johnson. Dp-finite fields I (B): Positive characteristic. Annals of Pure and Applied Logic, page 102949, 2021.

[29] Will Johnson, Chieu-Minh Tran, Erik Walsberg, and Jinhe Ye. Étale-open topology and the stable field conjecture. Preprint, arXiv:2009.02319, 2020.

[30] William Andrew Johnson. Fun with Fields. ProQuest LLC, Ann Arbor, MI, 2016. PhD thesis, University of California, Berkeley.

[31] Itay Kaplan, Thomas Scanlon, and Frank O Wagner. Artin-Schreier extensions in NIP and simple fields. Israel Journal of Mathematics, 185(1): 141-153, 2011.

[32] Alex Kruckman, Minh Chieu Tran, and Erik Walsberg. Interpolative fusions. Preprint, arXiv:1811.06108, 2018.

[33] Angus Macintyre. On $\omega_{1}$-categorical theories of fields. Fundamenta Mathematicae, 1(71): 1-25, 1971.

[34] David Marker. Model theory: An introduction, volume 217 of Graduate Texts in Mathematics. Springer-Verlag, New York, 2002.

[35] William Mitchell. Aronszajn trees and the independence of the transfer property. Annals of Mathematical Logic, 5(1): 21-46, 1972.

[36] A. Pillay, T. Scanlon, and F. O. Wagner. Supersimple fields and division rings. Math. Res. Lett., 5(4): 473-483, 1998.

[37] Klaus-Peter Podewski. Minimale ringe. Math. Phys. Semesterberichte, 22:193-197, 1973.

[38] S. Shelah. Classification theory and the number of nonisomorphic models, volume 92 of Studies in Logic and the Foundations of Mathematics. North-Holland Publishing Co., Amsterdam, second edition, 1990.

[39] Saharon Shelah. Minimal bounded index subgroup for dependent theories. Proceedings of the American Mathematical Society, 136(3): 1087-1091, 2008.

[40] Saharon Shelah. Strongly dependent theories. Israel Journal of Mathematics, 204(1): 1-83, 2014.

[41] Saharon Shelah. Definable groups for dependent and 2-dependent theories. Sarajevo J. Math., 13(25)(1): 3-25, 2017.

[42] Pierre Simon. A guide to NIP theories, volume 44 of Lecture Notes in Logic. Association for Symbolic Logic, Chicago, IL; Cambridge Scientific Publishers, Cambridge, 2015.

[43] Katrin Tent and Martin Ziegler. A course in model theory, volume $\mathbf{4 0}$ of Lecture Notes in Logic. Association for Symbolic Logic, La Jolla, CA; Cambridge University Press, Cambridge, 2012.

[44] Caroline Terry. $\mathrm{VC}_{\ell}$-dimension and the jump to the fastest speed of a hereditary $\mathcal{L}$-property. Proceedings of the American Mathematical Society, 146(7): 3111-3126, 2018.

[45] Laurentius Petrus Dignus van den Dries. Model Theory of Fields: Decidability and Bounds for Polynomial Ideals. PhD thesis, Rijksuniversiteit Utrecht, 1978.

[46] Peter M. Winkler. Model-completeness and Skolem expansions. In Model theory and algebra (memorial tribute to Abraham Robinson), pages 408-463. Lecture Notes in Math., Vol. 498. 1975.

[47] Boris Zilber. Uncountably categorical theories, volume 117 of Translations of Mathematical Monographs. American Mathematical Society, Providence, RI, 1993. Translated from the Russian by D. Louvish. 